\title{
15. X-RAY MINERALOGY STUDIES, LEG 4
}

\author{
R. W. Rex and B. Murray, University of California, \\ Riverside, California
}

\begin{abstract}
METHOD
The analytical details of the X-ray mineralogy analyses are discussed in Appendix III of this volume. The data presented here involve X-ray diffraction analyses of bulk samples of the sediment as collected, washed to remove sea salt, and then ground to less than 15-micron size. A calcite-free sample was prepared by dissolution of calcite by buffered acetic acid and then sized to separate the 2 to 20 -micron fraction. This fraction is composited, and the composite intervals are shown in the logs in this section of the report. The 2 to 20 micron fraction is particularly informative showing variations in minor constituents that appear in the smear slides inspected under the microscope. In general the bulk sample X-ray analyses differ from the smear slide analyses in missing many of the minor constituents which are evident both in the 2 to 20 -micron fractions as seen by X-ray analysis and in the microscope smear slides. The bulk analysis, however, is a better quantitative indication of the relative abundances of the major constituents in the sediment samples.
\end{abstract}

\section{ANALYTICAL RESULTS}

\section{Site 23}

This site, near the foot of the continental rise, contains a relatively uniform mixture of turbidite type material derived from continental sources and a few volcanic ash ridge zones that have altered to montmorillonite and, in a few cases, to zeolite (Figure 1). The principal variations are between the abundance of calcite of biological origin and the detrital assemblage of quartz, kaolinite, mica, potassium feldspar, and plagioclasefeldspar. The 2 to 20-micron fraction contains notable amounts of rhodochrosite only in the Pleistocene portion of the section (Figure 2). Siderite on the other hand occurs in the Pleistocene as well as the Miocene sediments as a minor accessory carbonate mineral. Dolomite occurs in all the composite samples from the Lower Miocene to the Pleistocene. Abundant phillipsite in the 2 to 20-micron fraction and associated volcanic glass extensively altered to montmorillonite are found in great abundance in Core 4 and the uppermost part of Core 5. The abundant phillipsite in 2 to 20 micron fraction is not evident in the bulk samples. Considerable quantities of pyrite occur in the 2 to 20 micron fraction at a depth of approximately 121 meters in the Lower Miocene section. The quartz-plagioclase covariance, which the authors consider to be typical of the oceanic red clay facies, is not observed in the sediment of Site 23, suggesting that this sediment is predominantly of a near continental origin.

\section{Site 24}

Site 24 is only about ten miles from Site 23 and shows a grossly similar mineralogical composition to Site 23 . The sediment assemblage has the gross characteristics of a continental turbidite assemblage (Figure 3). The 2 to 20-micron calcite-free fraction is very similar to that observed in Site 23, except no rhodochrosite is found in this locality (Figure 4). A second offset hole entitled Hole 24A cored a deeper interval than encountered in Hole 24. Hole 24A covers the Middle Eocene and Upper Paleocene section. The sediments are characterized by the predominant occurrence of cristobalite with lesser quantities of quartz, mica, K-feldspar, plagioclase, and occasional pyrite (Figure 5). The 2 to 20 -micron fraction contained a minor amount of barite in the Upper Paleocene section. Pyrite is found in all the composite samples of the 2 to 20-micron fraction of Hole 24A (Figure 6). Montmorillonite trapped within ash fragment particle also constitutes a major portion of the 2 to 20 -micron fraction.

\section{Site 25}

This site is predominantly calcite-rich foraminiferal ooze and related calcareous sediments, with very small amounts of quartz and kaolin in the bulk samples (Figures 7 and 8 ). The 2 to 20 -micron fractions show some dolomite in the Upper Miocene and relatively common quartz, kaolin, mica, K-feldspar and plagioclase-feldspar throughout most of the section ranging from the Pleistocene to the Upper Miocene (Figures 9 and 10).

\section{Site 26}

This hole, located near the Vema Fracture Zone, contains sediments of Quaternary age. Quartz is the most common mineral ranging up to 61 per cent of the bulk sediments with variable abundant amounts of mica and kaolin, lesser amounts of chlorite, K-feldspar, and plagioclase-feldspar (Figures 11 and 12). The only constituent to vary significantly in this assemblage is the $\mathrm{K}$-feldspar, which is missing in the lower portion of the section. The mineral assemblage is typical of the different size fractions associated with turbidites that occur in a trough in the Vema Fracture Zone area. Chlorite is present in appreciable concentrations in this area, 
whereas it is missing in some of the older sediments in the South Atlantic. This appears to be primarily a particle size effect because the sediments in Site 26 are frequently very coarse.

\section{Site 27}

These cores, in the western part of the Atlantic Basin about 250 miles east of the Lesser Antilles, are characterized by a predominant alternation between the calcitic fossiliferous zones and quartz-rich clastic zones. An unusually high concentration of siderite is found in the Oligocene which is anomalous for most of the rest of the stratigraphic section. Clinoptilolite, zeolite, and abundant montmorillonite are characteristic of an ashy zone that separates the Upper Eocene from the Oligocene (Figures 13 and 14). Pyrite is found occasionally, and chlorite occurs throughout the section ranging from Upper Eocene to Pliocene, suggesting that the major provenance in this area has remained unchanged throughout most of Tertiary time. There is sufficient altered volcanic glass throughout the section that the 2 to 20 -micron fraction contains abundant montmorillonite trapped in the altered ash fragment (Figures 15 and 16).

\section{Site 28}

This area is characterized by a somewhat unusual lithology with abundant clinoptilolite, chlorite, montmorillonite, as well as calcite in the Middle Eocene overlain by a section of uncertain age that contains a very high concentration of plagioclase, chlorite, and possibly the ordered mixed layer chlorite-montmorillonite called corrensite (Figure 17). This mixed layer clay is usually thought to be indicative of low temperature alteration. The 2 to 20 -micron fraction shows the same minerals evident in the bulk analysis plus appreciable quantities of cristobalite in the Middle Eocene section as well as small quantities of siderite (Figure 18).

\section{Site 29}

This core drilled in the central Venezuelan Basin recovered an extensive section ranging from the Recent to the mid-Eocene. The Recent, Pleistocene, Pliocene, and Miocene is characterized by abundant calcite, lesser quantities of quartz and appreciable quantities of kaolin, mica, plagioclase, and chlorite (Figure 19). A zone of clinoptilolite occurrence is found in the Lower Miocene. The mid-Eocene is distinctive in its alteration between predominantly quartz and calcite. Here quartz appears to be the result of recrystallization of silica, and the cherts contain no cristobalite but occur entirely as quartz cherts. Occasional moderate quantities of montmorillonite are interspersed between the cherty zones and are found where calcite is abundant. Extensive quantities of sepiolite are found in a portion of the mid-Eocene section. The 2 to 20 -micron fraction contains modest amounts of detrital kaolin, mica, and
K-feldspar as well as small amounts of quartz in this particular size range (Figure 20). The chert, on the other hand, is much coarser than 20 microns. Hole 29A appears to be similar to the upper portion of Hole 29 (Figures 21 and 22). Hole 29B again is similar to Hole 19 , except that much larger quantities of dolomite are found in the sepiolite-containing zone in the Middle Eocene, and a predominantly dolomitic zone occurs in Core 10 (Figures 23 and 24). Hole 29B has higher abundances of plagioclase then seen in Hole 29, and there is much more montmorillonite in the Upper Eocene and Middle Eocene of Hole 29B than found in 29. The cherts in Site 29 all appear to have been converted to quartz and are not present as cristobalite.

\section{Site 30}

The core was drilled on Site 30 in an area expected to have more calcium carboante and to provide an extensive stratigraphic section. The mineralogy of the sediments encountered consisted primarily of calcite and quartz with a typical detrital assemblage of mica, plagioclase, kaolinite, chlorite, associated with the quartz abundances. Occasional pyrite and barite, as well as several siderite zones, were encountered in the Upper Tertiary. Abundant clinoptilolite was found in the Middle Miocene. K-feldspars are abundant in the Miocene, but are not in the Pleistocene (Figure 25). The 2 to 20micron fraction contains scattered but abundant pyrite throughout the cored intervals, and a hematite sample was found at or near the break between the Pleistocene and the Middle Miocene (Figure 26).

\section{Site 31}

The sediments at this site consisted almost entirely of calcite with lesser quantities of quartz, kaolin, mica, plagioclase, chlorite and occasional montmorilloniterich zones. Clinoptilolite occurred in abundance in a zone in the Lower Miocene (Figure 27). Scattered pyrite is found in the 2 to 20 -micron fraction (Figure 28).

\section{SUMMARY}

The mineralogy of the sediments from Leg 4 is characteristically both diverse and uniform. The diversities are associated primarily with the alternation of the continental turbidite assemblages; with the marine pyroclastic alteration products, such as zeolites, montmorillonite, etc. To this assemblage should be added the diagenetic alteration products, such as, secondary carbonates including dolomite, siderite, and rhodochrosite. Both cristobalite and quartz cherts are found, and in the Venezuelan Basin a substantial sepiolite occurrence is evident. The Venezuela sepiolite is approximately of equivalent age to the gypsum evaporite found in the northern basin of Trinidad and on the islands of Jamaica and Hispanola. There appears to be a general time correspondence between the abundant quartz chert sepiolite and evaporite deposits in the Caribbean. 


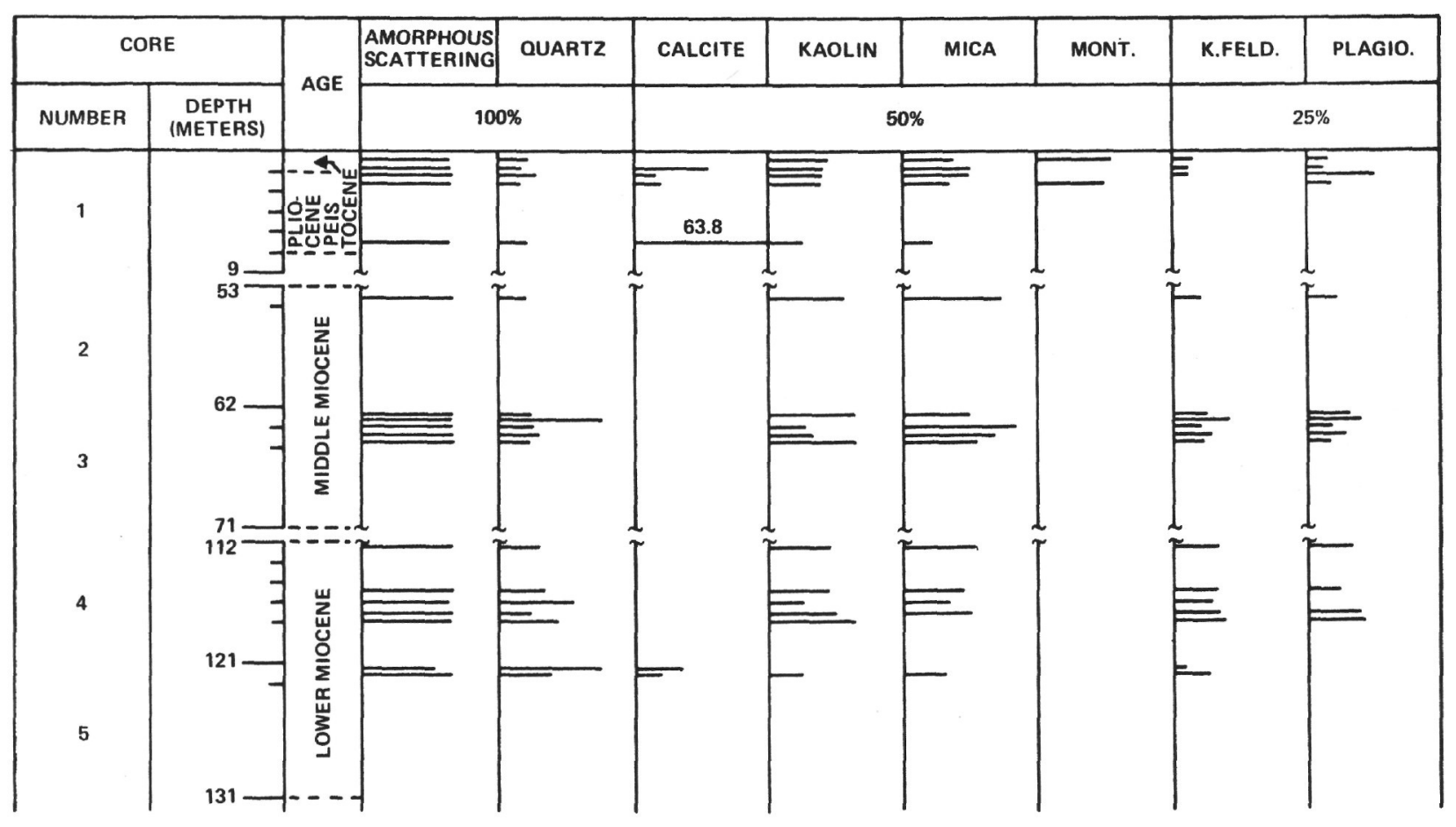

Figure 1. Leg IV, Station 23 (Bulk).

\begin{tabular}{|c|c|c|c|c|c|c|c|c|c|c|c|c|c|c|}
\hline \multicolumn{2}{|c|}{ CORE } & \multirow{2}{*}{ AGE } & $\begin{array}{l}\text { AOOAPPOOUS } \\
\text { SCA }\end{array}$ & OUARTZ & KAOLIN & MONT. & PHILLL. & MICA & DoLom. & SIER. & RHOOO. & K.FELD & PLAGIO & PYRITE \\
\hline NUMBER & $\begin{array}{l}\text { DeETH } \\
\text { (METERSI }\end{array}$ & & $100 x$ & \multicolumn{4}{|c|}{$50 \%$} & $25 \%$ & \multicolumn{6}{|c|}{$10 \%$} \\
\hline 1 & & 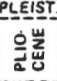 & & & & & & & & & $\overline{\Xi 5}$ & 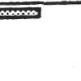 & 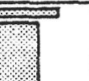 & \\
\hline 3 & & 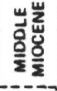 & 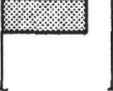 & X & ? & & & ?. & 2. & & & 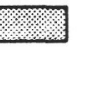 & थ & \\
\hline 4 & & 萢 & $x_{2}$ & & & & & & 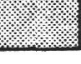 & : & & & & \\
\hline 5 & 131 & قِ & & & & & & & & & & & & \\
\hline
\end{tabular}

Figure 2. Leg IV, Station 23. Composited 2-20 $\mu$ fractions-calc. free. 


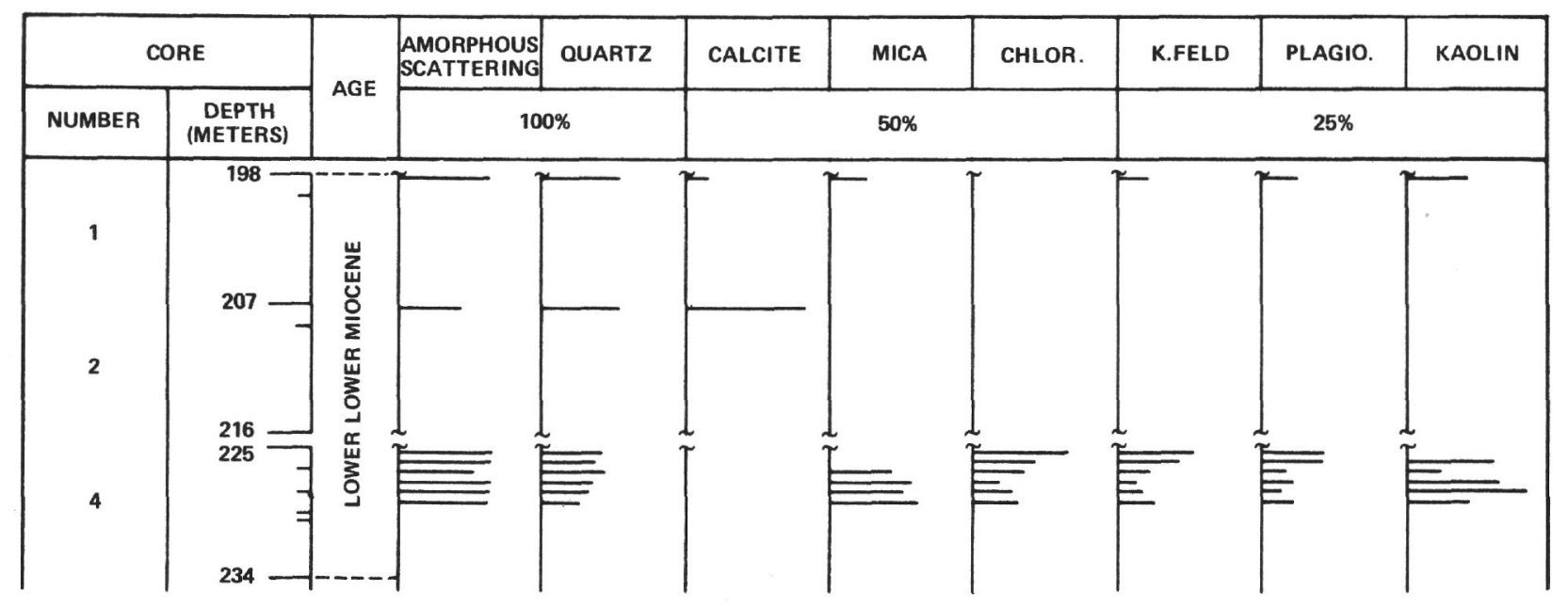

Figure 3. Leg IV, Station 24 (Bulk).

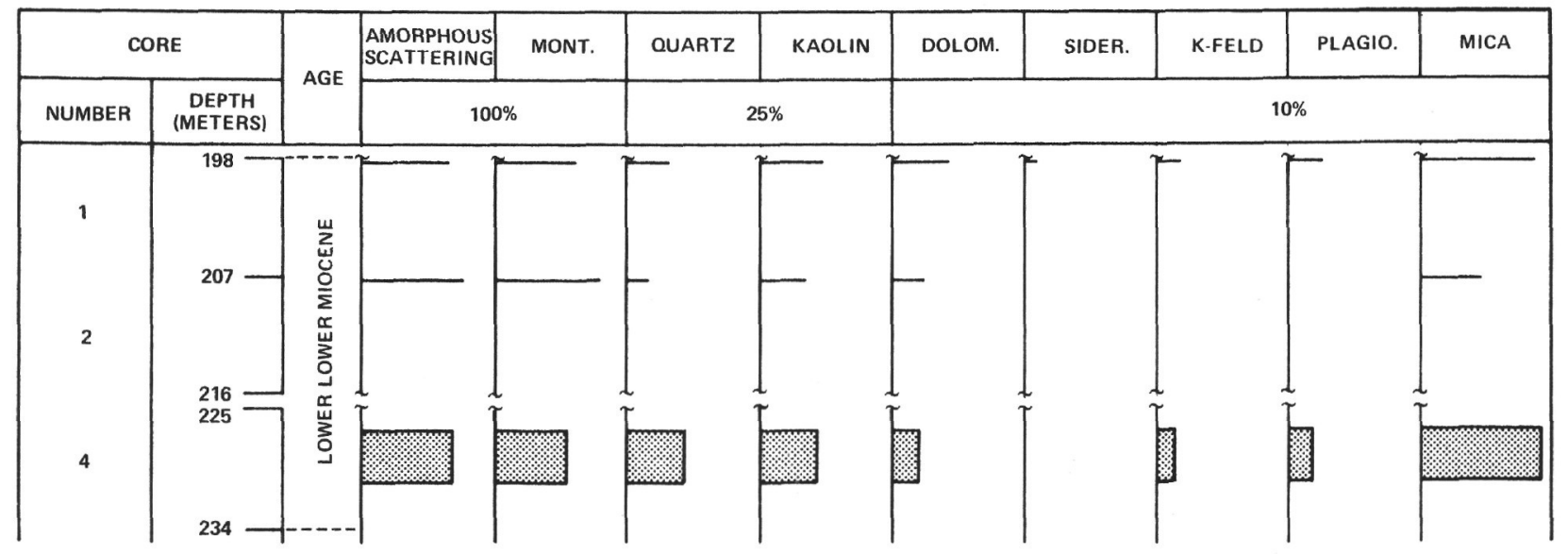

Figure 4. LegIV, Station 24. Composited 2-20 $\mu$ fractions-calc. free. 


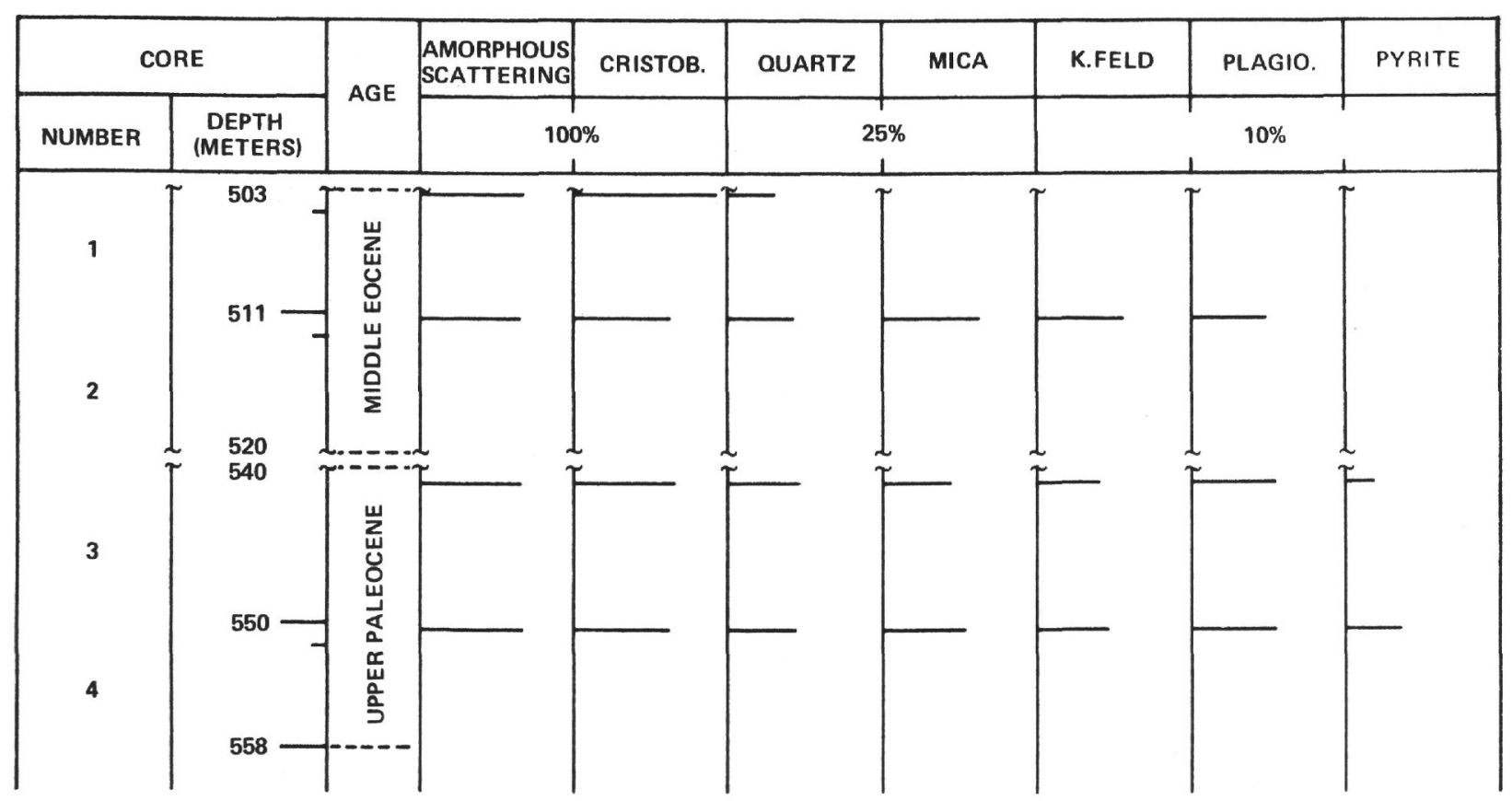

Figure 5. Leg IV, Station 24-A (Bulk).

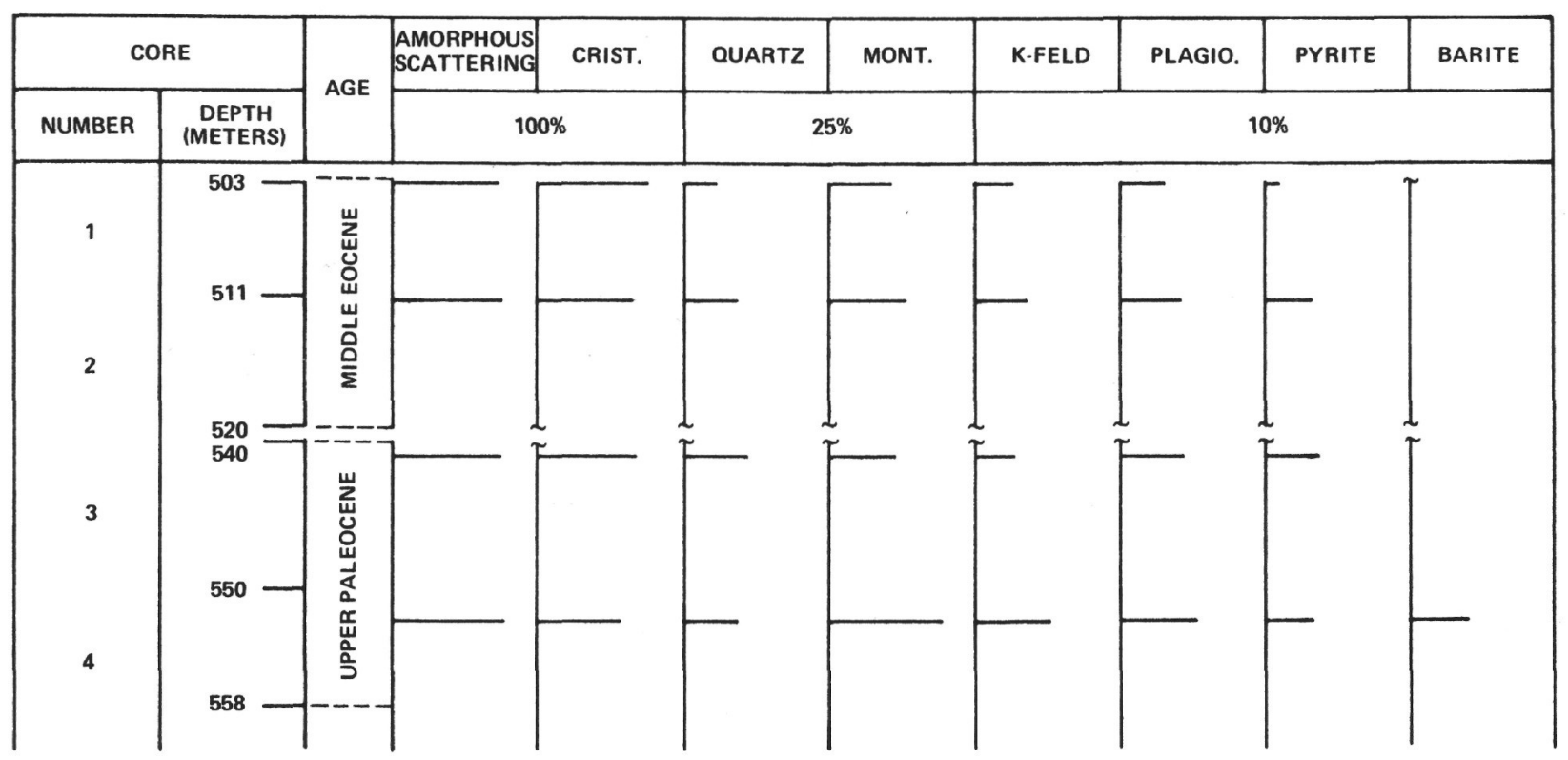

Figure 6. Leg IV, Station 24-A. Composited 2-20 $\mu$ fractions-calc. free. 


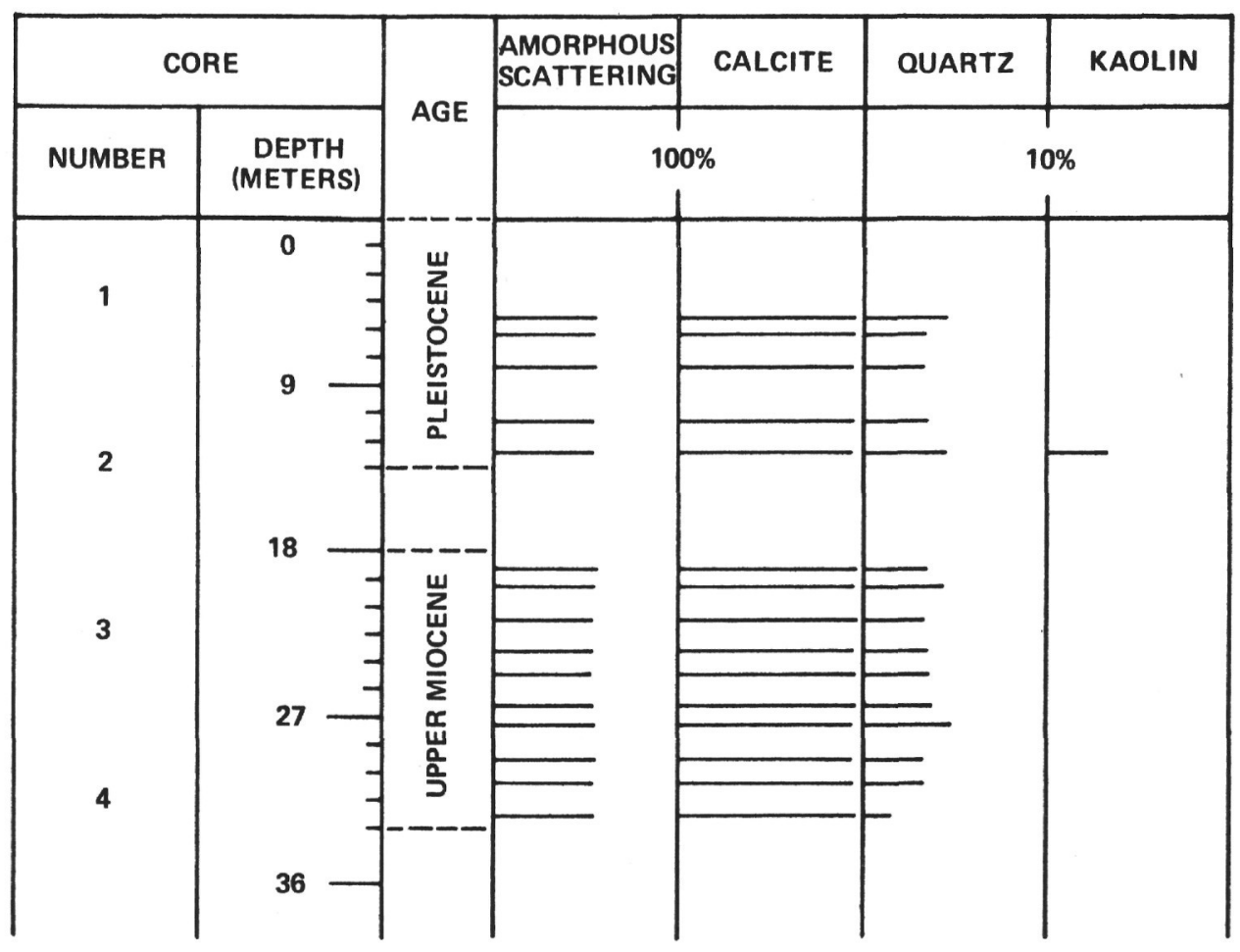

Figure 7. Leg IV, Station 25 (Bulk).

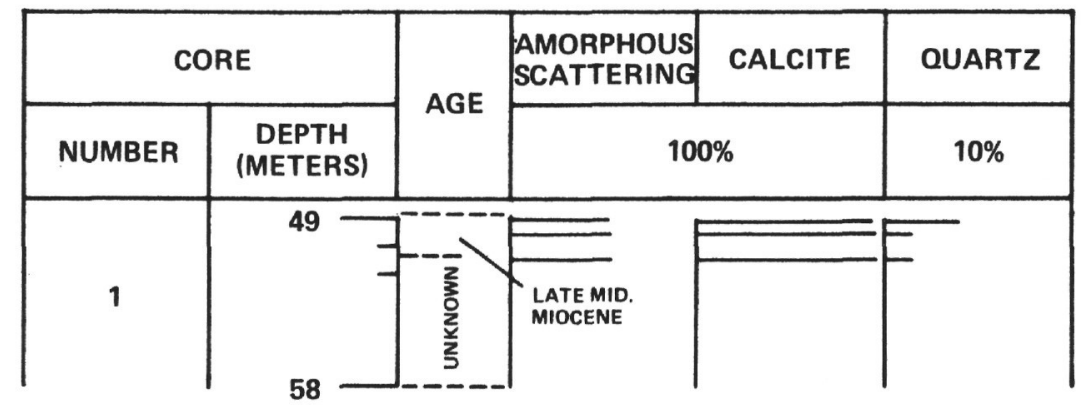

Figure 8. Leg IV, Station 25-A (Bulk). 


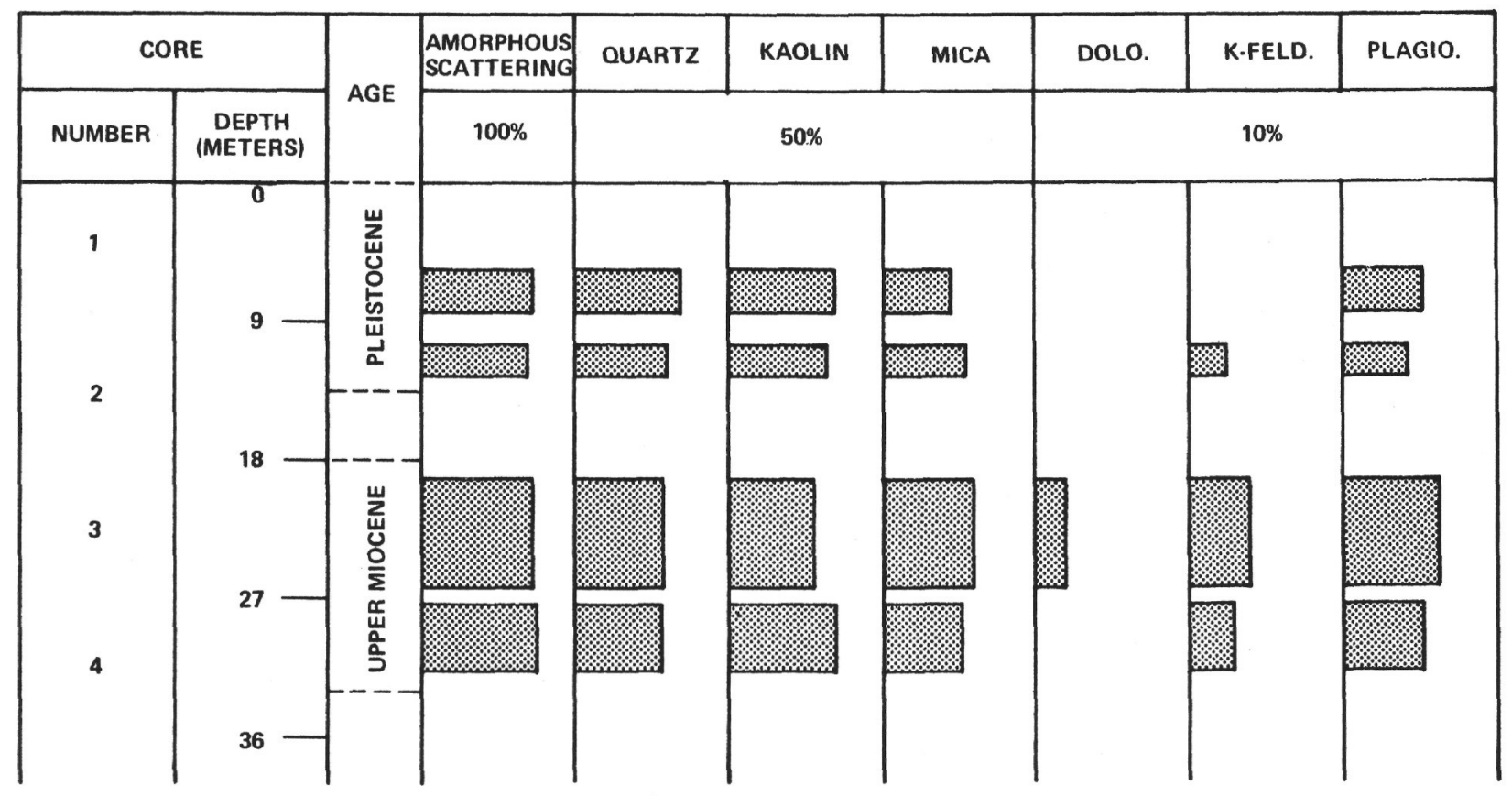

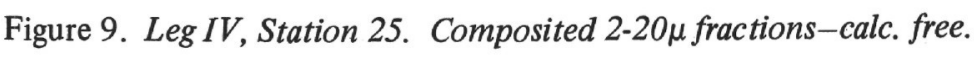

\begin{tabular}{|c|c|c|c|c|c|c|c|c|}
\hline \multicolumn{2}{|c|}{ CORE } & \multirow{2}{*}{ AGE } & $\begin{array}{l}\text { AMORPHOUS } \\
\text { SCATTERING }\end{array}$ & KAOLIN & QUARTZ & MICA & K-FELD & PLAGIO. \\
\hline NUMBER & $\begin{array}{c}\text { DEPTH } \\
\text { (METERS) }\end{array}$ & & $100 \%$ & $50 \%$ & \multicolumn{2}{|c|}{$25 \%$} & \multicolumn{2}{|c|}{$10 \%$} \\
\hline 1 & 58 & 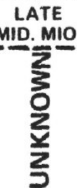 & & & & & & . \\
\hline
\end{tabular}

Figure 10. Leg IV, Station 25-A. Composited 2-20 $\mu$ fractions-calc. free.

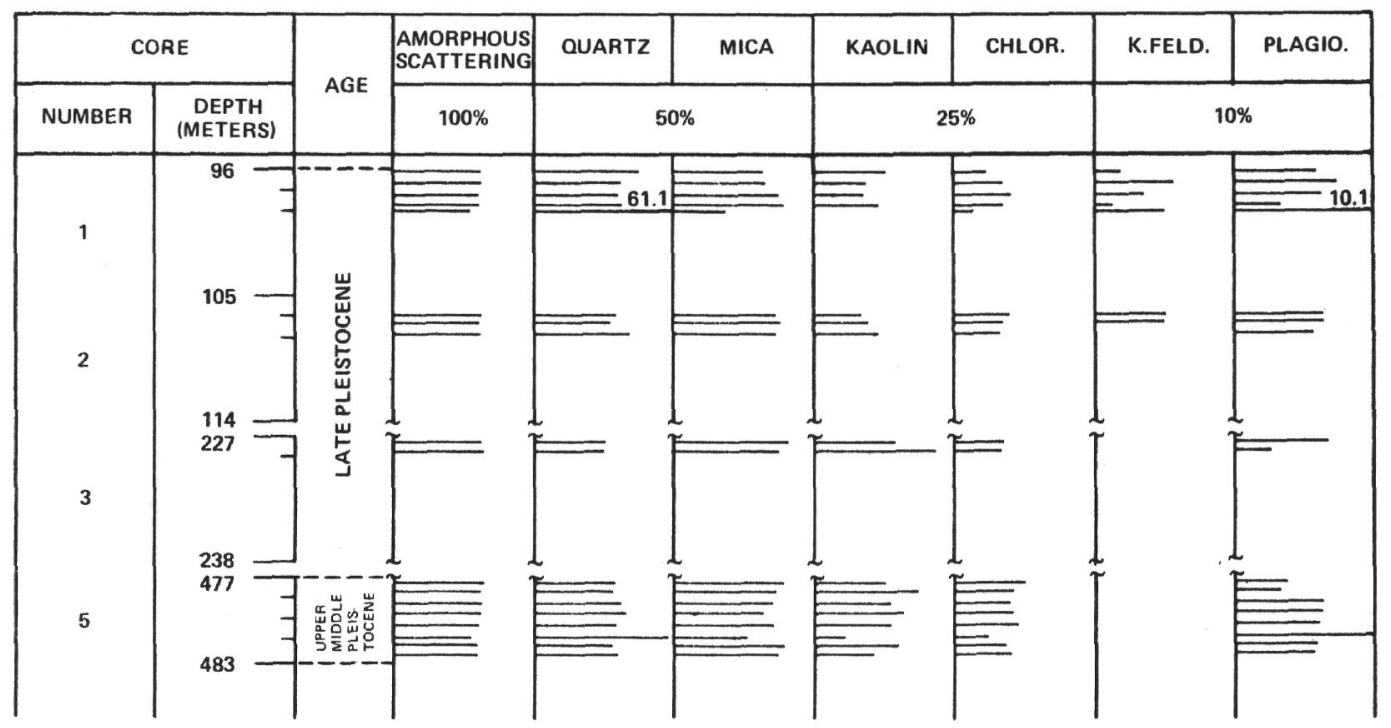

Figure 11. Leg IV, Station 26 (Bulk). 


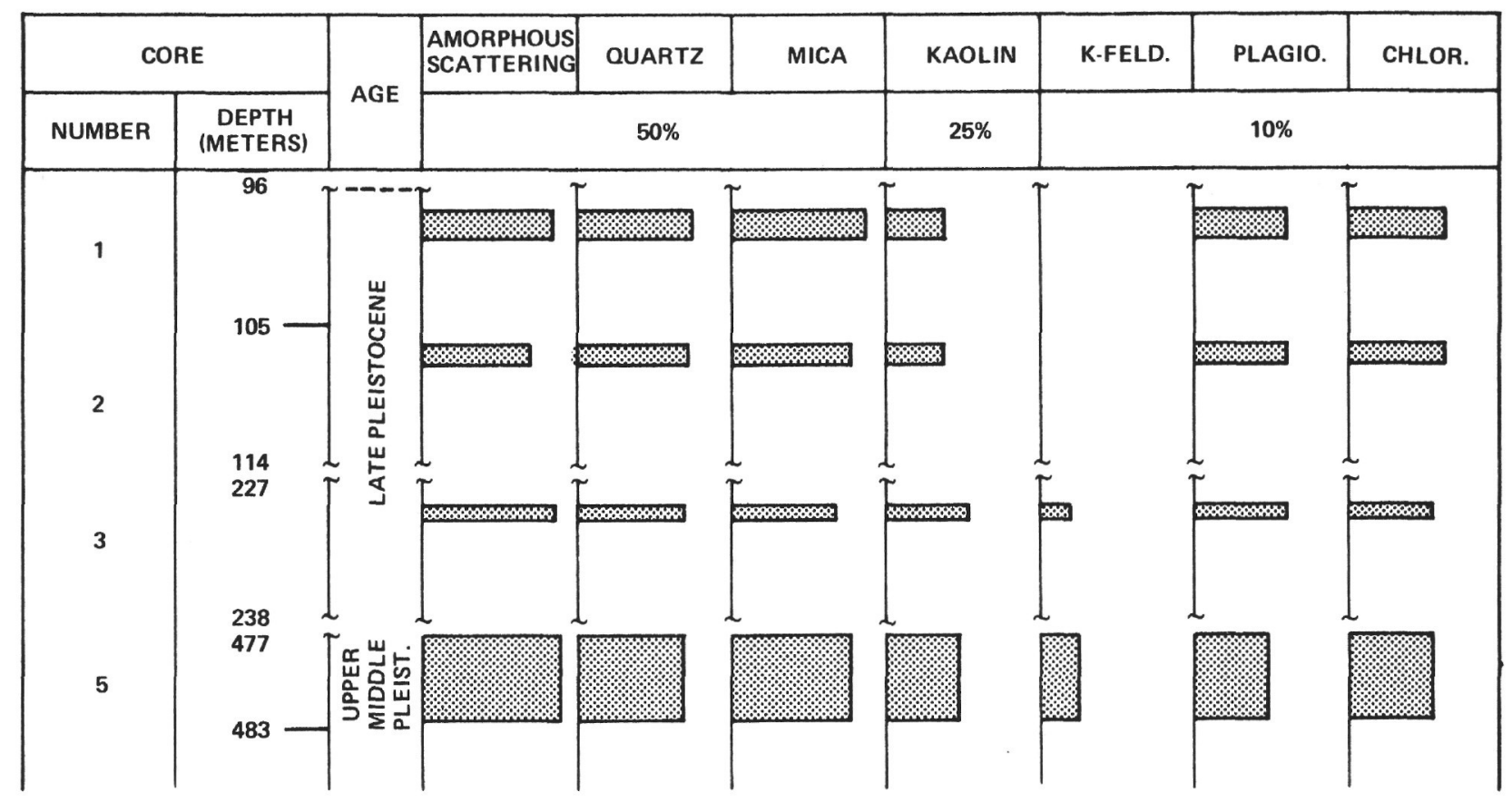

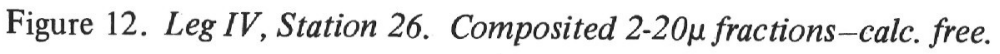

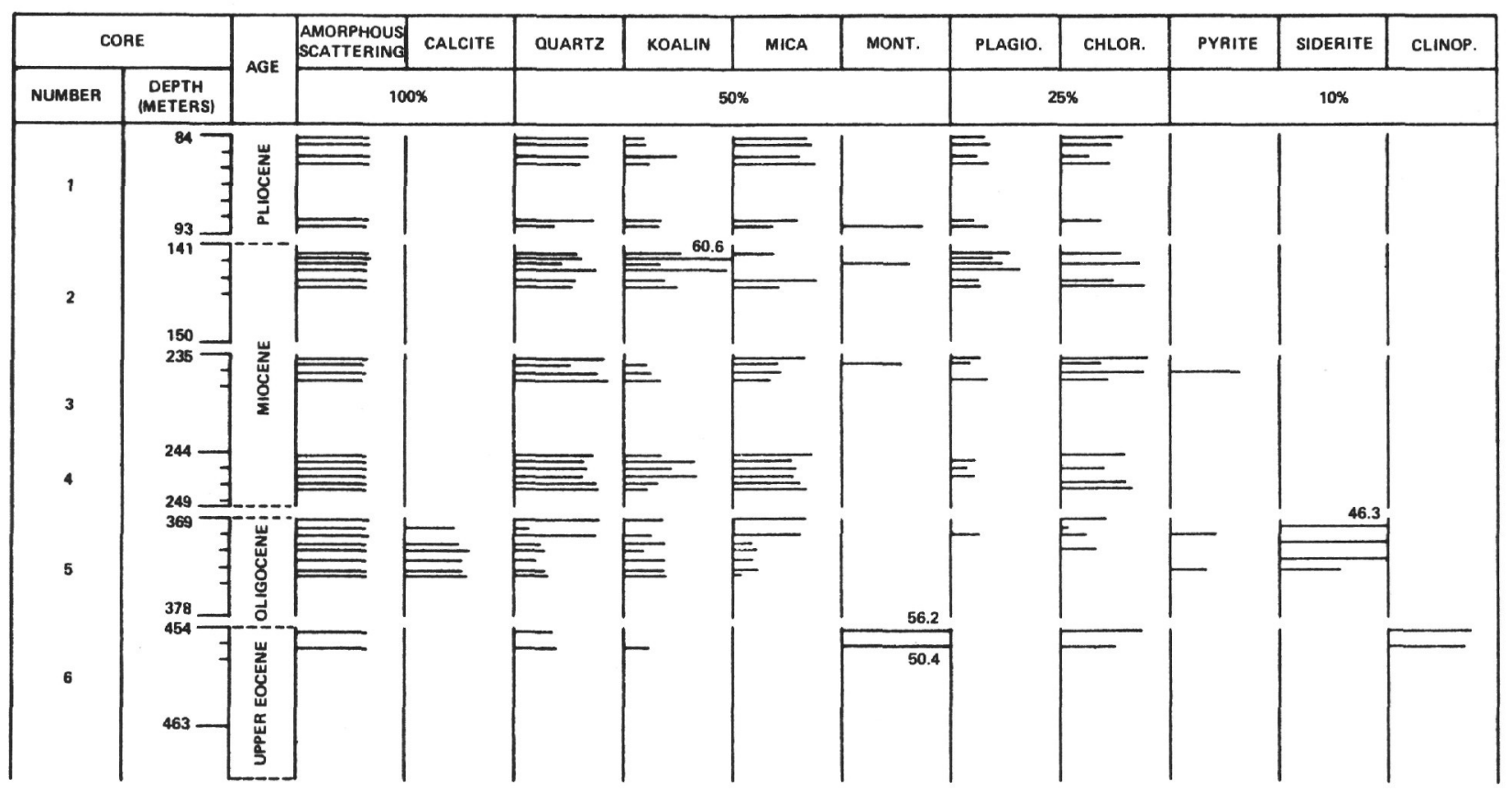

Figure 13. Leg IV, Station 27 (Bulk). 


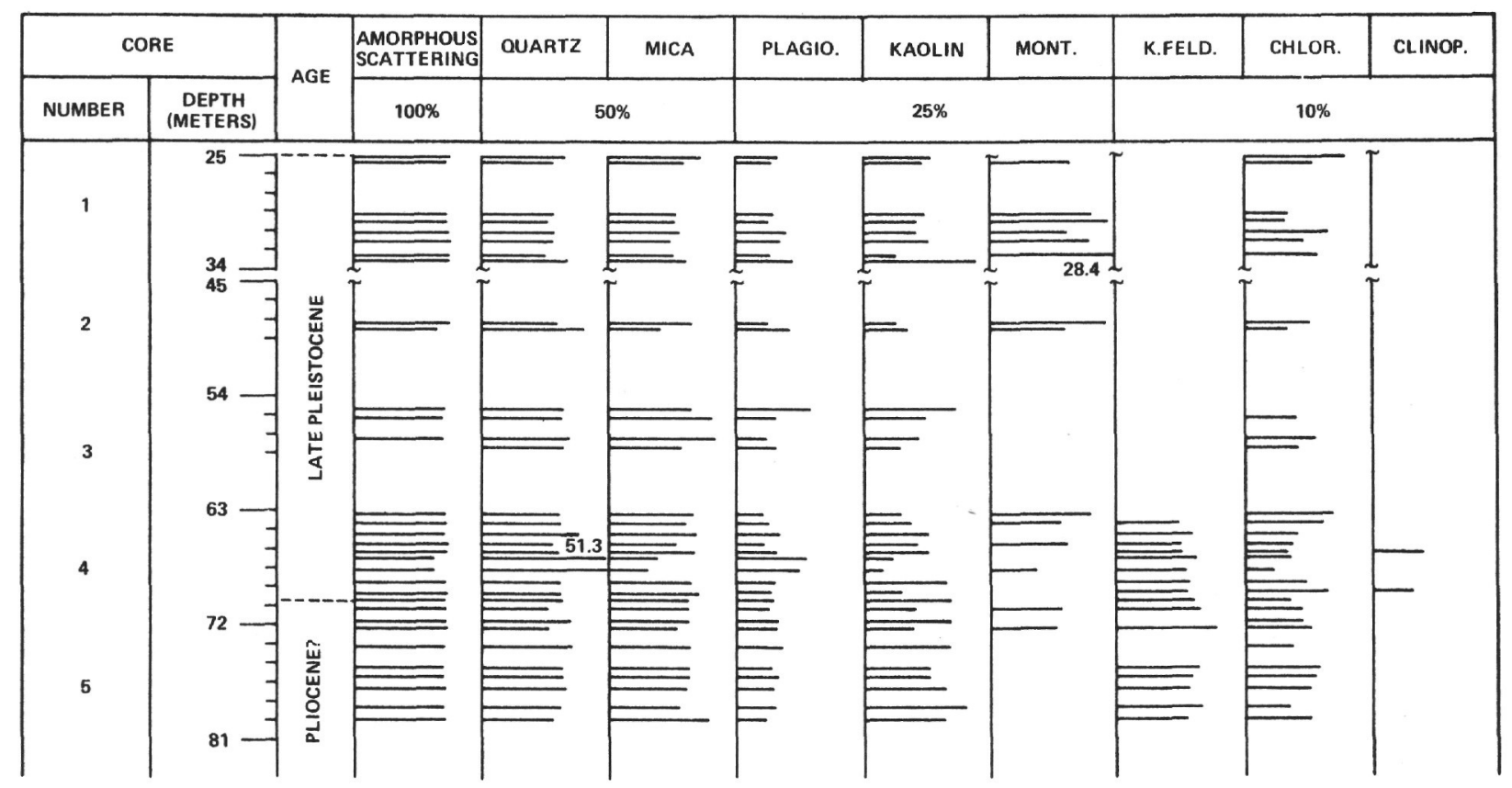

Figure 14. Leg IV, Station 27-A (Bulk).

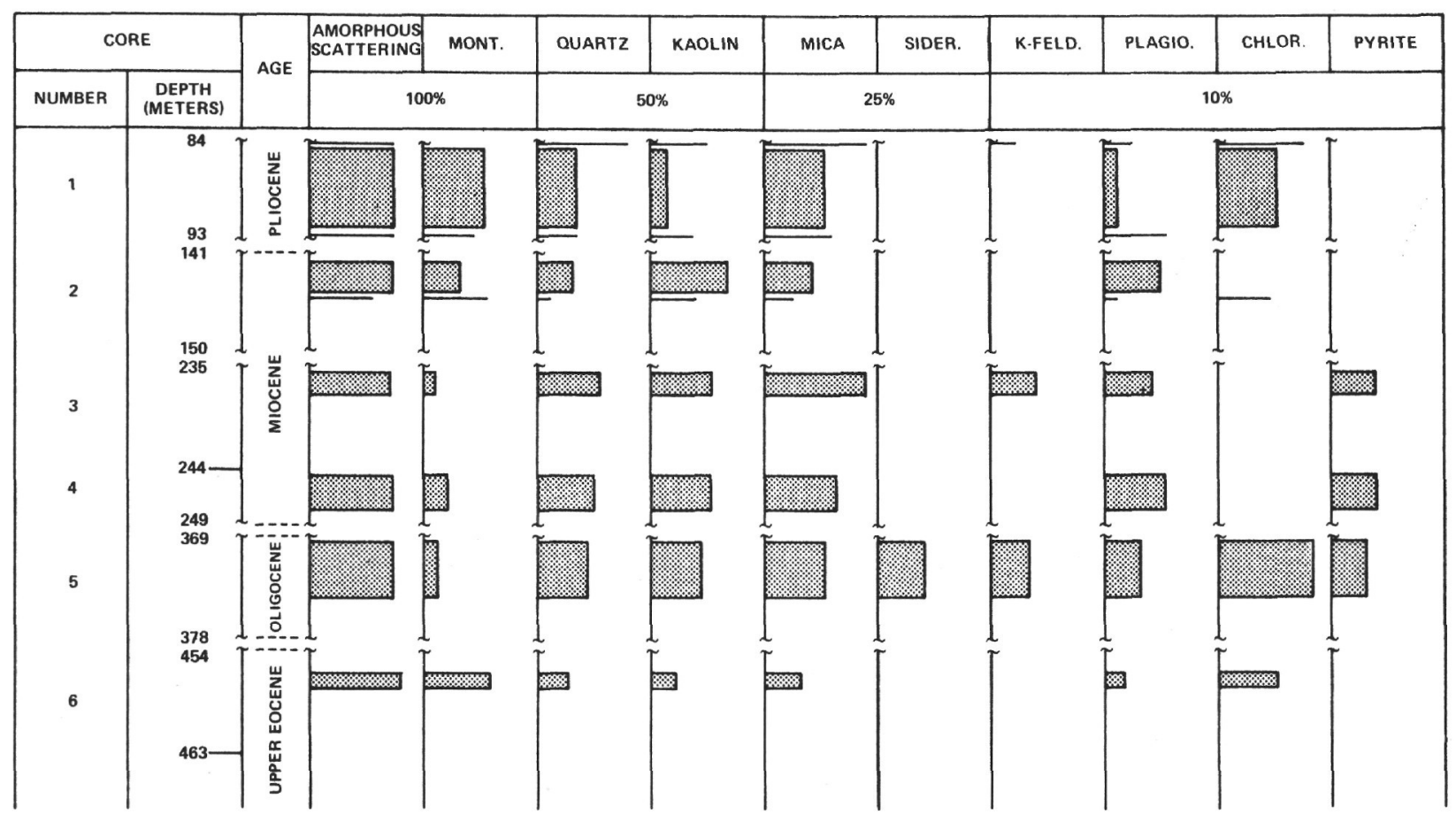

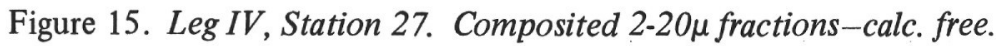




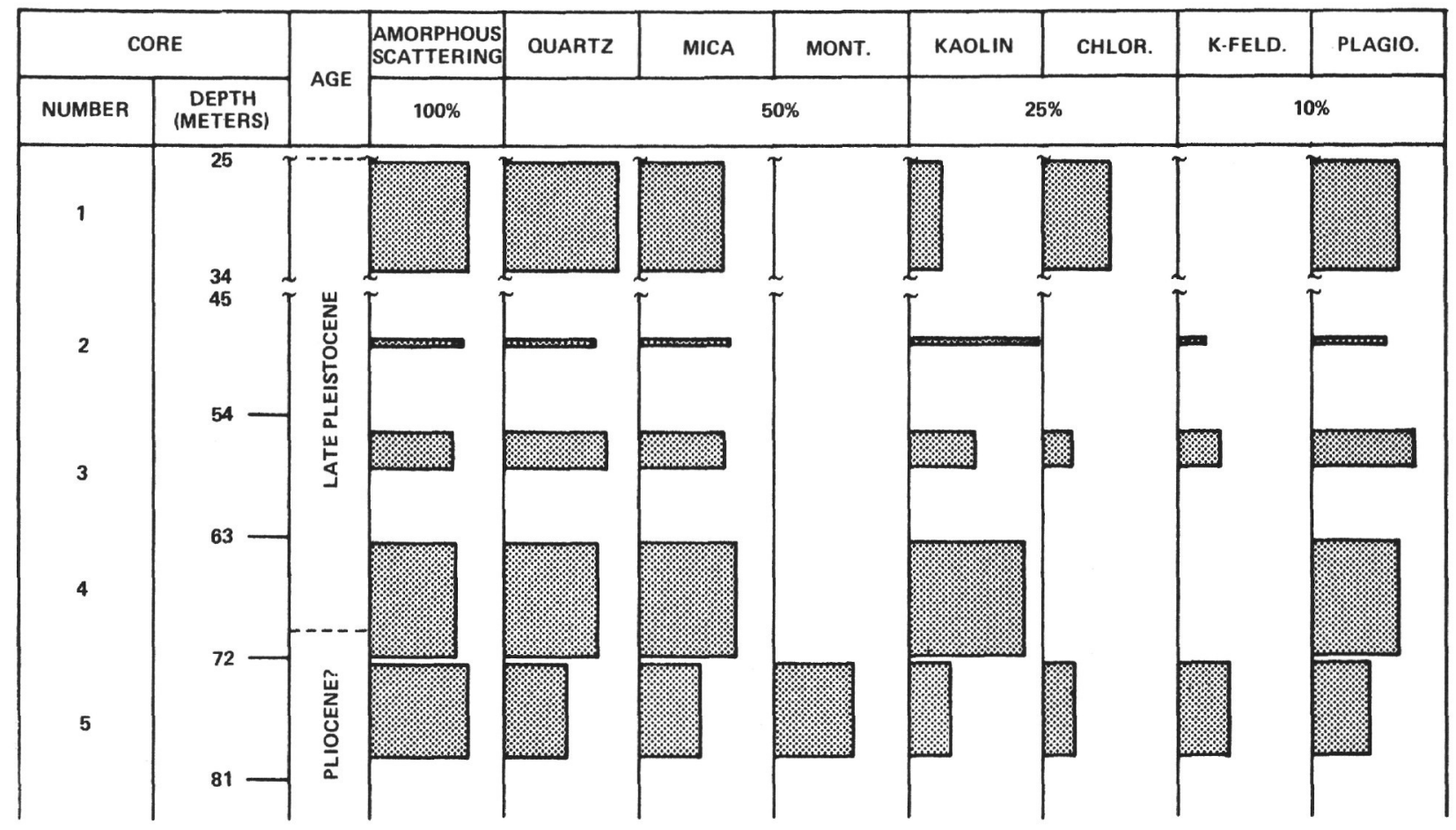

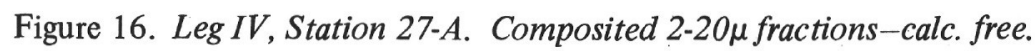

\begin{tabular}{|c|c|c|c|c|c|c|c|c|c|c|c|}
\hline \multicolumn{2}{|c|}{ CORE } & \multirow{2}{*}{ AGE } & \multicolumn{2}{|c|}{\begin{tabular}{l|l} 
AMORPHOUS \\
SCATTERING
\end{tabular}} & QUARTZ & MICA & MONT. & PLAGIO. & KAOLIN & CHLOR. & CLINOP. \\
\hline NUMBER & $\begin{array}{c}\text { DEPTH } \\
\text { (METERS) }\end{array}$ & & & $00 \%$ & & $50 \%$ & & \multicolumn{4}{|c|}{$25 \%$} \\
\hline 2 & 68 & 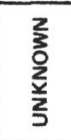 & $\bar{E}$ & $\tau$ & 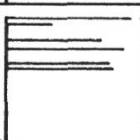 & & & $\sum^{43.0}$ & 25.6 & $=$ & 29.6 \\
\hline 3 & & 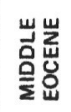 & $\bar{E}$ & $\bar{E}$ & E & & $E$ & & & $\bar{E}$ & $E_{36.4}$ \\
\hline
\end{tabular}

Figure 17. Leg IV, Station 28 (Bulk).

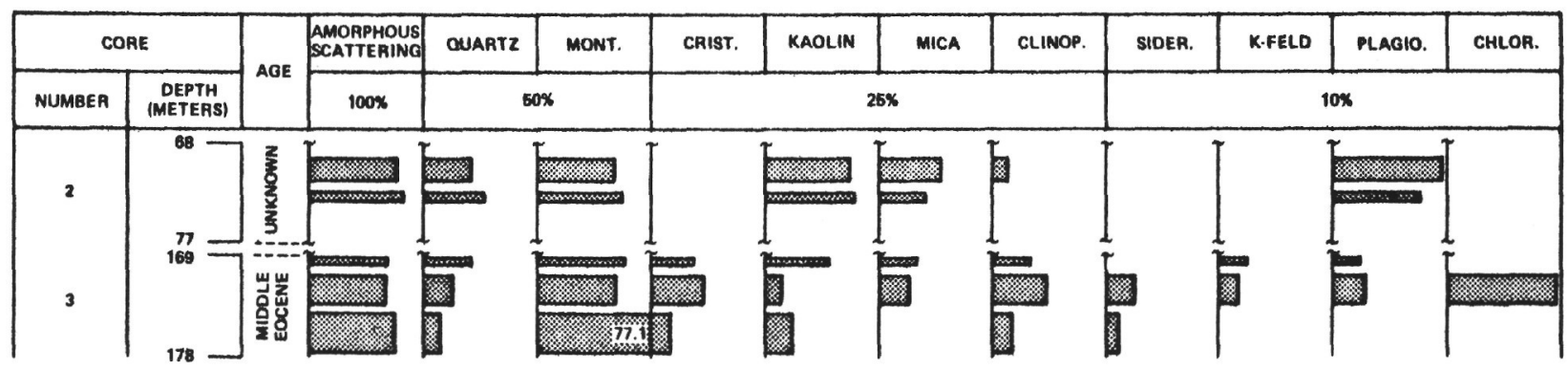

Figure 18. Leg IV, Station 28. Composited 2-20 $\mu$ fractions-calc. free. 


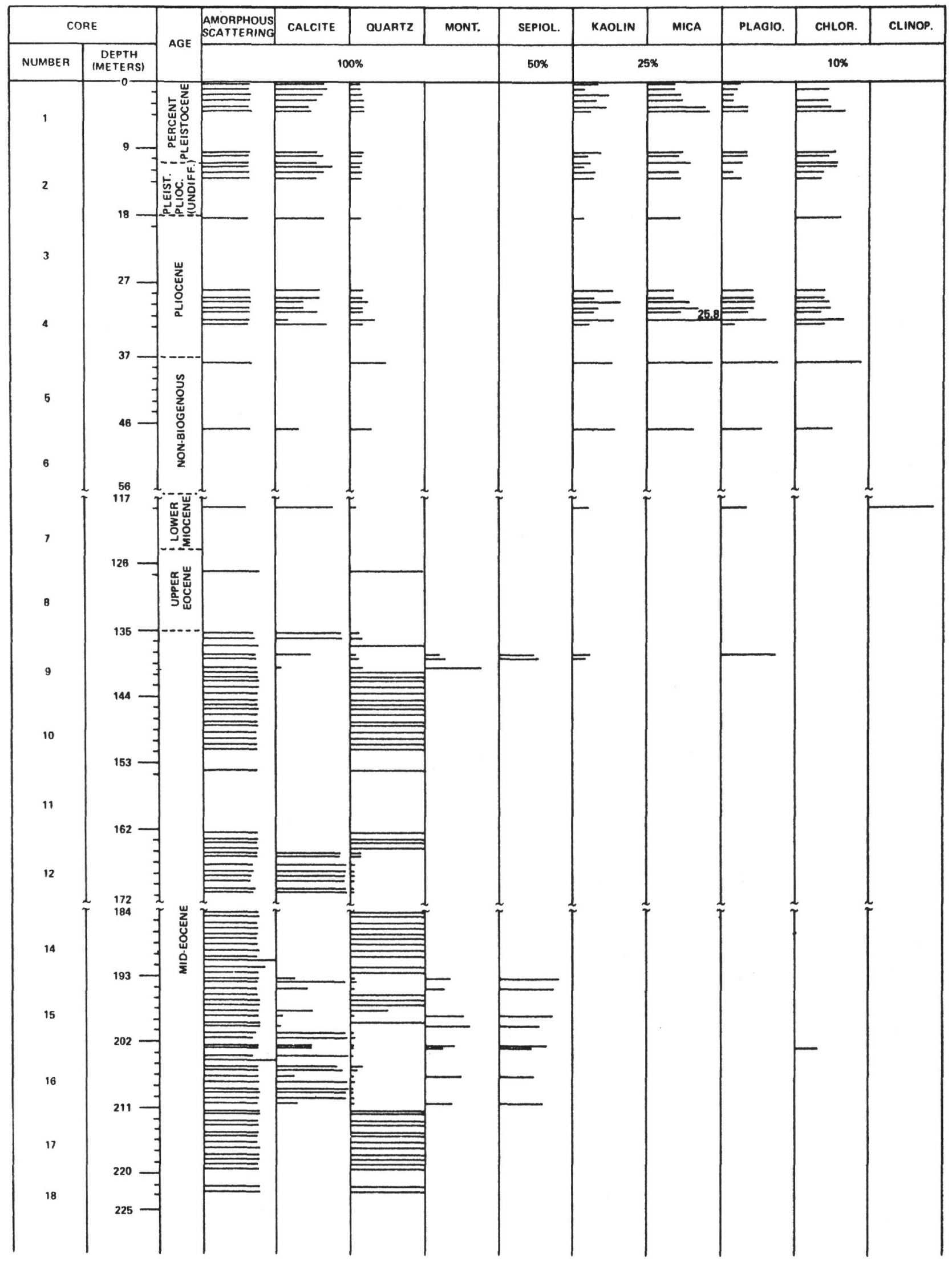

Figure 19. Leg IV, Station 29 (Bulk). 


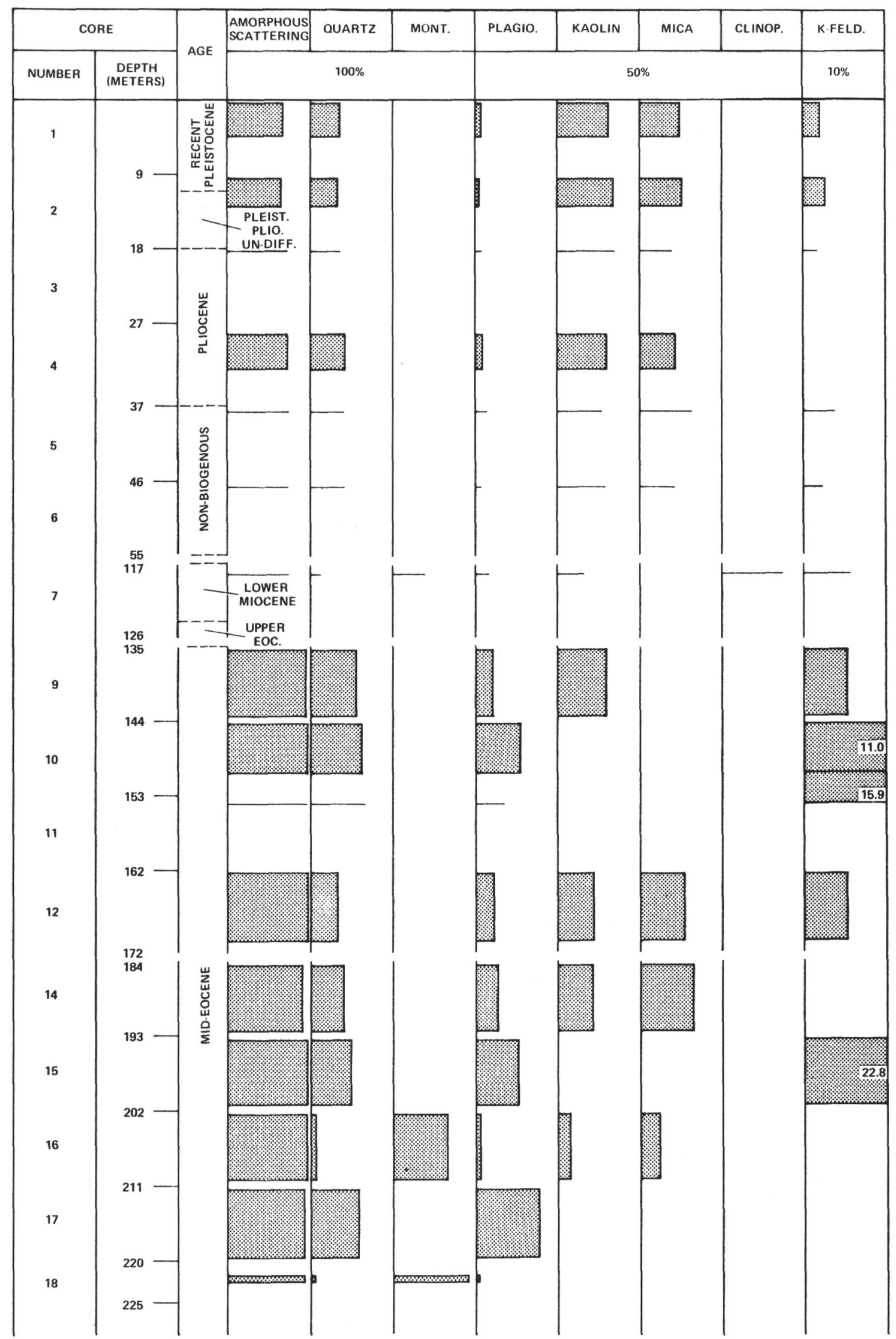

Figure 20. Leg IV, Station 29. Composited 2-20 $\mu$ fractions-calc. free. 


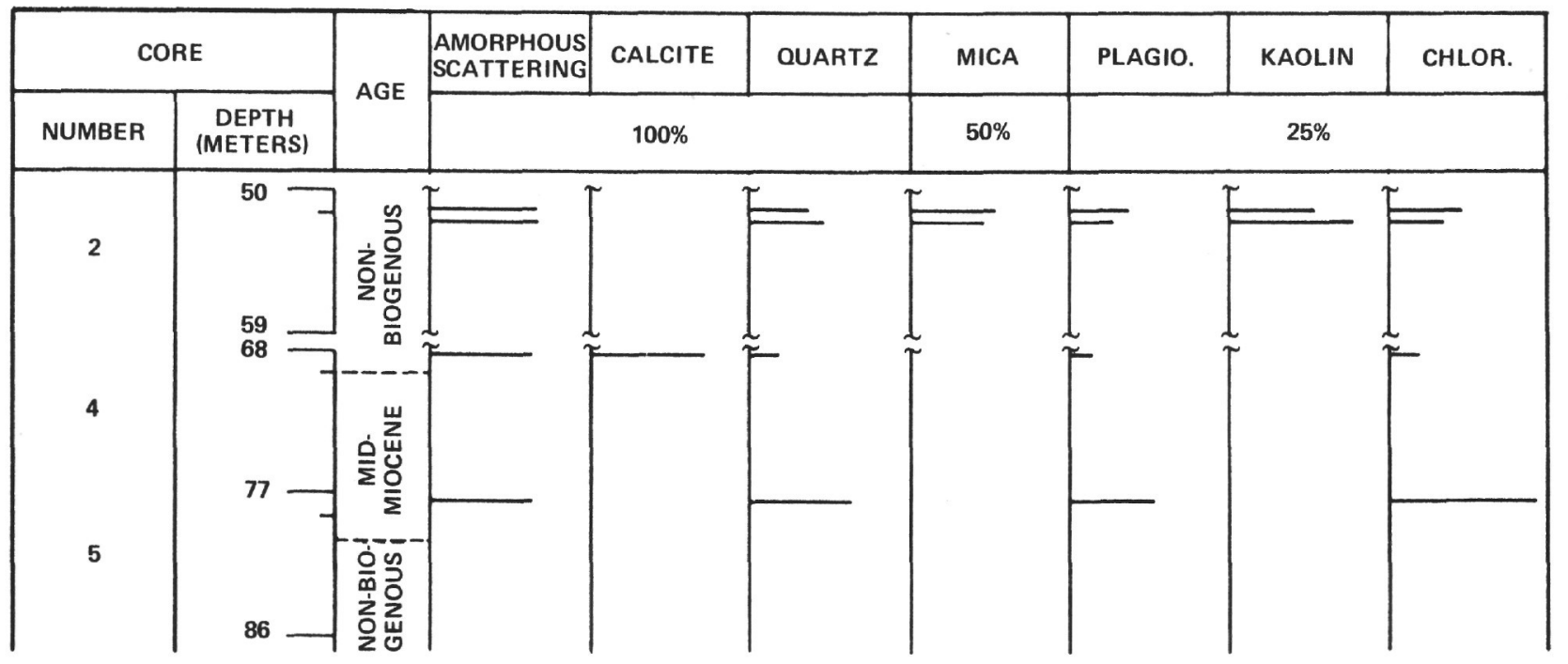

Figure 21. Leg IV, Station 29-A (Bulk).

\begin{tabular}{|c|c|c|c|c|c|c|c|c|c|}
\hline \multicolumn{2}{|c|}{ CORE } & \multirow{2}{*}{ AGE } & $\begin{array}{l}\text { AMORPHOUS } \\
\text { SCATTERING } \\
\end{array}$ & QUARTZ & MONT. & KAOLIN & MICA & PLAGIO. & CHLOR. \\
\hline NUMBER & $\begin{array}{c}\text { DEPTH } \\
\text { (METERS) }\end{array}$ & & $100 \%$ & \multicolumn{2}{|c|}{$50 \%$} & \multicolumn{2}{|c|}{$25 \%$} & \multicolumn{2}{|c|}{$10 \%$} \\
\hline 2 & 59 & 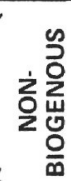 & Thensm & D. & & 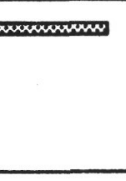 & 资 & 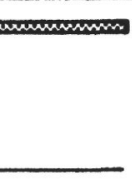 & 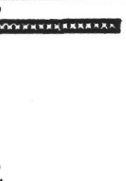 \\
\hline 4 & $77-$ & $\frac{1}{\Sigma} \frac{2}{\underline{u}}$ & & & & & & & \\
\hline 5 & 86 & 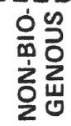 & & & & & & & \\
\hline
\end{tabular}

Figure 22. Leg IV, Station 29-A. Composited 2-20 $\mu$ fractions-calc. free. 


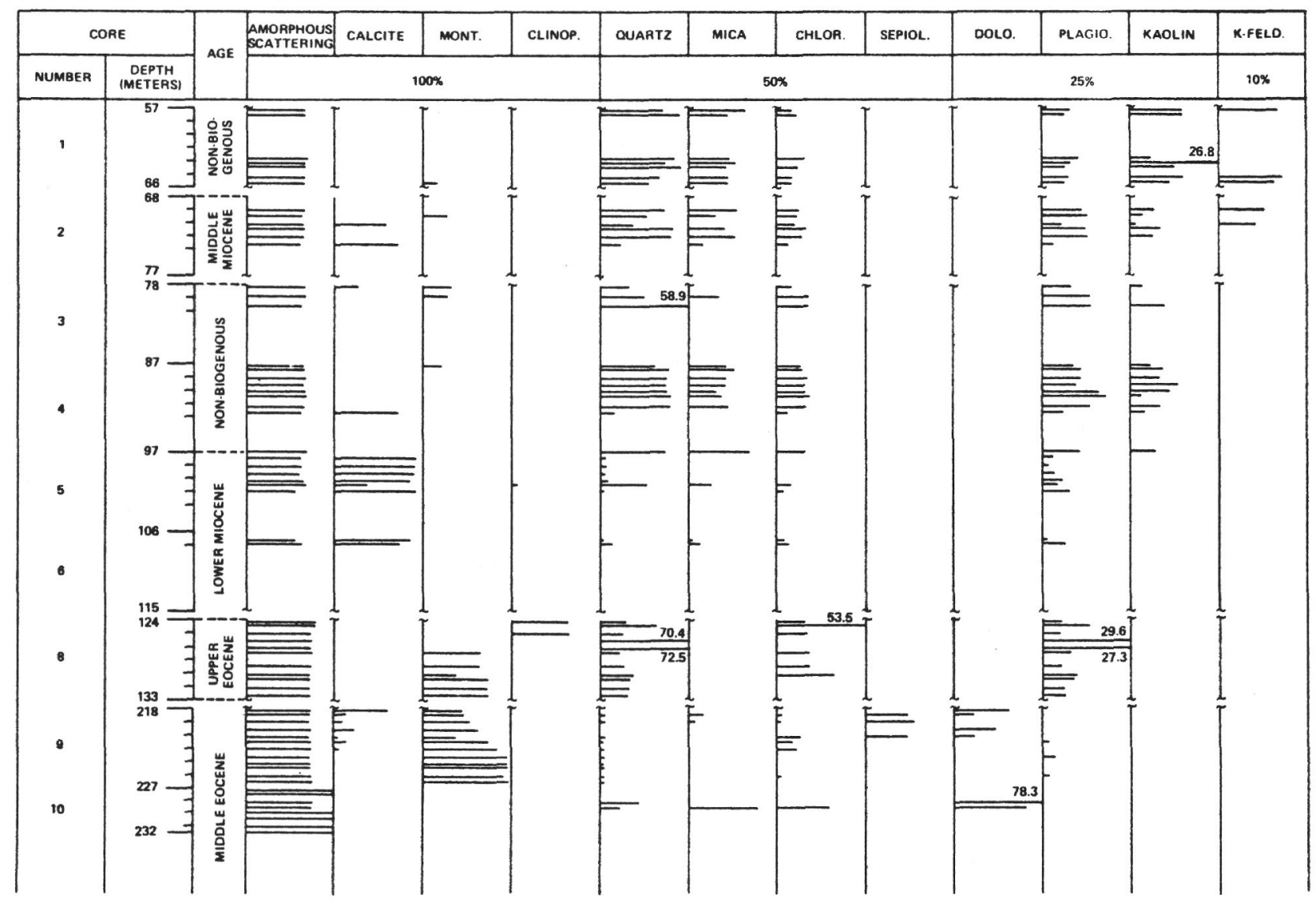

Figure 23. Leg IV, Station 29-B (Bulk). 


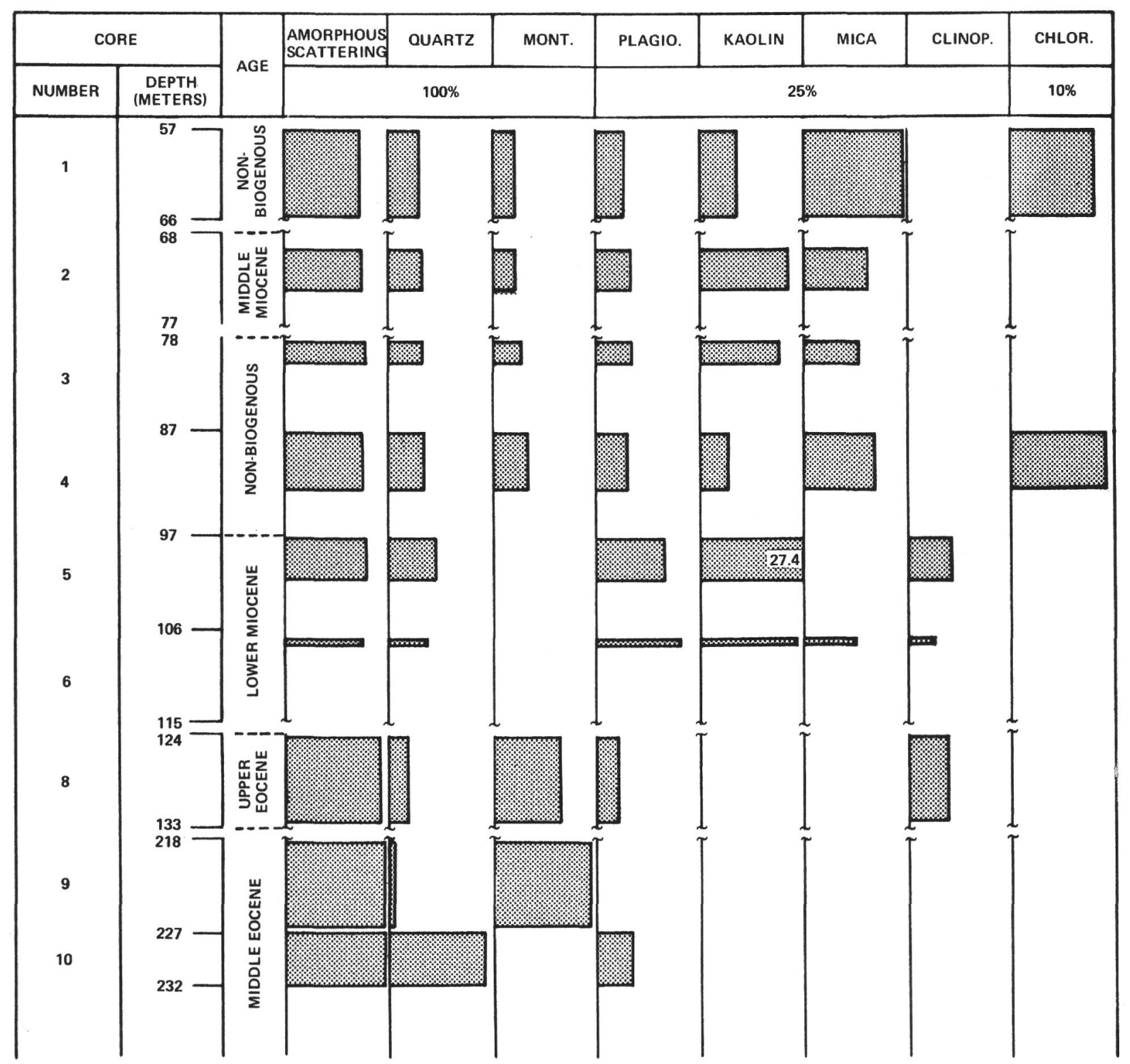

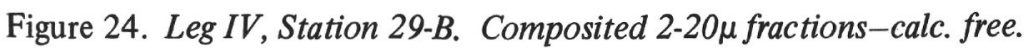




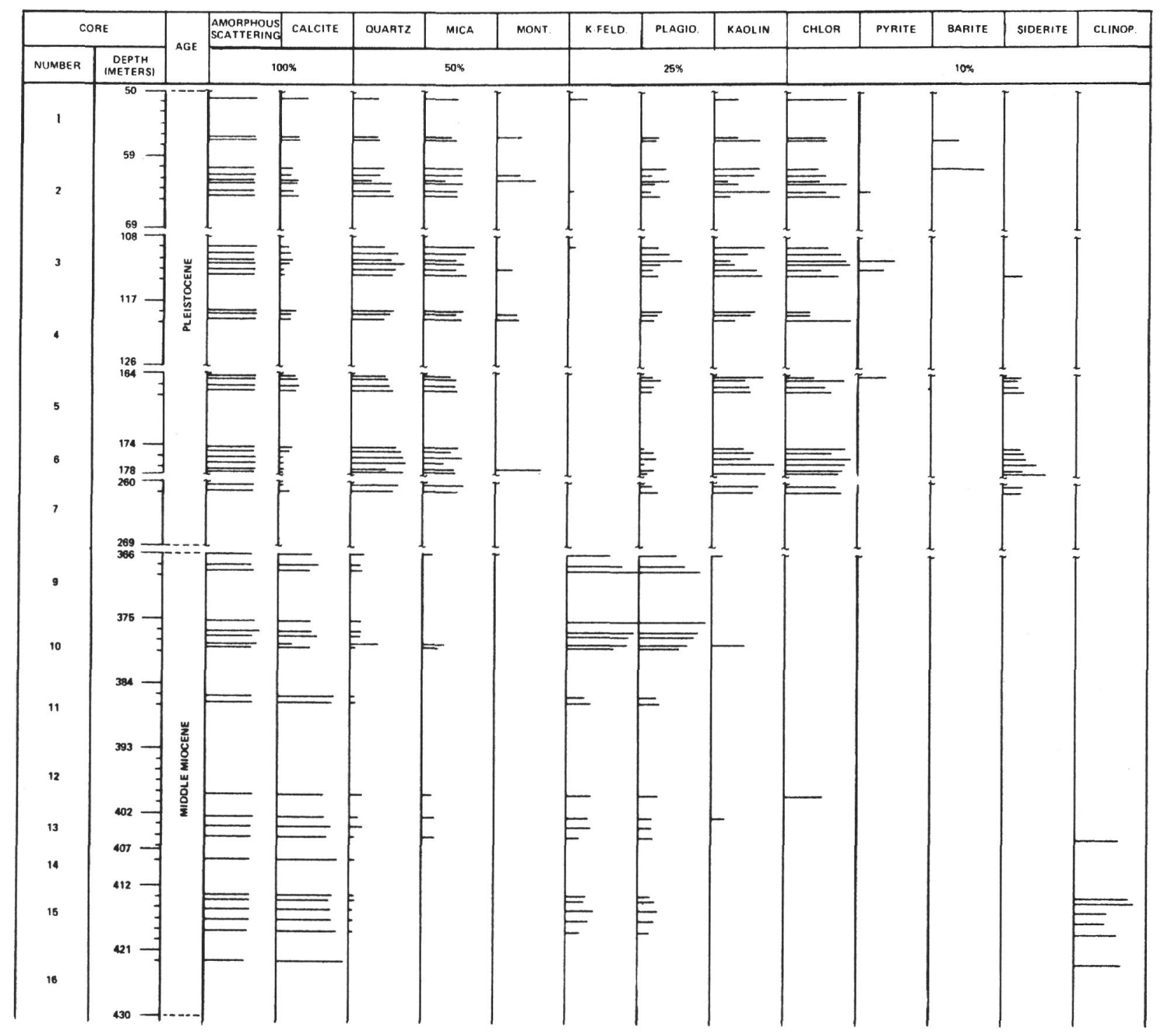

Figure 25. Leg IV, Station 30 (Bulk). 


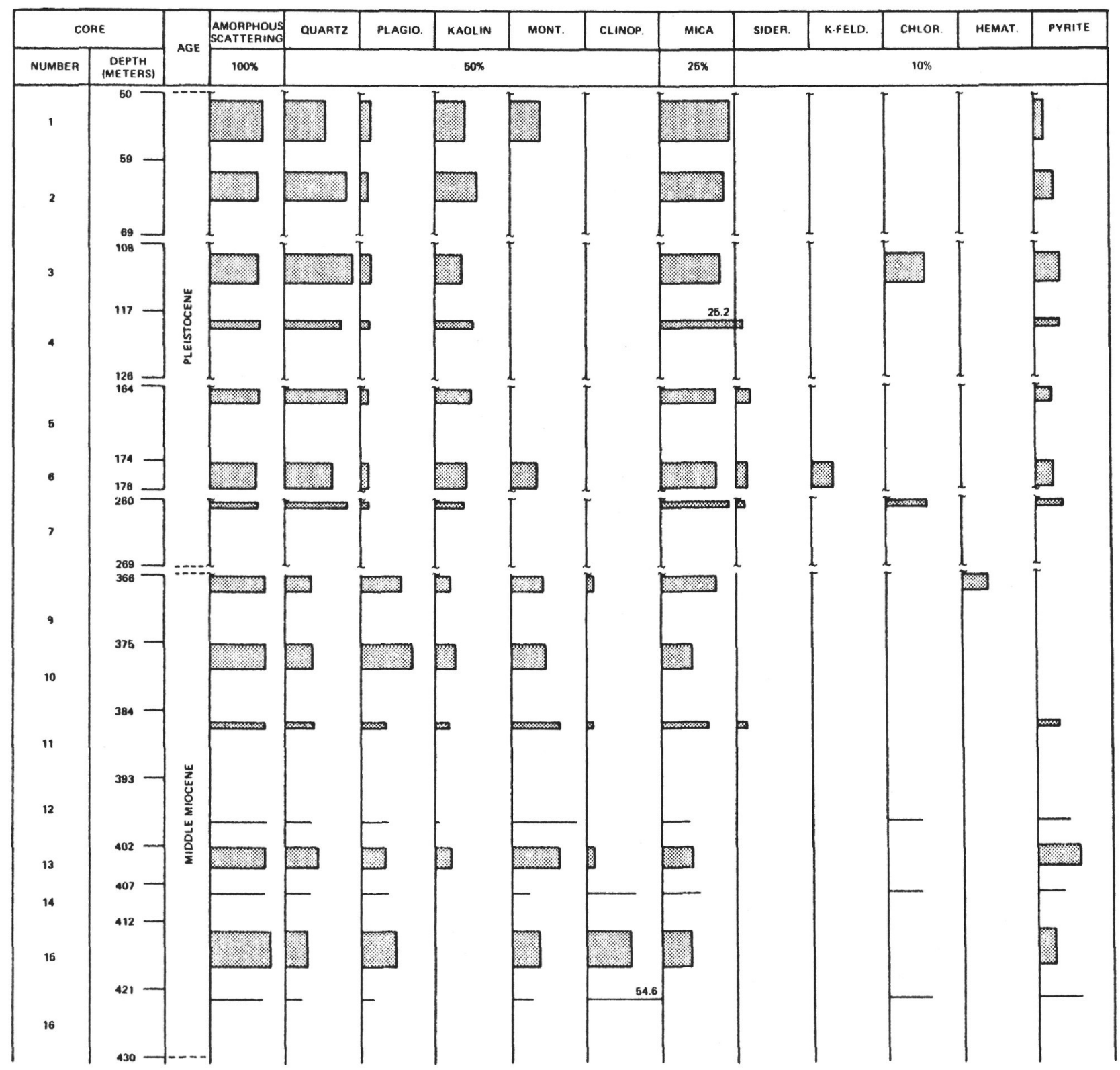

Figure 26. Leg IV, Station 30. Composited 2-20 $\mu$ fractions-calc. free. 


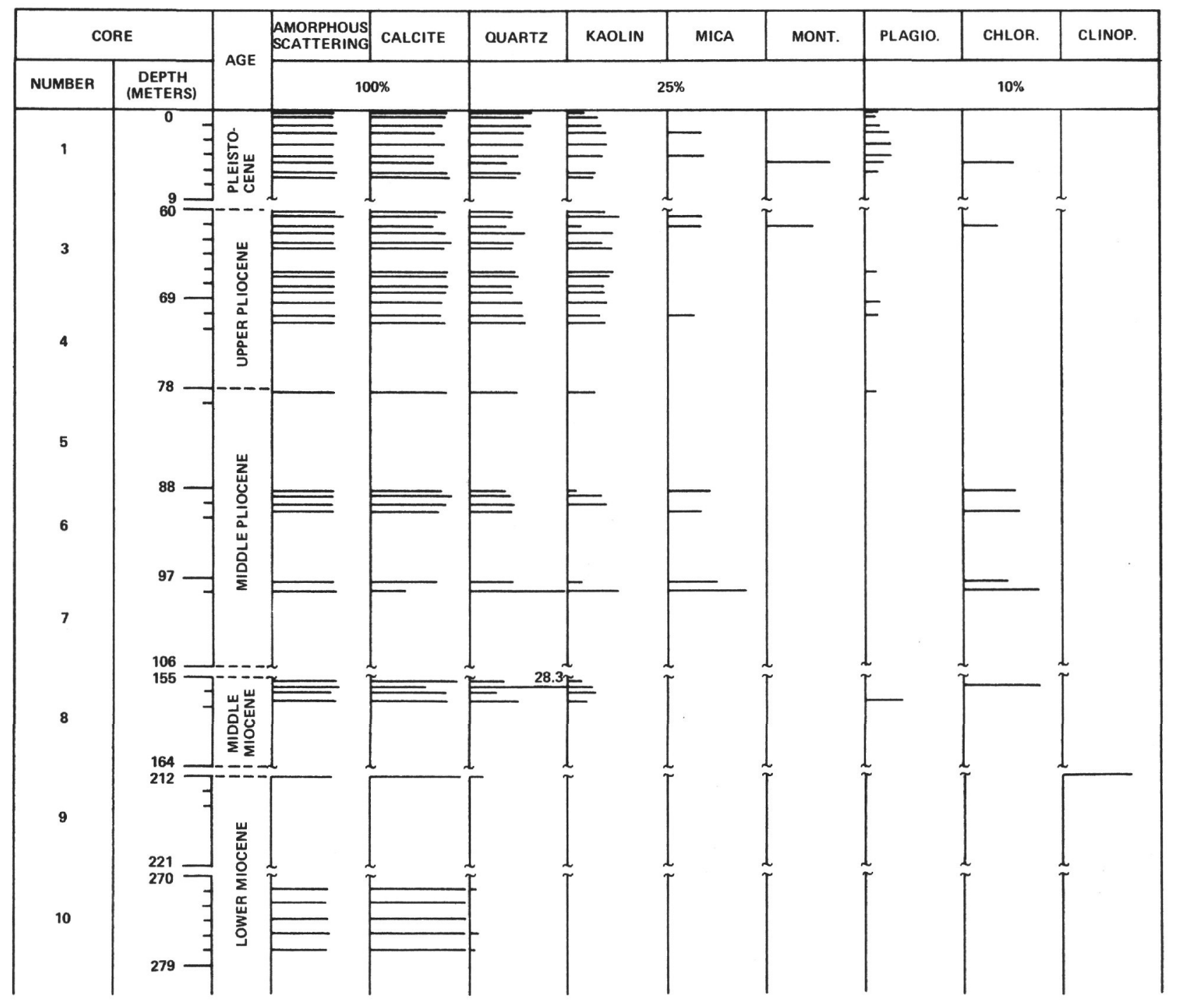

Figure 27. Leg IV, Station 31 (Bulk). 


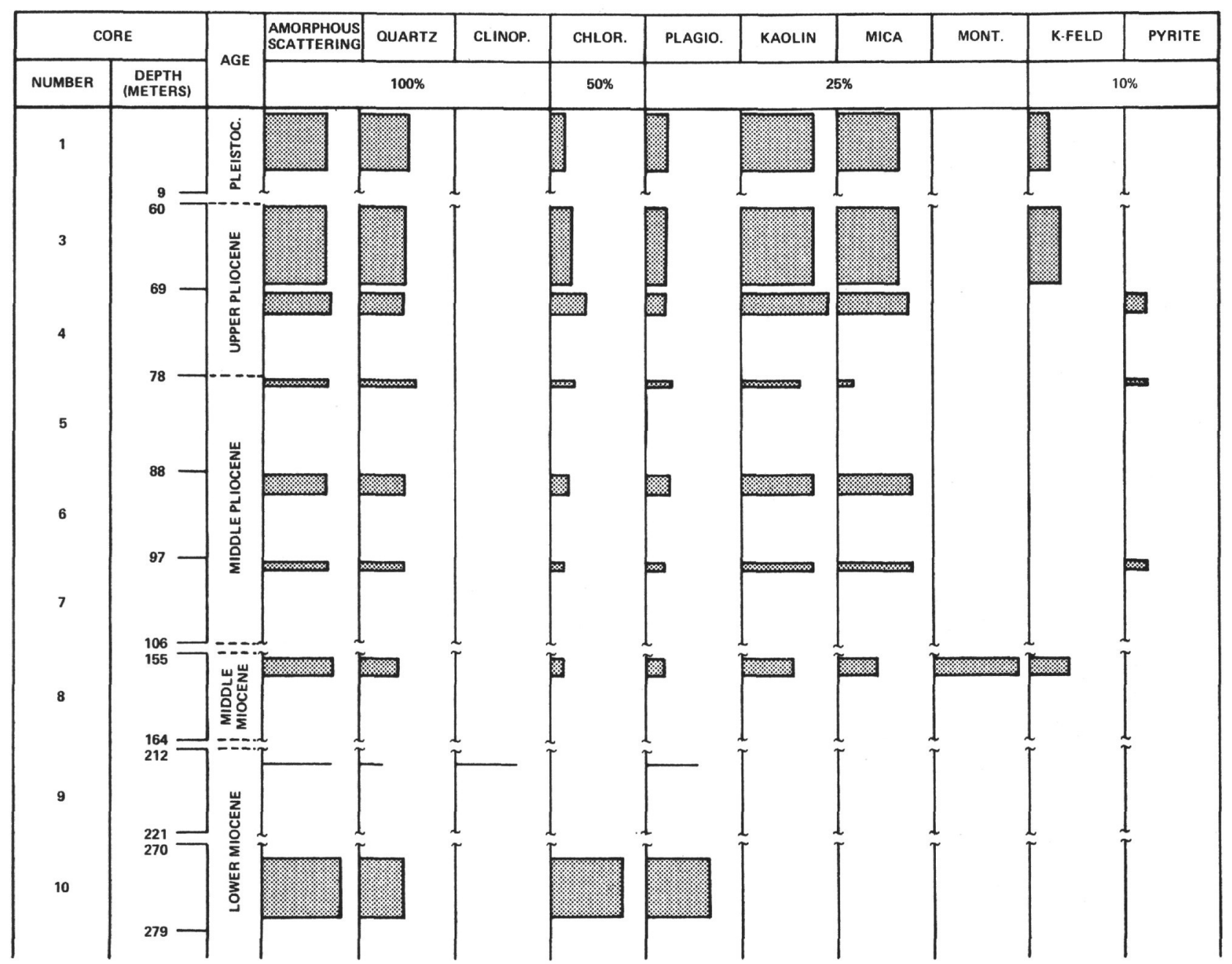

Figure 28. Leg IV, Station 31. Composited 2-20u fractions-calc. free. 
TABLE 1

X-ray Diffraction Analysis of Samples from Leg 4: Bulk Samples ${ }^{\mathrm{a}}$

\begin{tabular}{|c|c|c|c|c|c|c|c|c|c|c|c|c|}
\hline \multicolumn{13}{|l|}{ Hole 23} \\
\hline Core & $\begin{array}{l}\text { Depth Below } \\
\text { Sea Floor } \\
\text { (m) }\end{array}$ & Section & $\begin{array}{l}\text { Depth In } \\
\text { Section } \\
(\mathrm{cm})\end{array}$ & Diff. & $\begin{array}{c}\% \\
\text { Amorph. } \\
\text { Scattering }\end{array}$ & Calc. & Quar. & $\mathrm{K}-\mathrm{Fe}$ & Plag. & Kaol. & Mica & Mont. \\
\hline 1 & $0-9$ & 1 & 69 & 87.8 & 64.1 & 0.0 & 22.2 & 4.0 & 4.2 & 22.2 & 19.7 & 27.7 \\
\hline 1 & & & $112-113$ & 88.7 & 66.8 & 28.9 & 17.3 & 3.6 & 3.8 & 20.8 & 25.6 & 0.0 \\
\hline 1 & & 2 & 8 & 88.9 & 67.4 & 8.1 & 29.1 & 3.7 & 13.0 & 21.0 & 25.0 & 0.0 \\
\hline 1 & & & 75 & 89.0 & 67.6 & 11.3 & 18.6 & 0.0 & 5.6 & 20.1 & 17.9 & 26.4 \\
\hline 1 & & 5 & NDG & 87.8 & 64.1 & 63.8 & 12.3 & 0.0 & 0.0 & 13.3 & 10.6 & 0.0 \\
\hline 2 & $53-62$ & 1 & 80 & 89.1 & 67.9 & 0.0 & 22.4 & 5.8 & 6.3 & 28.7 & 36.8 & 0.0 \\
\hline 3 & $62-71$ & 2 & 22 & 89.2 & 68.2 & 0.0 & 26.2 & 6.8 & 8.4 & 33.5 & 25.1 & 0.0 \\
\hline 3 & & & $66-71$ & 89.0 & 67.6 & 0.0 & 78.3 & 11.3 & 10.4 & 0.0 & 0.0 & 0.0 \\
\hline 3 & & & 130 & 89.4 & 68.8 & 0.0 & 29.3 & 5.8 & 5.1 & 15.6 & 44.2 & 0.0 \\
\hline 3 & & 3 & $16-18$ & 89.2 & 68.2 & 0.0 & 31.7 & 7.7 & 7.9 & 17.4 & 35.2 & 0.0 \\
\hline 3 & & & 82 & 89.6 & 69.4 & 0.0 & 26.1 & 6.2 & 4.6 & 34.3 & 28.6 & 0.0 \\
\hline 4 & $112-121$ & 1 & 4-6 & 89.3 & 68.5 & 0.0 & 32.1 & 8.4 & 8.7 & 24.4 & 26.4 & 0.0 \\
\hline 4 & & 3 & 45 & 89.2 & 68.2 & 0.0 & 37.2 & 8.5 & 6.7 & 23.8 & 23.8 & 0.0 \\
\hline 4 & & & $134-136$ & 88.2 & 65.3 & 0.0 & 59.2 & 7.7 & 0.0 & 14.2 & 18.9 & 0.0 \\
\hline 4 & & 4 & 62 & 89.2 & 68.2 & 0.0 & 28.0 & 9.2 & 10.1 & 25.8 & 26.9 & 0.0 \\
\hline 4 & & & 122 & 89.1 & 67.9 & 0.0 & 45.2 & 10.4 & 11.8 & 32.6 & 0.0 & 0.0 \\
\hline 5 & $121-131$ & 1 & 7 & 84.5 & 54.4 & 17.9 & 77.1 & 2.9 & 1.4 & 0.0 & 0.0 & 0.0 \\
\hline 5 & & & 36 & 89.0 & 67.6 & 22.2 & 41.1 & 7.0 & 0.0 & 13.2 & 16.5 & 0.0 \\
\hline \multicolumn{13}{|l|}{ Hole 24} \\
\hline Core & $\begin{array}{l}\text { Depth Below } \\
\text { Sea Floor } \\
\text { (m) }\end{array}$ & Section & $\begin{array}{l}\text { Depth In } \\
\text { Section } \\
(\mathrm{cm})\end{array}$ & Diff. & $\begin{array}{c}\% \\
\text { Amorph. } \\
\text { Scattering }\end{array}$ & Calc. & Quar. & $\mathrm{K}-\mathrm{Fe}$ & Plag. & Kaol. & Mica & Chlor. \\
\hline 1 & $198-209$ & 1 & 33 & 87.5 & 63.2 & 7.3 & 55.9 & 5.6 & 6.7 & 11.1 & 13.4 & 0.0 \\
\hline 2 & $209-216$ & 1 & 20 & 84.4 & 54.1 & 42.7 & 57.3 & 0.0 & 0.0 & 0.0 & 0.0 & 0.0 \\
\hline
\end{tabular}


TABLE 1 - Continued

\begin{tabular}{|c|c|c|c|c|c|c|c|c|c|c|c|c|}
\hline \multicolumn{13}{|c|}{ Hole 24 - Continued } \\
\hline Core & $\begin{array}{l}\text { Depth Below } \\
\text { Sea Floor } \\
\text { (m) }\end{array}$ & Section & $\begin{array}{l}\text { Depth In } \\
\text { Section } \\
(\mathrm{cm})\end{array}$ & Diff. & $\begin{array}{c}\% \\
\text { Amorph. } \\
\text { Scattering }\end{array}$ & Calc. & Quar. & $\mathrm{K}-\mathrm{Fe}$ & Plag. & Kaol. & Mica & Chlor. \\
\hline 4 & $225-234$ & 2 & 8 & 89.5 & 69.1 & 0.0 & 42.4 & 12.7 & 11.0 & 0.0 & 0.0 & 33.9 \\
\hline 4 & & & 91 & 88.9 & 67.4 & 0.0 & 39.5 & 11.8 & 11.8 & 15.8 & 0.0 & 20.9 \\
\hline 4 & & 3 & 22 & 84.0 & 52.9 & 0.0 & 43.9 & 5.4 & 4.0 & 7.0 & 21.2 & 17.8 \\
\hline 4 & & & 83 & 88.3 & 65.6 & 0.0 & 37.0 & 3.3 & 5.1 & 17.2 & 28.5 & 8.8 \\
\hline 4 & & 4 & 9 & 89.0 & 67.6 & 0.0 & 31.2 & 4.8 & 3.8 & 21.3 & 25.1 & 13.9 \\
\hline 4 & & & 91 & 89.2 & 68.2 & 0.0 & 29.6 & 6.2 & 5.9 & 11.8 & 30.8 & 15.7 \\
\hline \multicolumn{13}{|l|}{ Hole 24A } \\
\hline Core & $\begin{array}{l}\text { Depth Below } \\
\text { Sea Floor } \\
\text { (m) }\end{array}$ & Section & $\begin{array}{l}\text { Depth In } \\
\text { Section } \\
\text { (cm) }\end{array}$ & Diff. & $\begin{array}{c}\% \\
\text { Amorph. } \\
\text { Scattering }\end{array}$ & Quar. & $\mathrm{K}-\mathrm{Fe}$ & Plag. & Mica & Pyrite & Cris. & \\
\hline 1 & $503-511$ & 1 & 24 & 89.2 & 68.2 & 7.4 & - & - & - & - & 92.6 & \\
\hline 2 & $511-520$ & 1 & 27 & 89.1 & 67.9 & 10.8 & 5.7 & 4.9 & 15.6 & - & 63.0 & \\
\hline 3 & $540-550$ & 1 & 101 & 88.9 & 67.4 & 11.1 & 4.0 & 5.3 & 11.6 & 1.8 & 66.1 & \\
\hline 4 & $550-558$ & 2 & 60 & 89.2 & 68.2 & 11.1 & 4.7 & 5.7 & 13.4 & 3.8 & 61.3 & \\
\hline \multicolumn{13}{|l|}{ Hole 25} \\
\hline Core & $\begin{array}{l}\text { Depth Below } \\
\text { Sea Floor } \\
\text { (m) }\end{array}$ & Section & $\begin{array}{l}\text { Depth In } \\
\text { Section } \\
\text { (cm) }\end{array}$ & Diff. & $\begin{array}{c}\% \\
\text { Amorph. } \\
\text { Scattering }\end{array}$ & Calc. & Quar. & Kaol. & & & & \\
\hline 1 & $0-9$ & 4 & 110 & 85.5 & 57.4 & 95.3 & 4.7 & 0.0 & & & & \\
\hline 1 & & 5 & 30 & 85.1 & 56.2 & 96.7 & 3.2 & 0.0 & & & & \\
\hline 1 & & 6 & 68 & 85.6 & 57.6 & 96.6 & 3.4 & 0.0 & & & & \\
\hline 2 & $9-18$ & 2 & 30 & 85.1 & 56.2 & 96.8 & 3.2 & 0.0 & & & & \\
\hline 2 & & 3 & 70 & 85.5 & 57.4 & 91.6 & 4.4 & 3.4 & & & & \\
\hline 3 & $18-27$ & 1 & 125 & 84.8 & 55.3 & 96.6 & 3.4 & 0.0 & & & & \\
\hline 3 & & 2 & 38 & 84.6 & 54.7 & 95.6 & 4.4 & 0.0 & & & & \\
\hline
\end{tabular}


TABLE 1 - Continued

\begin{tabular}{|c|c|c|c|c|c|c|c|c|c|c|c|}
\hline \multicolumn{12}{|c|}{ Hole 25 - Continued } \\
\hline Core & $\begin{array}{l}\text { Depth Below } \\
\text { Sea Floor } \\
\text { (m) }\end{array}$ & Section & $\begin{array}{l}\text { Depth In } \\
\text { Section } \\
\text { (cm) }\end{array}$ & Diff. & $\begin{array}{c}\% \\
\text { Amorph. } \\
\text { Scattering }\end{array}$ & Calc. & Quar. & Kaol. & & & \\
\hline 3 & $18-27$ & 3 & 75 & 84.4 & 54.1 & 96.4 & 3.3 & 0.0 & & & \\
\hline 3 & & 4 & 75 & 84.4 & 54.1 & 96.4 & 3.4 & 0.0 & & & \\
\hline 3 & & 5 & 75 & 84.8 & 55.3 & 96.2 & 3.8 & 0.0 & & & \\
\hline 3 & & 6 & 75 & 84.8 & 55.3 & 96.2 & 3.7 & 0.0 & & & \\
\hline 4 & $27-36$ & 1 & 33 & 84.9 & 55.6 & 95.3 & 4.7 & 0.0 & & & \\
\hline 4 & & 2 & 75 & 84.9 & 55.6 & 95.2 & 3.5 & 0.0 & & & \\
\hline 4 & & 3 & 75 & 84.9 & 55.6 & 96.1 & 3.2 & 0.0 & & & \\
\hline 4 & & 4 & 76 & 84.8 & 55.3 & 97.9 & 1.5 & 0.0 & & & \\
\hline \multicolumn{12}{|l|}{ Hole 25A } \\
\hline Core & $\begin{array}{l}\text { Depth Below } \\
\text { Sea Floor } \\
\text { (m) }\end{array}$ & Section & $\begin{array}{l}\text { Depth In } \\
\text { Section } \\
\text { (cm) }\end{array}$ & Diff. & $\begin{array}{c}\% \\
\text { Amorph. } \\
\text { Scattering }\end{array}$ & Calc. & Quar. & & & & \\
\hline 1 & $49-58$ & 1 & 11 & 84.8 & 55.3 & 95.8 & 4.0 & & & & \\
\hline 1 & & & 92 & 84.9 & 55.6 & 98.2 & 1.3 & & & & \\
\hline 1 & & 2 & 75 & 84.9 & 55.6 & 98.7 & 1.3 & & & & \\
\hline \multicolumn{12}{|l|}{ Hole 26} \\
\hline Core & $\begin{array}{l}\text { Depth Below } \\
\text { Sea Floor } \\
\text { (m) }\end{array}$ & Section & $\begin{array}{l}\text { Depth In } \\
\text { Section } \\
\text { (cm) }\end{array}$ & Diff. & $\begin{array}{c}\% \\
\text { Amorph. } \\
\text { Scattering }\end{array}$ & Quar. & $\mathrm{K}-\mathrm{Fe}$ & Plag. & Kaol. & Mica & Chlor. \\
\hline 1 & $96-105$ & 2 & 5 & 87.5 & 63.2 & 39.1 & 2.0 & 6.1 & 13.0 & 33.6 & 6.2 \\
\hline 1 & & & 94 & 87.8 & 64.1 & 34.6 & 5.9 & 7.3 & 9.7 & 33.2 & 9.3 \\
\hline 1 & & 3 & 16 & 87.8 & 64.1 & 32.0 & 3.8 & 6.4 & 9.0 & 38.5 & 10.2 \\
\hline 1 & & & 93 & 87.8 & 64.1 & 33.0 & 1.4 & 3.3 & 12.4 & 40.6 & 9.2 \\
\hline 1 & & & 144 & 84.9 & 55.6 & 61.1 & 5.1 & 10.1 & 0.0 & 18.5 & 4.0 \\
\hline
\end{tabular}


TABLE 1 - Continued

\begin{tabular}{|c|c|c|c|c|c|c|c|c|c|c|c|c|c|c|c|}
\hline \multicolumn{16}{|c|}{ Hole 26 - Continued } \\
\hline Core & $\begin{array}{l}\text { Depth Below } \\
\text { Sea Floor } \\
\text { (m) }\end{array}$ & Section & $\begin{array}{l}\text { Depth In } \\
\text { Section } \\
\text { (cm) }\end{array}$ & Diff. & $\begin{array}{c}\% \\
\text { Amorph. } \\
\text { Scattering }\end{array}$ & Quar. & $\mathrm{K}-\mathrm{Fe}$ & Plag. & Kaol. & Mica & Chlor. & & & & \\
\hline 2 & $105-114$ & 1 & 129 & 87.7 & 63.8 & 31.4 & 5.3 & 6.6 & 8.8 & 37.7 & 10.1 & & & & \\
\hline 2 & & 2 & 15 & 87.8 & 64.1 & 28.8 & 5.2 & 6.6 & 10.4 & 39.2 & 9.8 & & & & \\
\hline 2 & & & 105 & 87.6 & 63.5 & 35.7 & 0.0 & 5.9 & 11.9 & 37.6 & 8.3 & & & & \\
\hline 3 & $227-238$ & 2 & 15 & 87.6 & 63.5 & 26.6 & 0.0 & 6.9 & 14.9 & 42.5 & 9.0 & & & & \\
\hline 3 & & & 90 & 88.2 & 65.3 & 26.8 & 0.0 & 2.6 & 22.2 & 39.3 & 8.4 & & & & \\
\hline 5 & $477-483$ & 1 & 19 & 87.8 & 64.1 & 30.2 & 0.0 & 3.9 & 13.0 & 40.1 & 12.9 & & & & \\
\hline 5 & & & 95 & 87.9 & 64.4 & 28.8 & 0.0 & 3.3 & 18.6 & 37.6 & 10.8 & & & & \\
\hline 5 & & 2 & 30 & 87.9 & 64.4 & 32.5 & 0.0 & 6.5 & 14.3 & 36.4 & 10.4 & & & & \\
\hline 5 & & & 99 & 87.9 & 64.4 & 34.0 & 0.0 & 6.1 & 16.3 & 32.6 & 10.9 & & & & \\
\hline 5 & & 3 & 33 & 87.8 & 64.1 & 30.4 & 0.0 & 6.4 & 14.6 & 36.5 & 12.1 & & & & \\
\hline 5 & & & 112 & 85.1 & 56.2 & 49.7 & 0.0 & 10.0 & 6.0 & 27.8 & 6.5 & & & & \\
\hline 5 & & 4 & 25 & 87.4 & 62.9 & 29.4 & 0.0 & 5.9 & 15.3 & 40.0 & 9.4 & & & & \\
\hline 5 & & & 92 & 87.4 & 62.9 & 32.8 & 0.0 & 5.9 & 11.2 & 38.1 & 10.3 & & & & \\
\hline \multicolumn{16}{|l|}{ Hole 27} \\
\hline Core & $\begin{array}{l}\text { Depth Below } \\
\text { Sea Floor } \\
\text { (m) }\end{array}$ & Section & $\begin{array}{l}\text { Depth In } \\
\text { Section } \\
\text { (cm) }\end{array}$ & Diff. & $\begin{array}{c}\% \\
\text { Amorph. } \\
\text { Scattering }\end{array}$ & Calc. & Quar. & Plag. & Kaol. & Mica & Chlor. & Mont. & Pyrite & Side. & Clin. \\
\hline 1 & $84-93$ & 1 & 25 & 88.2 & 65.3 & 0.0 & 34.8 & 7.3 & 9.8 & 34.1 & 13.9 & 0.0 & 0.0 & 0.0 & 0.0 \\
\hline 1 & & & 80 & 88.4 & 65.9 & 0.0 & 32.9 & 8.7 & 10.0 & 36.2 & 12.2 & 0.0 & 0.0 & 0.0 & 0.0 \\
\hline 1 & & 2 & 53 & 88.5 & 66.2 & 0.0 & 31.8 & 6.0 & 24.3 & 30.8 & 6.5 & 0.0 & 0.0 & 0.0 & 0.0 \\
\hline 1 & & & 109 & 88.2 & 65.3 & 0.0 & 30.6 & 8.2 & 12.2 & 37.6 & 11.3 & 0.0 & 0.0 & 0.0 & 0.0 \\
\hline 1 & & 6 & 37 & 88.2 & 65.3 & 0.0 & 36.1 & 5.7 & 17.8 & 31.4 & 9.0 & 0.0 & 0.0 & 0.0 & 0.0 \\
\hline 1 & & & 76 & 88.3 & 65.6 & 0.0 & 19.2 & 8.1 & 16.2 & 18.8 & 0.0 & 37.7 & 0.0 & 0.0 & 0.0 \\
\hline 2 & $141-150$ & 1 & 90 & 88.4 & 65.9 & 0.0 & 28.8 & 13.0 & 25.4 & 19.0 & 13.8 & 0.0 & 0.0 & 0.0 & 0.0 \\
\hline 2 & & & 131 & 88.5 & 66.2 & 0.0 & 30.3 & 9.1 & 60.6 & 0.0 & 0.0 & 0.0 & 0.0 & 0.0 & 0.0 \\
\hline 2 & & 2 & 17 & 88.1 & 65.0 & 0.0 & 22.1 & 11.7 & 16.8 & 0.0 & 18.1 & 31.3 & 0.0 & 0.0 & 0.0 \\
\hline
\end{tabular}


TABLE 1 - Continued

\begin{tabular}{|c|c|c|c|c|c|c|c|c|c|c|c|c|c|c|c|}
\hline \multicolumn{16}{|c|}{ Hole 27 - Continued } \\
\hline Core & $\begin{array}{l}\text { Depth Below } \\
\text { Sea Floor } \\
\text { (m) }\end{array}$ & Section & $\begin{array}{l}\text { Depth In } \\
\text { Section } \\
\text { (cm) }\end{array}$ & Diff. & $\begin{array}{c}\% \\
\text { Amoriph. } \\
\text { Scattering }\end{array}$ & Calc. & Quar. & Plag. & Kaol. & Mica & Chlor. & Mont. & Pyrite & Side. & Clin. \\
\hline 2 & $141-150$ & 2 & 84 & 88.3 & 65.6 & 0.0 & 36.5 & 15.3 & 48.2 & 0.0 & 0.0 & 0.0 & 0.0 & 0.0 & 0.0 \\
\hline 2 & & 3 & $25-27$ & 88.3 & 65.6 & 0.0 & 28.7 & 6.6 & 19.5 & 33.0 & 12.1 & 0.0 & 0.0 & 0.0 & 0.0 \\
\hline 2 & & & $99-101$ & 88.0 & 64.7 & 0.0 & 26.5 & 7.4 & 25.4 & 21.7 & 19.0 & 0.0 & 0.0 & 0.0 & 0.0 \\
\hline 3 & $235-244$ & 1 & 51 & 88.3 & 65.6 & 0.0 & 40.2 & 6.8 & - & 32.9 & 20.1 & 0.0 & 0.0 & 0.0 & 0.0 \\
\hline 3 & & & 97 & 87.7 & 63.8 & 0.0 & 26.0 & 4.7 & 10.4 & 21.4 & 9.6 & 27.9 & 0.0 & 0.0 & 0.0 \\
\hline 3 & & 2 & 22 & 88.1 & 65.0 & 0.0 & 39.5 & 0.0 & 12.6 & 22.5 & 19.0 & 0.0 & 6.3 & 0.0 & 0.0 \\
\hline 3 & & & 93 & 87.2 & 62.4 & 0.0 & 44.8 & 8.1 & 17.9 & 18.4 & 10.8 & 0.0 & 0.0 & 0.0 & 0.0 \\
\hline 4 & $244-249$ & 1 & 49 & 88.1 & 65.0 & 0.0 & 36.2 & 0.0 & 15.9 & 32.6 & 15.2 & 0.0 & 0.0 & 0.0 & 0.0 \\
\hline 4 & & & 104 & 88.0 & 64.7 & 0.0 & 32.1 & 5.8 & 33.3 & 28.8 & 0.0 & 0.0 & 0.0 & 0.0 & 0.0 \\
\hline 4 & & 2 & 14 & 88.1 & 65.0 & 0.0 & 34.1 & 3.5 & 21.6 & 30.0 & 10.2 & 0.0 & 0.0 & 0.0 & 0.0 \\
\hline 4 & & & 91 & 88.2 & 65.3 & 0.0 & 31.7 & 5.4 & 34.3 & 28.6 & 0.0 & 0.0 & 0.0 & 0.0 & 0.0 \\
\hline 4 & & 3 & $7-8$ & 88.2 & 65.3 & 0.0 & 37.6 & 0.0 & 16.5 & 30.8 & 15.0 & 0.0 & 0.0 & 0.0 & 0.0 \\
\hline 4 & & & 73 & 87.9 & 64.4 & 0.0 & 38.5 & 0.0 & 10.8 & 34.6 & 16.2 & 0.0 & 0.0 & 0.0 & 0.0 \\
\hline 5 & $369-378$ & 1 & 28 & 88.2 & 65.3 & 0.0 & 39.7 & 0.0 & 17.5 & 32.5 & 10.3 & 0.0 & 0.0 & 0.0 & 0.0 \\
\hline 5 & & & 110 & 87.0 & 61.8 & 45.9 & 6.3 & 0.0 & 0.0 & 0.0 & 1.5 & 0.0 & 0.0 & 46.3 & 0.0 \\
\hline 5 & & 2 & 35 & 87.7 & 63.8 & 0.0 & 37.9 & 6.4 & 13.6 & 31.1 & 6.8 & 0.0 & 4.2 & 0.0 & 0.0 \\
\hline 5 & & & 90 & 86.9 & 61.5 & 50.1 & 11.7 & 0.0 & 19.8 & 8.6 & 0.0 & 0.0 & 0.0 & 9.7 & 0.0 \\
\hline 5 & & 3 & 16 & 87.0 & 61.8 & 59.3 & 13.9 & 0.0 & 8.6 & 10.2 & 8.0 & 0.0 & 0.0 & 0.0 & 0.0 \\
\hline 5 & & & 106 & 87.4 & 62.9 & 51.6 & 10.5 & 0.0 & 19.4 & 8.9 & 0.0 & 0.0 & 0.0 & 9.7 & 0.0 \\
\hline 5 & & 4 & 27 & 87.5 & 63.2 & 52.0 & 13.0 & 0.0 & 18.4 & 11.1 & 0.0 & 0.0 & 0.0 & 0.0 & 0.0 \\
\hline 5 & & & 90 & 87.5 & 63.2 & 57.1 & 15.5 & 0.0 & 20.2 & 3.9 & 0.0 & 0.0 & 0.0 & 5.4 & 0.0 \\
\hline 6 & $454-463$ & 2 & $60-70$ & 88.3 & 65.6 & - & 17.6 & - & - & - & 18.6 & 56.2 & - & 3.3 & 7.6 \\
\hline 6 & & 3 & $10-20$ & 88.0 & 64.7 & - & 18.9 & - & 11.3 & - & 12.5 & 50.4 & - & - & 7.0 \\
\hline
\end{tabular}


TABLE 1 - Continued

\begin{tabular}{|c|c|c|c|c|c|c|c|c|c|c|c|c|c|}
\hline \multicolumn{14}{|l|}{ Hole 27A } \\
\hline Core & $\begin{array}{l}\text { Depth Below } \\
\text { Sea Floor } \\
\text { (m) }\end{array}$ & Section & $\begin{array}{l}\text { Depth In } \\
\text { Section } \\
\text { (cm) }\end{array}$ & Diff. & $\begin{array}{c}\% \\
\text { Amorph. } \\
\text { Scattering }\end{array}$ & Quar. & $\mathrm{K}-\mathrm{Fe}$ & Plag. & Kaol. & Mica & Chlor. & Mont. & Clin. \\
\hline 1 & $25-34$ & 1 & $33-35$ & 90.8 & 72.9 & 33.8 & 0.0 & 8.4 & 13.5 & 36.1 & 8.1 & - & - \\
\hline 1 & & & $82-84$ & 90.6 & 72.4 & 29.9 & 0.0 & 7.5 & 12.0 & 29.3 & 5.4 & 15.9 & 0.0 \\
\hline 1 & & 4 & $19-21$ & 90.7 & 72.6 & 28.3 & 0.0 & 7.6 & 12.5 & 27.8 & 3.7 & 20.1 & 0.0 \\
\hline 1 & & & $71-73$ & 90.8 & 72.9 & 27.0 & 0.0 & 7.0 & 11.9 & 26.5 & 3.5 & 24.1 & 0.0 \\
\hline 1 & & 5 & 35 & 91.0 & 73.5 & 29.1 & 0.0 & 9.6 & 10.5 & 28.5 & 7.0 & 15.4 & 0.0 \\
\hline 1 & & & 91 & 90.6 & 72.4 & 29.2 & 0.0 & 8.5 & 12.9 & 24.0 & 4.7 & 20.8 & 0.0 \\
\hline 1 & & 6 & 14 & 90.6 & 72.4 & 26.5 & 0.0 & 7.2 & 6.4 & 26.0 & 5.6 & 28.4 & 0.0 \\
\hline 1 & & & 66 & 91.1 & 73.8 & 34.8 & 0.0 & 11.5 & 22.3 & 31.4 & 0.0 & 0.0 & 0.0 \\
\hline 2 & $45-54$ & 3 & 31 & 90.7 & 72.6 & 25.8 & 0.0 & 6.7 & 7.2 & 31.8 & 5.4 & 23.0 & 0.0 \\
\hline 2 & & & 81 & 89.7 & 69.7 & 41.8 & 0.0 & 10.9 & 8.4 & 20.5 & 3.3 & 15.1 & 0.0 \\
\hline 3 & $54-63$ & 1 & 117 & 90.6 & 72.4 & 33.6 & 0.0 & 14.8 & 18.8 & 32.9 & 0.0 & 0.0 & 0.0 \\
\hline 3 & & 2 & 33 & 90.3 & 71.5 & 33.8 & 0.0 & 8.1 & 12.2 & 41.6 & 4.4 & 0.0 & 0.0 \\
\hline 3 & & 3 & 35 & 89.6 & 69.4 & 34.6 & 0.0 & 6.2 & 11.1 & 42.6 & 5.5 & 0.0 & 0.0 \\
\hline 3 & $?$ & & 98 & 0.0 & 0.0 & 32.9 & 0.0 & 7.9 & 7.9 & 29.6 & 4.3 & 17.4 & 0.0 \\
\hline 4 & $63-72$ & 1 & 31 & 90.7 & 72.6 & 27.2 & 0.0 & 5.2 & 7.6 & 33.5 & 7.1 & 19.3 & 0.0 \\
\hline 4 & & & $90-92$ & 90.7 & 72.6 & 27.2 & 4.9 & 5.7 & 9.8 & 31.3 & 6.5 & 14.4 & 0.0 \\
\hline 4 & & 2 & 35 & 90.1 & 70.9 & 33.0 & 6.0 & 7.9 & 13.2 & 35.4 & 4.3 & 0.0 & 0.0 \\
\hline 4 & & & 109 & 90.5 & 72.1 & 29.8 & 5.1 & 6.8 & 11.9 & 26.8 & 3.9 & 15.8 & 0.0 \\
\hline 4 & & 3 & 25 & 90.5 & 72.1 & 30.6 & 5.2 & 8.3 & 13.5 & 35.2 & 3.4 & 0.0 & 4.0 \\
\hline 4 & & & 72 & 87.4 & 62.9 & 51.3 & 6.2 & 13.9 & 6.2 & 19.0 & 3.6 & 0.0 & 0.0 \\
\hline 4 & & 4 & 26 & 87.1 & 62.1 & 50.0 & 5.5 & 12.5 & 4.0 & 16.5 & 2.5 & 9.0 & 0.0 \\
\hline 4 & & & 105 & 90.4 & 71.8 & 31.5 & 5.7 & 7.6 & 16.4 & 33.8 & 5.0 & 0.0 & 0.0 \\
\hline 4 & & 5 & 30 & 90.6 & 72.4 & 32.1 & 5.8 & 7.7 & 7.7 & 36.9 & 6.7 & 0.0 & 3.2 \\
\hline 4 & & & 80 & 90.4 & 71.8 & 32.6 & 6.2 & 7.5 & 18.2 & 31.9 & 3.6 & 0.0 & 0.0 \\
\hline 4 & & 6 & 29 & 90.5 & 72.1 & 25.9 & 6.5 & 7.0 & 10.4 & 31.9 & 4.7 & 13.7 & 0.0 \\
\hline 4 & & & 141 & 90.5 & 72.1 & 36.4 & - & 8.7 & 17.5 & 32.7 & 4.7 & 0.0 & 0.0 \\
\hline
\end{tabular}


TABLE 1 - Continued

\begin{tabular}{|c|c|c|c|c|c|c|c|c|c|c|c|c|c|}
\hline \multicolumn{14}{|c|}{ Hole 27 A - Continued } \\
\hline Core & $\begin{array}{l}\text { Depth Below } \\
\text { Sea Floor } \\
\text { (m) }\end{array}$ & Section & $\begin{array}{l}\text { Depth In } \\
\text { Section } \\
\text { (cm) }\end{array}$ & Diff. & $\begin{array}{c}\% \\
\text { Amorph. } \\
\text { Scattering }\end{array}$ & Quar. & $\mathrm{K}-\mathrm{Fe}$ & Plag. & Kaol. & Mica & Chlor. & Mont. & Clin. \\
\hline 5 & $72-81$ & 1 & $28-30$ & 90.6 & 72.4 & 26.0 & 8.1 & 8.6 & 10.4 & 27.8 & 5.5 & 13.8 & 0.0 \\
\hline 5 & & 2 & 23 & 90.7 & 72.6 & 36.5 & - & 9.1 & 17.5 & 32.8 & 4.0 & 0.0 & 0.0 \\
\hline 5 & & 3 & 14 & 90.7 & 72.6 & 33.8 & 6.4 & 7.1 & 13.5 & 33.1 & 6.1 & 0.0 & 0.0 \\
\hline 5 & & & 108 & 90.5 & 72.1 & 33.8 & 6.1 & 8.1 & 13.5 & 33.1 & 5.4 & 0.0 & 0.0 \\
\hline 5 & & 4 & 38 & 90.5 & 72.1 & 32.6 & 5.9 & 7.5 & 16.9 & 31.9 & 5.2 & 0.0 & 0.0 \\
\hline 5 & & & 129 & 0.0 & 0.0 & 0.0 & 0.0 & 0.0 & 0.0 & 0.0 & 0.0 & 0.0 & 0.0 \\
\hline 5 & & 5 & 37 & 90.6 & 72.4 & 31.9 & 6.7 & 8.6 & 20.4 & 28.8 & 3.5 & 0.0 & 0.0 \\
\hline 5 & & & 125 & 90.2 & 71.2 & 29.3 & 5.6 & 6.2 & 15.2 & 38.4 & 5.3 & 0.0 & 0.0 \\
\hline \multicolumn{14}{|l|}{ Hole 28} \\
\hline Core & $\begin{array}{l}\text { Depth Below } \\
\text { Sea Floor } \\
\text { (m) }\end{array}$ & Section & $\begin{array}{l}\text { Depth In } \\
\text { Section } \\
\text { (cm) }\end{array}$ & Diff. & $\begin{array}{c}\% \\
\text { Amorph. } \\
\text { Scattering }\end{array}$ & Calc. & Quar. & Plag. & Kaol. & Mica & Chlor. & Mont. & Clin. \\
\hline 2 & $68-77$ & 2 & 16.5 & 89.0 & 67.6 & 0.0 & 44.8 & 25.6 & 0.0 & 0.0 & 29.6 & 0.0 & 0.0 \\
\hline 2 & & & 85.5 & 88.4 & 65.9 & 0.0 & 14.9 & 43.0 & 0.0 & 18.4 & 23.7 & 0.0 & 0.0 \\
\hline 2 & & 3 & 20 & 89.3 & 68.5 & 0.0 & 34.7 & 16.7 & 0.0 & 28.5 & 20.1 & 0.0 & 0.0 \\
\hline 2 & & & 80 & 89.0 & 67.6 & 0.0 & 41.2 & 12.3 & 24.7 & 0.0 & 21.8 & 0.0 & 0.0 \\
\hline 2 & & 4 & 22 & 89.4 & 68.8 & 0.0 & 35.1 & 14.7 & 7.0 & 20.0 & 23.2 & 0.0 & 0.0 \\
\hline 2 & & & 86 & 88.9 & 67.4 & 0.0 & 37.9 & 14.0 & 0.0 & 28.0 & 20.1 & 0.0 & 0.0 \\
\hline 3 & $169-176^{\mathrm{a}}$ & 1 & 20 & 88.4 & 65.9 & 46.6 & 5.3 & 0.0 & 3.2 & 0.0 & 0.0 & 42.3 & 2.6 \\
\hline 3 & & & 93 & 88.0 & 64.7 & 56.1 & 6.2 & 1.4 & 0.0 & 0.0 & 9.9 & 22.2 & 4.2 \\
\hline 3 & & 2 & 44 & 88.0 & 64.7 & 53.3 & 6.6 & 1.5 & 0.0 & 0.0 & 6.2 & 23.6 & 8.8 \\
\hline 3 & & & 80 & 88.2 & 65.3 & 54.4 & 6.0 & 0.0 & 0.0 & 0.0 & 8.0 & 21.5 & 10.1 \\
\hline 3 & & 3 & $35-37$ & 88.1 & 65.0 & 54.8 & 6.2 & 0.0 & 0.0 & 0.0 & 6.6 & 22.1 & 10.4 \\
\hline 3 & & & $121-123$ & 88.3 & 65.6 & 46.2 & 5.4 & 0.0 & 0.0 & 0.0 & 7.1 & 33.3 & 8.0 \\
\hline 3 & & 4 & 51 & 89.0 & 67.6 & 0.0 & 14.5 & 0.0 & 0.0 & 0.0 & 23.3 & 25.9 & 36.4 \\
\hline
\end{tabular}

${ }^{a}$ Note: Plot indicates 9 meters of recovery in order to accommodate the samples received. 
TABLE 1 - Continued

\begin{tabular}{|c|c|c|c|c|c|c|c|c|c|c|c|c|c|c|}
\hline \multicolumn{15}{|c|}{ Hole 28 - Continued } \\
\hline Core & $\begin{array}{l}\text { Depth Below } \\
\text { Sea Floor } \\
\text { (m) }\end{array}$ & Section & $\begin{array}{l}\text { Depth In } \\
\text { Section } \\
\text { (cm) }\end{array}$ & Diff. & $\begin{array}{c}\% \\
\text { Amorph. } \\
\text { Scattering }\end{array}$ & Calc. & Quar. & Plag. & Kaol. & Mica & Chlor. & Mont. & Clin. & \\
\hline 3 & $169-176^{\mathrm{a}}$ & 4 & 71.5 & 87.9 & 64.4 & 51.8 & 4.5 & 0.0 & 0.0 & 0.0 & 4.6 & 39.1 & 0.0 & \\
\hline 3 & & 5 & 18 & 88.1 & 65.0 & 50.9 & 3.6 & 0.0 & 0.0 & 0.0 & 3.7 & 41.7 & 0.0 & \\
\hline 3 & & & 71 & 88.3 & 65.6 & 53.1 & 4.1 & 0.0 & 0.0 & 0.0 & 3.3 & 39.5 & 0.0 & \\
\hline 3 & & 6 & 30 & 87.6 & 63.5 & 77.9 & 6.2 & 0.0 & 0.0 & 0.0 & 15.9 & 0.0 & 0.0 & \\
\hline 3 & & & 124 & 88.6 & 66.5 & 48.0 & 5.8 & 0.0 & 0.0 & 0.0 & 0.0 & 41.4 & 4.8 & \\
\hline \multicolumn{15}{|c|}{${ }^{\mathrm{a}}$ Note: Plot indicates 9 meters of recovery in order to accommodate the samples received. } \\
\hline \multicolumn{15}{|l|}{ Hole 29} \\
\hline Core & $\begin{array}{l}\text { Depth Below } \\
\text { Sea Floor } \\
\text { (m) }\end{array}$ & Section & $\begin{array}{l}\text { Depth In } \\
\text { Section } \\
\text { (cm) }\end{array}$ & Diff. & $\begin{array}{c}\% \\
\text { Amorph. } \\
\text { Scattering }\end{array}$ & Calc. & Quar. & Plag. & Kaol. & Mica & Chlor. & Mont. & Clin. & Sepio. \\
\hline 1 & $0-9$ & 1 & 27 & 87.5 & 63.2 & 67.7 & 12.8 & 2.3 & 7.7 & 9.5 & 0.0 & 0.0 & 0.0 & 0.0 \\
\hline 1 & & & 100 & 87.2 & 62.4 & 68.6 & 11.9 & 2.0 & 3.3 & 9.8 & 4.4 & 0.0 & 0.0 & 0.0 \\
\hline 1 & & 2 & 30 & 87.5 & 63.2 & 60.1 & 14.9 & 1.4 & 11.6 & 11.0 & 0.0 & 0.0 & 0.0 & 0.0 \\
\hline 1 & & & 110 & 87.8 & 64.1 & 57.8 & 17.6 & 1.2 & 7.5 & 11.8 & 4.2 & 0.0 & 0.0 & 0.0 \\
\hline 1 & & 3 & 40 & 87.9 & 64.4 & 42.4 & 18.5 & 3.5 & 11.1 & 19.8 & 4.8 & 0.0 & 0.0 & 0.0 \\
\hline 1 & & & 125 & 87.8 & 64.1 & 48.4 & 18.2 & 3.3 & 5.8 & 17.8 & 6.7 & 0.0 & 0.0 & 0.0 \\
\hline 2 & $9-18$ & 1 & 59 & 87.8 & 64.1 & 52.5 & 18.0 & 3.1 & 9.3 & 11.9 & 5.2 & 0.0 & 0.0 & 0.0 \\
\hline 2 & & & 110 & 87.6 & 63.5 & 61.4 & 15.4 & 3.3 & 5.0 & 10.5 & 4.4 & 0.0 & 0.0 & 0.0 \\
\hline 2 & & 2 & 49.5 & 87.6 & 63.5 & 56.8 & 14.8 & 2.7 & 5.4 & 14.5 & 5.9 & 0.0 & 0.0 & 0.0 \\
\hline 2 & & & $106-109$ & 87.4 & 62.9 & 74.8 & 13.2 & 0.0 & 4.0 & 0.0 & 5.7 & 0.0 & 0.0 & 0.0 \\
\hline 2 & & 3 & 10 & 87.6 & 63.5 & 62.3 & 14.4 & 1.3 & 7.4 & 10.9 & 3.7 & 0.0 & 0.0 & 0.0 \\
\hline 2 & & & 90 & 87.4 & 62.9 & 61.6 & 14.1 & 2.5 & 6.8 & 11.9 & 3.1 & 0.0 & 0.0 & 0.0 \\
\hline 3 & $18-27$ & 1 & 30 & 87.2 & 62.4 & 65.3 & 13.6 & 0.0 & 3.8 & 11.2 & 6.1 & 0.0 & 0.0 & 0.0 \\
\hline 4 & $27-37$ & 1 & $119.5-122$ & 87.5 & 63.2 & 54.5 & 18.0 & 4.1 & 13.9 & 9.6 & 0.0 & 0.0 & 0.0 & 0.0 \\
\hline 4 & & 2 & 49 & 87.6 & 63.5 & 56.7 & 17.7 & 4.2 & 7.5 & 9.9 & 4.0 & 0.0 & 0.0 & 0.0 \\
\hline 4 & & & 95 & 88.2 & 65.3 & 36.1 & 23.4 & 4.6 & 16.7 & 14.6 & 4.6 & 0.0 & 0.0 & 0.0 \\
\hline
\end{tabular}


TABLE 1 - Continued

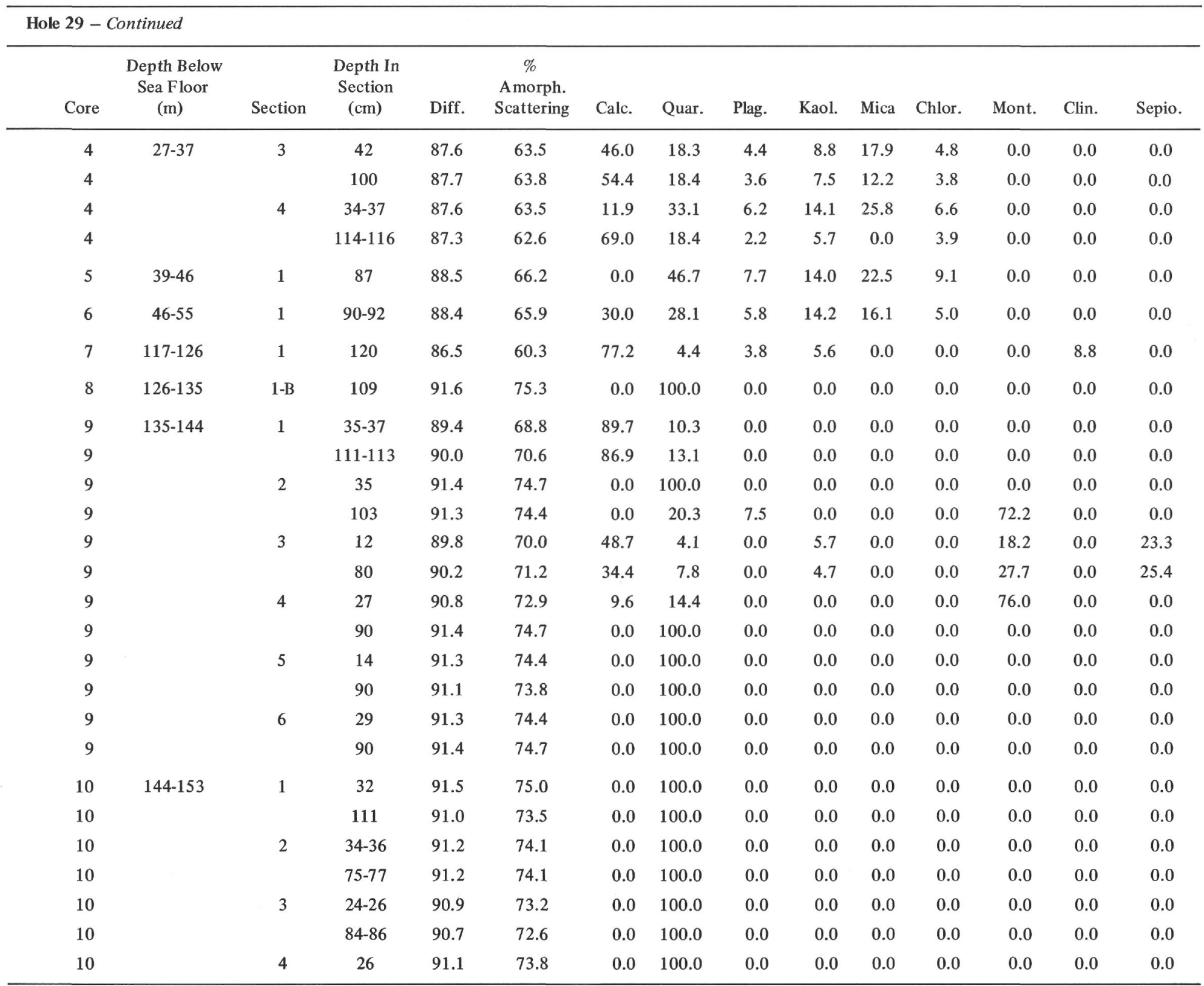


TABLE 1 - Continued

\begin{tabular}{|c|c|c|c|c|c|c|c|c|c|c|c|c|c|c|}
\hline \multicolumn{15}{|c|}{ Hole 29 - Continued } \\
\hline Core & $\begin{array}{l}\text { Depth Below } \\
\text { Sea Floor } \\
\text { (m) }\end{array}$ & Section & $\begin{array}{l}\text { Depth In } \\
\text { Section } \\
(\mathrm{cm})\end{array}$ & Diff. & $\begin{array}{c}\% \\
\text { Amorph. } \\
\text { Scattering }\end{array}$ & Calc. & Quar. & Plag. & Kaol. & Mica & Chlor. & Mont. & Clin. & Sepio. \\
\hline 10 & $144-153$ & 4 & 109 & 91.1 & 73.8 & 0.0 & 100.0 & 0.0 & 0.0 & 0.0 & 0.0 & 0.0 & 0.0 & 0.0 \\
\hline 10 & & 5 & 28 & 91.3 & 74.4 & 0.0 & 100.0 & 0.0 & 0.0 & 0.0 & 0.0 & 0.0 & 0.0 & 0.0 \\
\hline 10 & & & $110-112$ & 91.3 & 74.4 & 0.0 & 100.0 & 0.0 & 0.0 & 0.0 & 0.0 & 0.0 & 0.0 & 0.0 \\
\hline 11 & $153-162$ & 1 & 105 & 91.4 & 74.7 & 0.0 & 100.0 & 0.0 & 0.0 & 0.0 & 0.0 & 0.0 & 0.0 & 0.0 \\
\hline 12 & $162-172$ & 1 & $29-31$ & 91.4 & 74.7 & 0.0 & 100.0 & 0.0 & 0.0 & 0.0 & 0.0 & 0.0 & 0.0 & 0.0 \\
\hline 12 & & & $121-123$ & 91.4 & 74.7 & 0.0 & 100.0 & 0.0 & 0.0 & 0.0 & 0.0 & 0.0 & 0.0 & 0.0 \\
\hline 12 & & 2 & 29 & 91.6 & 75.3 & 0.0 & 100.0 & 0.0 & 0.0 & 0.0 & 0.0 & 0.0 & 0.0 & 0.0 \\
\hline 12 & & & 105 & 91.3 & 74.4 & 0.0 & 100.0 & 0.0 & 0.0 & 0.0 & 0.0 & 0.0 & 0.0 & 0.0 \\
\hline 12 & & 3 & 17 & 90.2 & 71.2 & 88.9 & 11.1 & 0.0 & 0.0 & 0.0 & 0.0 & 0.0 & 0.0 & 0.0 \\
\hline 12 & & & 65 & 90.5 & 72.1 & 88.1 & 11.9 & 0.0 & 0.0 & 0.0 & 0.0 & 0.0 & 0.0 & 0.0 \\
\hline 12 & & 4 & 13 & 88.7 & 66.8 & 97.0 & 3.0 & 0.0 & 0.0 & 0.0 & 0.0 & 0.0 & 0.0 & 0.0 \\
\hline 12 & & & 110 & 89.4 & 68.8 & 95.4 & 4.6 & 0.0 & 0.0 & 0.0 & 0.0 & 0.0 & 0.0 & 0.0 \\
\hline 12 & & 5 & 16 & 88.8 & 67.1 & 95.3 & 3.7 & 0.0 & 0.0 & 0.0 & 0.0 & 0.0 & 0.0 & 0.0 \\
\hline 12 & & & 105 & 88.9 & 67.4 & 97.3 & 2.7 & 0.0 & 0.0 & 0.0 & 0.0 & 0.0 & 0.0 & 0.0 \\
\hline 12 & & 6 & 30 & 89.9 & 70.3 & 96.0 & 4.0 & 0.0 & 0.0 & 0.0 & 0.0 & 0.0 & 0.0 & 0.0 \\
\hline 12 & & & 92 & 89.1 & 67.9 & 95.4 & 4.2 & 0.0 & 0.0 & 0.0 & 0.0 & 0.0 & 0.0 & 0.0 \\
\hline \multirow[t]{2}{*}{14} & $189-193$ & 1 & 25 & 91.4 & 74.7 & 0.0 & 100.0 & 0.0 & 0.0 & 0.0 & 0.0 & 0.0 & 0.0 & 0.0 \\
\hline & & & 72 & 91.5 & 75.0 & 0.0 & 100.0 & 0.0 & 0.0 & 0.0 & 0.0 & 0.0 & 0.0 & 0.0 \\
\hline 14 & & 2 & 31 & 91.3 & 74.4 & 0.0 & 100.0 & 0.0 & 0.0 & 0.0 & 0.0 & 0.0 & 0.0 & 0.0 \\
\hline 14 & & & 82 & 91.5 & 75.0 & 0.0 & 100.0 & 0.0 & 0.0 & 0.0 & 0.0 & 0.0 & 0.0 & 0.0 \\
\hline 14 & & 3 & 25 & 91.5 & 75.0 & 0.0 & 100.0 & 0.0 & 0.0 & 0.0 & 0.0 & 0.0 & 0.0 & 0.0 \\
\hline 14 & & & 81 & 91.3 & 74.4 & 0.0 & 100.0 & 0.0 & 0.0 & 0.0 & 0.0 & 0.0 & 0.0 & 0.0 \\
\hline 14 & & 4 & 25 & 91.3 & 74.4 & 0.0 & 100.0 & 0.0 & 0.0 & 0.0 & 0.0 & 0.0 & 0.0 & 0.0 \\
\hline 14 & & & 100 & 91.3 & 74.4 & 0.0 & 100.0 & 0.0 & 0.0 & 0.0 & 0.0 & 0.0 & 0.0 & 0.0 \\
\hline 14 & & 5 & $24-26$ & 91.4 & 74.7 & 0.0 & 100.0 & 0.0 & 0.0 & 0.0 & 0.0 & 0.0 & 0.0 & 0.0 \\
\hline 14 & & & $74-76$ & 0.0 & 100.0 & 0.0 & - & 0.0 & 0.0 & 0.0 & 0.0 & 0.0 & 0.0 & 0.0 \\
\hline 14 & & 6 & 25 & 93.3 & 80.3 & 0.0 & 100.0 & 0.0 & 0.0 & 0.0 & 0.0 & 0.0 & 0.0 & 0.0 \\
\hline 14 & & & 75 & 91.2 & 74.1 & 0.0 & 100.0 & 0.0 & 0.0 & 0.0 & 0.0 & 0.0 & 0.0 & 0.0 \\
\hline
\end{tabular}


TABLE 1 - Continued

\begin{tabular}{|c|c|c|c|c|c|c|c|c|c|c|c|c|c|c|}
\hline Core & $\begin{array}{l}\text { Depth Below } \\
\text { Sea F loor } \\
\text { (m) }\end{array}$ & Section & $\begin{array}{l}\text { Depth In } \\
\text { Section } \\
(\mathrm{cm})\end{array}$ & Diff. & $\begin{array}{c}\% \\
\text { Amorph. } \\
\text { Scattering }\end{array}$ & Calc. & Quar. & Plag. & Kaol. & Mica. & Chlor. & Mont. & Clin. & Sepio. \\
\hline 15 & 193-202 & 1 & 27 & 91.1 & 73.8 & 22.9 & 2.6 & 0.0 & 0.0 & 0.0 & 0.0 & 32.3 & 0.0 & 42.2 \\
\hline 15 & & & 82 & 91.1 & 73.8 & 92.7 & 7.3 & 0.0 & 0.0 & 0.0 & 0.0 & 0.0 & 0.0 & 0.0 \\
\hline 15 & & 2 & 26 & 90.4 & 71.8 & 30.5 & 2.7 & 0.0 & 0.0 & 0.0 & 0.0 & 27.2 & 0.0 & 39.6 \\
\hline 15 & & & 82 & 91.3 & 74.4 & 0.0 & 100.0 & 0.0 & 0.0 & 0.0 & 0.0 & 0.0 & 0.0 & 0.0 \\
\hline 15 & & 3 & 29 & 91.4 & 74.7 & 0.0 & 100.0 & 0.0 & 0.0 & 0.0 & 0.0 & 0.0 & 0.0 & 0.0 \\
\hline 15 & & & 72 & 91.2 & 74.1 & 0.0 & 100.0 & 0.0 & 0.0 & 0.0 & 0.0 & 0.0 & 0.0 & 0.0 \\
\hline 15 & & 4 & 24 & 91.4 & 74.7 & 49.1 & 50.9 & 0.0 & 0.0 & 0.0 & 0.0 & 0.0 & 0.0 & 0.0 \\
\hline 15 & & & $73-75$ & 91.0 & 73.5 & 9.1 & 3.6 & 0.0 & 0.0 & 0.0 & 0.0 & 51.7 & 0.0 & 35.5 \\
\hline 15 & & 5 & 22 & 91.4 & 74.7 & 0.0 & 100.0 & 0.0 & 0.0 & 0.0 & 0.0 & 0.0 & 0.0 & 0.0 \\
\hline 15 & & & 82 & 91.0 & 73.5 & 4.4 & - & 0.0 & 0.0 & 0.0 & 0.0 & 61.4 & 0.0 & 33.8 \\
\hline 15 & & 6 & 26 & 90.3 & 71.5 & 95.7 & 4.3 & 0.0 & 0.0 & 0.0 & 0.0 & 0.0 & 0.0 & 0.0 \\
\hline 15 & & & 81 & 89.6 & 69.4 & 98.3 & 1.4 & 0.0 & 0.0 & 0.0 & 0.0 & 0.0 & 0.0 & 0.0 \\
\hline 16 & $202-211$ & 1 & 37 & 90.2 & 71.2 & 43.8 & 2.3 & 0.0 & 0.0 & 0.0 & 0.0 & 20.3 & 0.0 & 33.6 \\
\hline 16 & & & 91 & 90.0 & 70.6 & 44.8 & 1.6 & 0.0 & 0.0 & 0.0 & 3.4 & 26.1 & 0.0 & 24.0 \\
\hline 16 & & 2 & 28 & 89.5 & 69.1 & 98.6 & 1.4 & 0.0 & 0.0 & 0.0 & 0.0 & 0.0 & 0.0 & 0.0 \\
\hline 16 & & & 81 & 0.0 & 100.0 & 0.0 & - & 0.0 & 0.0 & 0.0 & 0.0 & 0.0 & 0.0 & 0.0 \\
\hline 16 & & 3 & 38 & 91.2 & 74.1 & 82.7 & 17.3 & 0.0 & 0.0 & 0.0 & 0.0 & 0.0 & 0.0 & 0.0 \\
\hline 16 & & & 81 & 90.8 & 72.9 & 90.6 & 9.1 & 0.0 & 0.0 & 0.0 & 0.0 & 0.0 & 0.0 & 0.0 \\
\hline 16 & & 4 & 31 & 90.9 & 73.2 & 22.8 & 2.2 & 0.0 & 0.0 & 0.0 & 0.0 & 50.2 & 0.0 & 24.8 \\
\hline 16 & & & 72 & 90.6 & 72.4 & 93.2 & 6.8 & 0.0 & 0.0 & 0.0 & 0.0 & 0.0 & 0.0 & 0.0 \\
\hline 16 & & 5 & 26 & 90.8 & 72.9 & 96.1 & 3.9 & 0.0 & 0.0 & 0.0 & 0.0 & 0.0 & 0.0 & 0.0 \\
\hline 16 & & & 78 & 90.7 & 72.6 & 95.6 & 4.4 & 0.0 & 0.0 & 0.0 & 0.0 & 0.0 & 0.0 & 0.0 \\
\hline 16 & & 6 & 26 & 90.6 & 72.4 & 92.7 & 7.3 & 0.0 & 0.0 & 0.0 & 0.0 & 0.0 & 0.0 & 0.0 \\
\hline 16 & & & 73 & 90.6 & 72.4 & 29.1 & 2.4 & 0.0 & 0.0 & 0.0 & 0.0 & 37.8 & 0.0 & 30.7 \\
\hline 17 & $211-220$ & 1 & 26 & 91.7 & 75.6 & 0.0 & 100.0 & 0.0 & 0.0 & 0.0 & 0.0 & 0.0 & 0.0 & 0.0 \\
\hline 17 & & & 71 & 91.6 & 75.3 & 0.0 & 100.0 & 0.0 & 0.0 & 0.0 & 0.0 & 0.0 & 0.0 & 0.0 \\
\hline 17 & & 2 & 26 & 91.7 & 75.6 & 0.0 & 100.0 & 0.0 & 0.0 & 0.0 & 0.0 & 0.0 & 0.0 & 0.0 \\
\hline 17 & & & 82 & 91.3 & 74.4 & 0.0 & 100.0 & 0.0 & 0.0 & 0.0 & 0.0 & 0.0 & 0.0 & 0.0 \\
\hline
\end{tabular}


TABLE 1 - Continued

\begin{tabular}{|c|c|c|c|c|c|c|c|c|c|c|c|c|c|c|c|c|}
\hline \multicolumn{17}{|c|}{ Hole 29 - Continued } \\
\hline Core & $\begin{array}{l}\text { Depth Below } \\
\text { Sea Floor } \\
\text { (m) }\end{array}$ & Section & $\begin{array}{l}\text { Depth In } \\
\text { Section } \\
\text { (cm) }\end{array}$ & Diff. & $\begin{array}{c}\% \\
\text { Amorph. } \\
\text { Scattering }\end{array}$ & Calc. & Quar. & Plag. & Kaol. & Mica & Chlor. & Mont. & Clin. & Sepio. & & \\
\hline 17 & $211-220$ & 3 & 27 & 91.8 & 75.9 & 0.0 & 100.0 & 0.0 & 0.0 & 0.0 & 0.0 & 0.0 & 0.0 & 0.0 & & \\
\hline 17 & & & 82 & 91.6 & 75.3 & 0.0 & 100.0 & 0.0 & 0.0 & 0.0 & 0.0 & 0.0 & 0.0 & 0.0 & & \\
\hline 17 & & 4 & 27 & 91.8 & 75.9 & 0.0 & 100.0 & 0.0 & 0.0 & 0.0 & 0.0 & 0.0 & 0.0 & 0.0 & & \\
\hline 17 & & & 82 & 91.4 & 74.7 & 0.0 & 100.0 & 0.0 & 0.0 & 0.0 & 0.0 & 0.0 & 0.0 & 0.0 & & \\
\hline 17 & & 5 & 27 & 91.5 & 75.0 & 0.0 & 100.0 & 0.0 & 0.0 & 0.0 & 0.0 & 0.0 & 0.0 & 0.0 & & \\
\hline 17 & & & 92 & 91.4 & 74.7 & 0.0 & 100.0 & 0.0 & 0.0 & 0.0 & 0.0 & 0.0 & 0.0 & 0.0 & & \\
\hline 17 & & 6 & 26 & 91.5 & 75.0 & 0.0 & 100.0 & 0.0 & 0.0 & 0.0 & 0.0 & 0.0 & 0.0 & 0.0 & & \\
\hline 17 & & & 82 & 91.7 & 75.6 & 0.0 & 100.0 & 0.0 & 0.0 & 0.0 & 0.0 & 0.0 & 0.0 & 0.0 & & \\
\hline 18 & $220-225$ & 2 & 35 & 91.6 & 75.3 & 0.0 & 100.0 & 0.0 & 0.0 & 0.0 & 0.0 & 0.0 & 0.0 & 0.0 & & \\
\hline 18 & & & 102 & 91.6 & 75.3 & 0.0 & 100.0 & 0.0 & 0.0 & 0.0 & 0.0 & 0.0 & 0.0 & 0.0 & & \\
\hline \multicolumn{17}{|l|}{ Hole 29A } \\
\hline Core & $\begin{array}{l}\text { Depth Below } \\
\text { Sea Floor } \\
\text { (m) }\end{array}$ & Section & $\begin{array}{l}\text { Depth In } \\
\text { Section } \\
\text { (cm) }\end{array}$ & Diff. & $\begin{array}{c}\% \\
\text { Amorph. } \\
\text { Scattering }\end{array}$ & Calc. & Quar. & Plag. & Kaol. & Mica & Chlor. & & & & & \\
\hline 2 & $50-59$ & 1 & 34 & 89.4 & 68.8 & 0.0 & 39.8 & 9.1 & 12.7 & 26.8 & 12.1 & & & & & \\
\hline 2 & & & 98 & 88.9 & 67.4 & 0.0 & 43.8 & 6.3 & 19.6 & 22.3 & 8.0 & & & & & \\
\hline 4 & $68-79$ & 1 & 36 & 87.8 & 64.1 & 71.7 & 16.2 & 3.0 & 4.2 & 0.0 & 4.9 & & & & & \\
\hline 5 & $79-86$ & 1 & 50 & 89.2 & 68.2 & 0.0 & 63.4 & 13.4 & 0.0 & 0.0 & 23.2 & & & & & \\
\hline \multicolumn{17}{|l|}{ Hole 29B } \\
\hline Core & $\begin{array}{l}\text { Depth Below } \\
\text { Sea Floor } \\
\text { (m) }\end{array}$ & Section & $\begin{array}{l}\text { Depth In } \\
\text { Section } \\
(\mathrm{cm})\end{array}$ & Diff. & $\begin{array}{c}\% \\
\text { Amorph. } \\
\text { Scattering }\end{array}$ & Calc. & Dolo. & Quart & $\mathrm{K}-\mathrm{Fe}$ & Plag. & Kaol. & Mica & Chlor. & Mont. & Clin. & Sepio. \\
\hline 1 & $57-66$ & 1 & 17 & 88.6 & 66.5 & 0.0 & 0.0 & 35.6 & 6.8 & 7.8 & 14.2 & 26.3 & 9.3 & 0.0 & 0.0 & 0.0 \\
\hline 1 & & & 92 & 89.0 & 67.6 & 0.0 & 0.0 & 45.4 & 0.0 & 7.2 & 14.0 & 23.1 & 10.2 & 0.0 & 0.0 & 0.0 \\
\hline 1 & & 4 & 125 & 89.1 & 67.9 & 0.0 & 0.0 & 42.7 & 0.0 & 10.3 & 6.8 & 24.4 & 15.8 & 0.0 & 0.0 & 0.0 \\
\hline 1 & & 5 & 31 & 89.2 & 68.2 & 0.0 & 0.0 & 38.8 & 0.0 & 7.6 & 26.8 & 26.7 & 0.0 & 0.0 & 0.0 & 0.0 \\
\hline
\end{tabular}


TABLE 1 - Continued

\begin{tabular}{|c|c|c|c|c|c|c|c|c|c|c|c|c|c|c|c|c|}
\hline \multicolumn{17}{|c|}{ Hole 29B - Continued } \\
\hline Core & $\begin{array}{l}\text { Depth Below } \\
\text { Sea Floor } \\
\text { (m) }\end{array}$ & Section & $\begin{array}{l}\text { Depth In } \\
\text { Section } \\
(\mathrm{cm})\end{array}$ & Diff. & $\begin{array}{c}\% \\
\text { Amorph. } \\
\text { Scattering }\end{array}$ & Calc. & Dolo. & Quar. & $\mathrm{K}-\mathrm{Fe}$ & Plag. & Kaol. & Mica & Chlor. & Mont. & Clin. & Sepio. \\
\hline 1 & $57-66$ & 5 & 74 & 89.2 & 68.2 & 0.0 & 0.0 & 47.0 & 0.0 & 7.0 & 12.3 & 22.1 & 11.6 & 0.0 & 0.0 & 0.0 \\
\hline 1 & & 6 & 27 & 89.0 & 67.6 & 0.0 & 0.0 & 34.1 & 7.2 & 7.5 & 15.0 & 28.0 & 8.2 & 0.0 & 0.0 & 0.0 \\
\hline 1 & & & 90 & 89.2 & 68.2 & 0.0 & 0.0 & 28.0 & 6.2 & 7.3 & 11.2 & 23.0 & 9.5 & 14.8 & 0.0 & 0.0 \\
\hline 2 & $68-77$ & 2 & 31 & 89.3 & 68.5 & 0.0 & 0.0 & 36.4 & 5.1 & 10.9 & 7.3 & 26.9 & 13.4 & 0.0 & 0.0 & 0.0 \\
\hline 2 & & & 92 & 89.0 & 67.6 & 0.0 & 0.0 & 27.5 & 0.0 & 13.2 & 3.3 & 15.6 & 11.0 & 29.4 & 0.0 & 0.0 \\
\hline 2 & & 3 & 12 & 88.5 & 66.2 & 59.0 & 0.0 & 19.3 & 4.3 & 5.6 & 1.5 & 0.0 & 10.3 & 0.0 & 0.0 & 0.0 \\
\hline 2 & & & 82 & 89.4 & 68.8 & 0.0 & 0.0 & 41.8 & 0.0 & 12.6 & 8.4 & 20.5 & 16.7 & 0.0 & 0.0 & 0.0 \\
\hline 2 & & 4 & 12 & 89.2 & 68.2 & 0.0 & 0.0 & 40.0 & 0.0 & 13.6 & 6.4 & 26.4 & 13.6 & 0.0 & 0.0 & 0.0 \\
\hline 2 & & & 87 & 87.4 & 62.9 & 72.2 & 0.0 & 10.6 & 0.0 & 2.9 & 0.0 & 8.7 & 5.6 & 0.0 & 0.0 & 0.0 \\
\hline 3 & $78-87$ & 1 & 36 & 88.9 & 67.4 & 26.3 & 0.0 & 20.4 & 0.0 & 8.2 & 3.3 & 0.0 & 9.2 & 32.6 & 0.0 & 0.0 \\
\hline 3 & & & 104 & 89.2 & 68.2 & 0.0 & 0.0 & 25.1 & 0.0 & 13.5 & 0.0 & 16.5 & 18.0 & 26.8 & 0.0 & 0.0 \\
\hline 3 & & 2 & 92 & 89.1 & 67.9 & 0.0 & 0.0 & 58.9 & 0.0 & 13.7 & 9.8 & 0.0 & 17.7 & 0.0 & 0.0 & 0.0 \\
\hline 4 & $87-97$ & 1 & 21 & 89.1 & 67.9 & 0.0 & 0.0 & 30.6 & 0.0 & 9.2 & 6.1 & 20.2 & 12.2 & 21.7 & 0.0 & 0.0 \\
\hline 4 & & & 72 & 88.9 & 67.4 & 0.0 & 0.0 & 39.1 & 0.0 & 11.3 & 9.4 & 25.8 & 14.4 & 0.0 & 0.0 & 0.0 \\
\hline 4 & & 2 & 22 & 89.4 & 68.8 & 0.0 & 0.0 & 39.8 & 0.0 & 11.6 & 8.0 & 22.7 & 17.9 & 0.0 & 0.0 & 0.0 \\
\hline 4 & & & 101 & 89.0 & 67.6 & 0.0 & 0.0 & 38.6 & 0.0 & 10.0 & 13.9 & 22.0 & 15.4 & 0.0 & 0.0 & 0.0 \\
\hline 4 & & 3 & 26 & 89.5 & 69.1 & 0.0 & 0.0 & 39.4 & 0.0 & 17.7 & 11.0 & 16.1 & 15.7 & 0.0 & 0.0 & 0.0 \\
\hline 4 & & & 82 & 89.2 & 68.2 & 0.0 & 0.0 & 40.0 & 0.0 & 18.0 & 3.2 & 19.6 & 19.2 & 0.0 & 0.0 & 0.0 \\
\hline 4 & & 4 & 22 & 89.4 & 68.8 & 0.0 & 0.0 & 40.0 & 0.0 & 13.2 & 8.0 & 22.8 & 16.0 & 0.0 & 0.0 & 00. \\
\hline 4 & & & 92 & 88.0 & 64.7 & 73.8 & 0.0 & 9.6 & 0.0 & 6.5 & 3.8 & 0.0 & 6.3 & 0.0 & 0.0 & 0.0 \\
\hline 5 & $97-106$ & 1 & 21 & 89.8 & 70.0 & 0.0 & 0.0 & 36.6 & 0.0 & 10.6 & 7.3 & 30.0 & 15.4 & 0.0 & 0.0 & 0.0 \\
\hline 5 & & & 94 & 87.0 & 61.8 & 92.8 & 0.0 & 3.5 & 0.0 & 2.7 & 0.0 & 0.0 & 0.0 & 0.0 & 0.0 & 0.0 \\
\hline 5 & & 2 & 22 & 86.9 & 61.5 & 95.5 & 0.0 & 2.6 & 0.0 & 1.3 & 0.0 & 0.0 & 0.0 & 0.0 & 0.0 & 0.0 \\
\hline 5 & & & 92 & 86.6 & 60.6 & 93.8 & 0.0 & 2.9 & 0.0 & 3.2 & 0.0 & 0.0 & 0.0 & 0.0 & 0.0 & 0.0 \\
\hline 5 & & 3 & 21 & 87.6 & 63.5 & 88.7 & 0.0 & 5.1 & 0.0 & 6.2 & 0.0 & 0.0 & 0.0 & 0.0 & 0.0 & 0.0 \\
\hline 5 & & & 86 & 88.8 & 67.1 & 39.5 & 0.0 & 25.6 & 0.0 & 4.9 & 0.0 & 14.6 & 9.5 & 0.0 & 5.9 & 0.0 \\
\hline 5 & & 4 & 21 & 89.0 & 67.6 & 46.2 & 0.0 & 21.1 & 0.0 & 7.6 & 0.0 & 15.6 & 9.5 & 0.0 & 0.0 & 0.0 \\
\hline
\end{tabular}


TABLE 1 - Continued

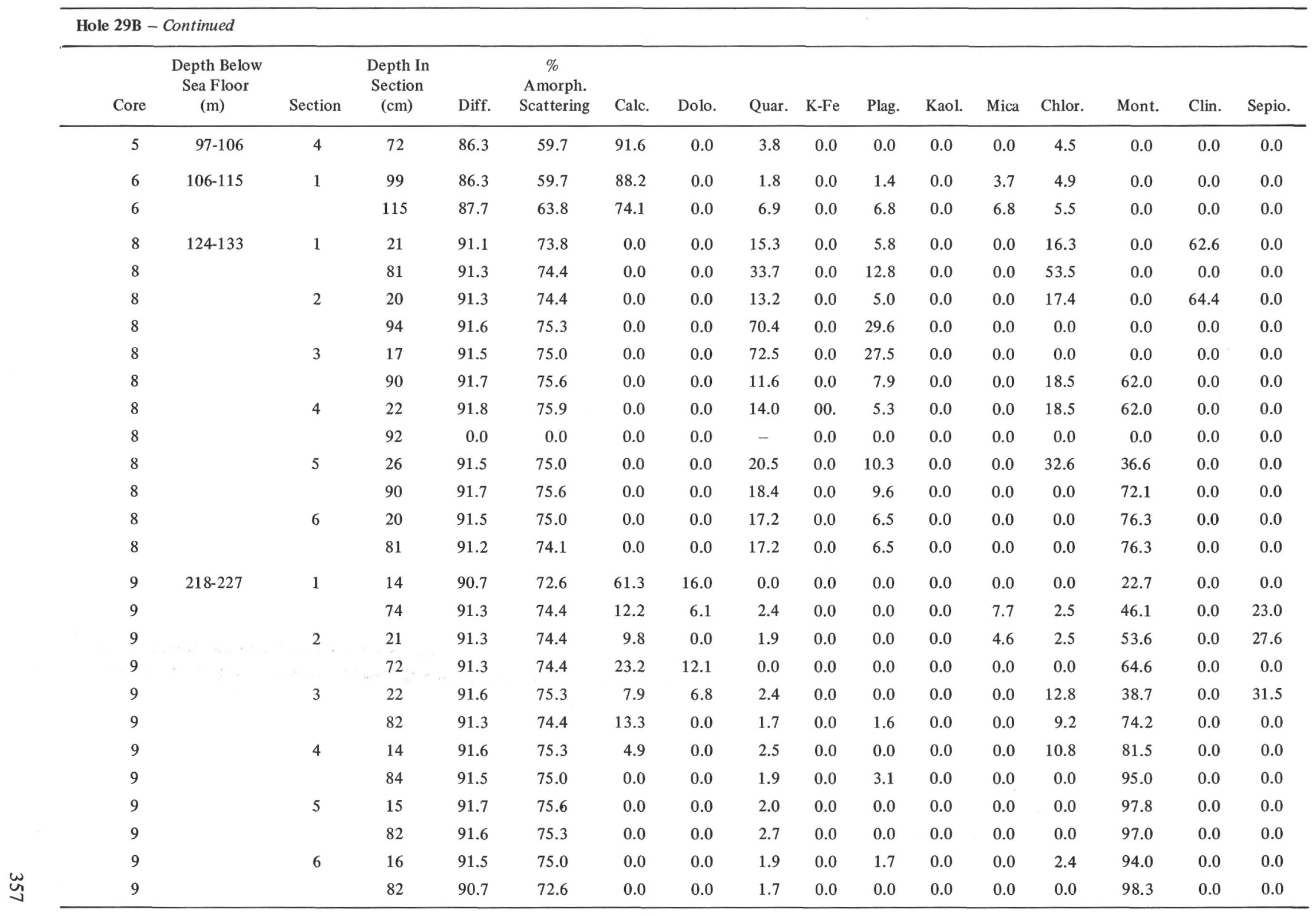


TABLE 1 - Continued

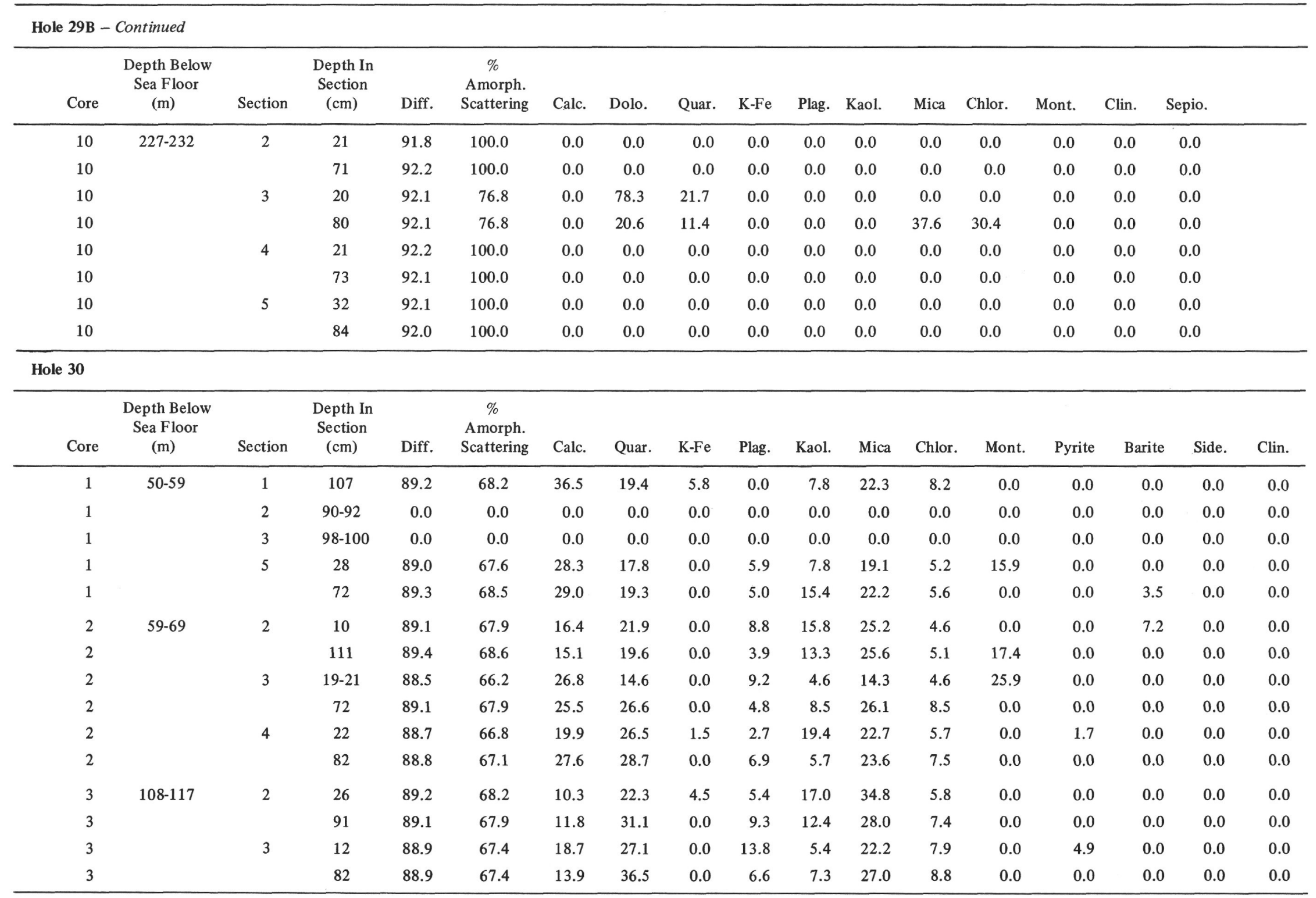


TABLE 1 - Continued

\begin{tabular}{|c|c|c|c|c|c|c|c|c|c|c|c|c|c|c|c|c|c|}
\hline \multicolumn{18}{|c|}{ Hole 30 - Continued } \\
\hline Core & $\begin{array}{l}\text { Depth Below } \\
\text { Sea Floor } \\
\text { (m) }\end{array}$ & Section & $\begin{array}{l}\text { Depth In } \\
\text { Section } \\
\text { (cm) }\end{array}$ & Diff. & $\begin{array}{c}\% \\
\text { Amorph. } \\
\text { Scattering }\end{array}$ & Calc. & Quar. & $\mathrm{K}-\mathrm{Fe}$ & Plag. & Kaol. & Mica & Chlor. & Mont. & Pyrite & Barite & Side. & Clin. \\
\hline 3 & $108-117$ & 4 & 16 & 89.2 & 68.2 & 8.8 & 30.3 & 0.0 & 4.8 & 14.5 & 22.4 & 4.8 & 10.9 & 3.3 & 0.0 & 0.0 & 0.0 \\
\hline 3 & & & 82 & 89.2 & 68.2 & 9.7 & 29.4 & 0.0 & 5.9 & 16.5 & 28.8 & 7.1 & 0.0 & 0.0 & 0.0 & 2.6 & 0.0 \\
\hline 4 & $117-126$ & 1 & 120 & 89.0 & 67.6 & 20.2 & 29.2 & 0.0 & 7.0 & 14.0 & 26.3 & 3.2 & 0.0 & 0.0 & 0.0 & 0.0 & 0.0 \\
\hline 4 & & 2 & 12 & 88.9 & 67.4 & 13.7 & 26.3 & 0.0 & 6.3 & 12.6 & 23.7 & 3.4 & 13.9 & 0.0 & 0.0 & 0.0 & 0.0 \\
\hline 4 & & & 92 & 89.3 & 68.5 & 14.6 & 22.5 & 0.0 & 4.7 & 7.2 & 25.9 & 9.0 & 16.0 & 0.0 & 0.0 & 0.0 & 0.0 \\
\hline 5 & $164-174$ & 1 & 37 & 89.2 & 68.2 & 23.7 & 24.2 & 0.0 & 4.8 & 17.4 & 19.8 & 3.9 & 0.0 & 3.9 & 0.0 & 2.4 & 0.0 \\
\hline 5 & & & 91 & 89.4 & 68.8 & 25.4 & 25.4 & 0.0 & 7.1 & 11.2 & 20.8 & 8.1 & 0.0 & 0.0 & 0.0 & 2.0 & 0.0 \\
\hline 5 & & 2 & 23 & 88.9 & 67.4 & 27.7 & 26.1 & 0.0 & 4.7 & 12.5 & 21.4 & 5.5 & 0.0 & 0.0 & 0.0 & 2.1 & 0.0 \\
\hline 5 & & & 80 & 88.9 & 67.4 & 20.2 & 29.3 & 0.0 & 4.4 & 12.9 & 24.0 & 6.2 & 0.0 & 0.0 & 0.0 & 2.9 & 0.0 \\
\hline 6 & $174-178$ & 1 & 26 & 89.1 & 67.9 & 19.0 & 31.1 & 0.0 & 2.4 & 11.0 & 24.8 & 8.4 & 0.0 & 0.0 & 0.0 & 2.3 & 0.0 \\
\hline 6 & & & 75 & 89.0 & 67.6 & 17.9 & 34.4 & 0.0 & 4.8 & 13.7 & 19.6 & 6.5 & 0.0 & 0.0 & 0.0 & 3.1 & 0.0 \\
\hline 6 & & 2 & 22 & 89.3 & 68.5 & 8.8 & 35.1 & 0.0 & 5.3 & 12.6 & 26.0 & 9.1 & 0.0 & 0.0 & 0.0 & 3.2 & 0.0 \\
\hline 6 & & & 77 & 89.2 & 68.2 & 8.1 & 38.5 & 0.0 & 1.9 & 21.3 & 17.8 & 8.0 & 0.0 & 0.0 & 0.0 & 4.5 & 0.0 \\
\hline 6 & & 3 & 14 & 89.4 & 68.8 & 8.7 & 24.8 & 0.0 & 4.5 & 0.0 & 20.3 & 7.9 & 31.0 & 0.0 & 0.0 & 2.7 & 0.0 \\
\hline 6 & & & 82 & 89.2 & 68.2 & 8.2 & 36.0 & 0.0 & 2.5 & 18.6 & 21.0 & 7.5 & 0.0 & 0.0 & 0.0 & 6.0 & 0.0 \\
\hline 7 & $260-269$ & 1 & 53 & 89.0 & 67.6 & 8.4 & 33.6 & 0.0 & 4.7 & 16.1 & 27.5 & 7.0 & 0.0 & 0.0 & 0.0 & 2.7 & 0.0 \\
\hline 7 & & & 121 & 89.1 & 67.9 & 15.4 & 29.6 & 0.0 & 6.5 & 14.2 & 24.3 & 7.7 & 0.0 & 0.0 & 0.0 & 2.4 & 0.0 \\
\hline 9 & $366-375$ & 1 & 33 & 88.4 & 65.9 & 49.8 & 10.4 & 15.3 & 13.1 & 4.6 & 6.8 & 0.0 & 0.0 & 0.0 & 0.0 & 0.0 & 0.0 \\
\hline 9 & & 2 & 33 & 88.3 & 65.6 & 57.1 & 7.4 & 19.2 & 16.3 & 0.0 & 0.0 & 0.0 & 0.0 & 0.0 & 0.0 & 0.0 & 0.0 \\
\hline 9 & & & 74 & 88.9 & 67.4 & 44.0 & 9.2 & 25.0 & 21.8 & 0.0 & 0.0 & 0.0 & 0.0 & 0.0 & 0.0 & 0.0 & 0.0 \\
\hline 10 & $375-384$ & 1 & 28 & 88.5 & 66.2 & 45.4 & 6.7 & 25.0 & 23.0 & 0.0 & 0.0 & 0.0 & 0.0 & 0.0 & 0.0 & 0.0 & 0.0 \\
\hline 10 & & 2 & 22 & 91.4 & 74.7 & 48.6 & 7.7 & 23.0 & 20.7 & 0.0 & 0.0 & 0.0 & 0.0 & 0.0 & 0.0 & 0.0 & 0.0 \\
\hline 10 & & & 67 & 88.3 & 65.6 & 53.4 & 7.0 & 20.9 & 18.8 & 0.0 & 0.0 & 0.0 & 0.0 & 0.0 & 0.0 & 0.0 & 0.0 \\
\hline 10 & & 3 & 28 & 90.0 & 70.6 & 19.2 & 18.1 & 20.1 & 16.8 & 10.9 & 14.9 & 0.0 & 0.0 & 0.0 & 0.0 & 0.0 & 0.0 \\
\hline 10 & & & 73 & 88.5 & 66.2 & 52.1 & 8.2 & 16.0 & 13.6 & 0.0 & 10.1 & 0.0 & 0.0 & 0.0 & 0.0 & 0.0 & 0.0 \\
\hline
\end{tabular}


TABLE 1 - Continued

\begin{tabular}{|c|c|c|c|c|c|c|c|c|c|c|c|c|c|c|c|c|c|}
\hline \multicolumn{18}{|c|}{ Hole 30 - Continued } \\
\hline Core & $\begin{array}{l}\text { Depth Below } \\
\text { Sea Floor } \\
\text { (m) }\end{array}$ & Section & $\begin{array}{l}\text { Depth In } \\
\text { Section } \\
\text { (cm) }\end{array}$ & Diff. & $\begin{array}{c}\% \\
\text { Amorph. } \\
\text { Scattering }\end{array}$ & Calc. & Quar. & $\mathrm{K}-\mathrm{Fe}$ & Plag. & Kaol. & Mica & Chlor. & Mont. & Pyrite & Barite & Side & Clin. \\
\hline 11 & $384-393$ & 2 & 23 & 87.7 & 63.8 & 79.9 & 7.6 & 6.8 & 5.7 & 0.0 & 0.0 & 0.0 & 0.0 & 0.0 & 0.0 & 0.0 & 0.0 \\
\hline 11 & & & 91 & 87.8 & 64.1 & 76.5 & 8.0 & 8.4 & 7.2 & 0.0 & 0.0 & 0.0 & 0.0 & 0.0 & 0.0 & 0.0 & 0.0 \\
\hline 12 & $393-402$ & 1 & $80-82$ & 0.0 & 0.0 & 0.0 & 0.0 & 0.0 & 0.0 & 0.0 & 0.0 & 0.0 & 0.0 & 0.0 & 0.0 & 0.0 & 0.0 \\
\hline 12 & & 2 & $98-100$ & 0.0 & 0.0 & 0.0 & 0.0 & 0.0 & 0.0 & 0.0 & 0.0 & 0.0 & 0.0 & 0.0 & 0.0 & 0.0 & 0.0 \\
\hline 12 & & 3 & $109-111$ & 0.0 & 0.0 & 0.0 & 0.0 & 0.0 & 0.0 & 0.0 & 0.0 & 0.0 & 0.0 & 00 & 0.0 & 0.0 & 0.0 \\
\hline 12 & & 4 & $84-86$ & 0.0 & 0.0 & 0.0 & 0.0 & 0.0 & 0.0 & 0.0 & 0.0 & 0.0 & 0.0 & 0.0 & 0.0 & 0.0 & 0.0 \\
\hline 12 & & 5 & $5-7$ & 88.0 & 64.7 & 65.4 & 7.8 & 8.8 & 6.4 & 0.0 & 6.4 & 5.2 & 0.0 & 0.0 & 0.0 & 0.0 & 0.0 \\
\hline 13 & $402-407$ & 1 & 40 & 87.9 & 64.4 & 66.8 & 6.0 & 7.2 & 5.4 & 4.8 & 9.8 & 0.0 & 0.0 & 0.0 & 0.0 & 0.0 & 0.0 \\
\hline 13 & & 2 & 12 & 88.2 & 65.3 & 77.8 & 9.4 & 7.8 & 4.5 & 0.0 & 0.0 & 0.0 & 0.0 & 0.0 & 0.0 & 0.0 & 0.0 \\
\hline 13 & & 3 & 7 & 87.6 & 63.5 & 70.5 & 4.5 & 5.2 & 4.7 & 0.0 & 9.2 & 0.0 & 0.0 & 0.0 & 0.0 & 0.0 & 6.0 \\
\hline 14 & $407-412$ & 1 & 144 & 87.6 & 63.5 & 86.5 & 5.5 & 3.4 & 3.3 & 0.0 & 0.0 & 0.0 & 0.0 & 0.0 & 0.0 & 0.0 & 0.0 \\
\hline 15 & $412-421$ & 1 & 136 & 87.5 & 63.2 & 76.5 & 4.0 & 7.5 & 4.8 & 0.0 & 0.0 & 0.0 & 0.0 & 0.0 & 0.0 & 0.0 & 7.3 \\
\hline 15 & & 2 & 10 & 87.7 & 63.8 & 74.4 & 4.8 & 6.9 & 5.8 & 0.0 & 0.0 & 0.0 & 0.0 & 0.0 & 0.0 & 0.0 & 8.0 \\
\hline 15 & & 3 & 10 & 87.4 & 62.9 & 75.7 & 3.3 & 9.5 & 7.0 & 0.0 & 0.0 & 0.0 & 0.0 & 0.0 & 0.0 & 0.0 & 4.4 \\
\hline 15 & & 4 & 8 & 87.3 & 62.9 & 79.7 & 3.1 & 7.6 & 5.5 & 0.0 & 0.0 & 0.0 & 0.0 & 0.0 & 0.0 & 0.0 & 4.1 \\
\hline 15 & & 5 & 7 & 86.6 & 60.6 & 83.6 & 2.0 & 5.0 & 3.5 & 0.0 & 0.0 & 0.0 & 0.0 & 0.0 & 0.0 & 0.0 & 5.9 \\
\hline 16 & $421-430$ & 1 & 137 & 86.2 & 59.4 & 93.7 & 0.0 & 0.0 & 0.0 & 0.0 & 0.0 & 0.0 & 0.0 & 0.0 & 0.0 & 0.0 & 6.3 \\
\hline \multicolumn{18}{|l|}{ Hole 31} \\
\hline Core & $\begin{array}{l}\text { Depth Below } \\
\text { Sea Floor } \\
\text { (m) }\end{array}$ & Section & $\begin{array}{l}\text { Depth In } \\
\text { Section } \\
(\mathrm{cm})\end{array}$ & Diff. & $\begin{array}{c}\% \\
\text { Amorph. } \\
\text { Scattering }\end{array}$ & Calc. & Quar. & Plag. & Kaol. & Mica & Chlor. & Mont. & Clin. & & & & \\
\hline 1 & $0-9$ & 1 & 35 & 87.6 & 63.5 & 78.7 & 15.9 & 1.2 & 4.3 & 0.0 & 0.0 & 0.0 & 0.0 & & & & \\
\hline 1 & & & 90 & 87.7 & 63.8 & 77.6 & 13.8 & 1.1 & 7.5 & 0.0 & 0.0 & 0.0 & 0.0 & & & & \\
\hline 1 & & 2 & 20 & 87.7 & 63.8 & 74.2 & 16.1 & 1.5 & 8.2 & 0.0 & 0.0 & 0.0 & 0.0 & & & & \\
\hline 1 & & & 70 & 88.1 & 65.0 & 66.0 & 13.8 & 2.5 & 9.9 & 7.8 & 0.0 & 0.0 & 0.0 & & & & \\
\hline 1 & & 3 & 30 & 87.8 & 64.1 & 73.5 & 13.9 & 2.5 & 10.0 & 0.0 & 0.0 & 0.0 & 0.0 & & & & \\
\hline
\end{tabular}


TABLE 1 - Continued

\begin{tabular}{|c|c|c|c|c|c|c|c|c|c|c|c|c|c|}
\hline \multicolumn{14}{|c|}{ Hole 31 - Continued } \\
\hline Core & $\begin{array}{l}\text { Depth Below } \\
\text { Sea Floor } \\
\text { (m) }\end{array}$ & Section & $\begin{array}{l}\text { Depth In } \\
\text { Section } \\
(\mathrm{cm})\end{array}$ & Diff. & $\begin{array}{c}\% \\
\text { A morph. } \\
\text { Scattering }\end{array}$ & Calc. & Quar. & Plag. & Kaol. & Mica & Chlor. & Mont. & Clin. \\
\hline 1 & & 4 & 25 & 87.7 & 63.8 & 67.4 & 12.8 & 2.7 & 8.7 & 8.4 & 0.0 & 0.0 & 0.0 \\
\hline 1 & & & 80 & 87.4 & 62.9 & 66.8 & 9.4 & 2.0 & 0.0 & 0.0 & 5.0 & 16.8 & 0.0 \\
\hline 1 & & 5 & 18 & 87.8 & 64.1 & 79.1 & 13.1 & 1.3 & 6.5 & 0.0 & 0.0 & 0.0 & 0.0 \\
\hline 1 & & & 68 & 87.6 & 63.5 & 81.3 & 12.4 & 0.0 & 6.3 & 0.0 & 0.0 & 0.0 & 0.0 \\
\hline 1 & & 6 & $99-101$ & 0.0 & 0.0 & 0.0 & 0.0 & 0.0 & 0.0 & 0.0 & 0.0 & 0.0 & 0.0 \\
\hline 3 & $60-69$ & 1 & 21 & 87.9 & 64.4 & 79.0 & 10.7 & 0.0 & 9.8 & 0.0 & 0.0 & 0.0 & 0.0 \\
\hline 3 & & & 83 & 90.4 & 71.8 & 67.7 & 10.7 & 0.0 & 12.8 & 8.8 & 0.0 & 0.0 & 0.0 \\
\hline 3 & & 2 & 21 & 87.7 & 63.8 & 62.5 & 9.9 & 0.0 & 2.8 & 8.9 & 3.6 & 12.3 & 0.0 \\
\hline 3 & & & 71 & 87.7 & 63.8 & 75.1 & 13.8 & 0.0 & 11.1 & 0.0 & 0.0 & 0.0 & 0.0 \\
\hline 3 & & 3 & 30 & 87.4 & 62.9 & 80.8 & 11.2 & 0.0 & 8.0 & 0.0 & 0.0 & 0.0 & 0.0 \\
\hline 3 & & & 76 & 87.6 & 63.5 & 76.6 & 10.2 & 0.0 & 11.3 & 0.0 & 0.0 & 0.0 & 0.0 \\
\hline 3 & & 5 & 20 & 87.5 & 63.2 & 75.5 & 11.6 & 1.3 & 11.6 & 0.0 & 0.0 & 0.0 & 0.0 \\
\hline 3 & & & 73 & 87.7 & 63.8 & 77.0 & 12.4 & 0.0 & 10.6 & 0.0 & 0.0 & 0.0 & 0.0 \\
\hline 3 & & 6 & 21 & 87.6 & 63.5 & 80.0 & 11.1 & 0.0 & 8.9 & 0.0 & 0.0 & 0.0 & 0.0 \\
\hline 3 & & & 81 & 87.5 & 63.2 & 79.5 & 11.6 & 0.0 & 8.9 & 0.0 & 0.0 & 0.0 & 0.0 \\
\hline 4 & $69-78$ & 1 & 31 & 87.6 & 63.5 & 75.4 & 13.1 & 1.7 & 9.8 & 0.0 & 0.0 & 0.0 & 0.0 \\
\hline 4 & & 2 & 33 & 87.7 & 63.8 & 70.1 & 13.3 & 1.2 & 7.5 & 7.9 & 0.0 & 0.0 & 0.0 \\
\hline 4 & & & 92 & 87.7 & 63.8 & 75.6 & 14.2 & 0.0 & 9.2 & 0.0 & 0.0 & 0.0 & 0.0 \\
\hline 5 & $78-88$ & 1 & 40 & 87.3 & 62.6 & 79.4 & 12.3 & 1.1 & 7.3 & 0.0 & 0.0 & 0.0 & 0.0 \\
\hline 5 & & & 100 & 0.0 & 0.0 & 0.0 & 0.0 & 0.0 & 0.0 & 0.0 & 0.0 & 0.0 & 0.0 \\
\hline 6 & $88-97$ & 1 & 27 & 88.0 & 64.7 & 72.0 & 9.7 & 0.0 & 1.9 & 11.2 & 5.2 & 0.0 & 0.0 \\
\hline 6 & & & 90 & 87.7 & 63.8 & 81.5 & 10.6 & 0.0 & 7.9 & 0.0 & 0.0 & 0.0 & 0.0 \\
\hline 6 & & 2 & 12 & 87.2 & 62.4 & 78.9 & 11.3 & 0.0 & 9.9 & 0.0 & 0.0 & 0.0 & 0.0 \\
\hline 6 & & & 90 & 87.7 & 63.8 & 75.1 & 10.6 & 0.0 & 0.0 & 8.7 & 5.6 & 0.0 & 0.0 \\
\hline 7 & $97-106$ & 1 & 55 & 87.7 & 63.8 & 66.6 & 11.0 & 0.0 & 5.3 & 12.7 & 4.4 & 0.0 & 0.0 \\
\hline 7 & & & 125 & 88.9 & 67.4 & 36.1 & 24.0 & 0.0 & 12.5 & 19.7 & 7.7 & 0.0 & 0.0 \\
\hline
\end{tabular}


TABLE 1 - Continued

\begin{tabular}{|c|c|c|c|c|c|c|c|c|c|c|c|c|c|}
\hline Core & $\begin{array}{l}\text { Depth Below } \\
\text { Sea Floor } \\
\text { (m) }\end{array}$ & Section & $\begin{array}{l}\text { Depth In } \\
\text { Section } \\
\text { (cm) }\end{array}$ & Diff. & $\begin{array}{c}\% \\
\text { Amorph. } \\
\text { Scattering }\end{array}$ & Calc. & Quar. & Plag. & Kaol. & Mica & Chlor. & Mont. & Clin. \\
\hline 8 & $155-164$ & 1 & 50 & 86.9 & 61.5 & 87.4 & 9.0 & 0.0 & 3.0 & 0.0 & 0.0 & 0.0 & 0.0 \\
\hline 8 & & & 100 & 88.6 & 66.5 & 58.8 & 28.3 & 0.0 & 5.0 & 0.0 & 7.3 & 0.0 & 0.0 \\
\hline 8 & & 2 & 21 & 86.8 & 61.2 & 86.6 & 6.8 & 0.0 & 6.6 & 0.0 & 0.0 & 0.0 & 0.0 \\
\hline 8 & & & 79 & 87.7 & 63.8 & 78.0 & 12.6 & 3.9 & 4.7 & 0.0 & 0.0 & 0.0 & 0.0 \\
\hline 9 & $212-221$ & 2 & 3 & 86.7 & 60.9 & 89.9 & 3.0 & 0.0 & 0.0 & 0.0 & 0.0 & 0.0 & 7.1 \\
\hline 9 & & 3 & $108-110$ & 0.0 & 0.0 & 0.0 & 0.0 & 0.0 & 0.0 & 0.0 & 0.0 & 0.0 & 0.0 \\
\hline 10 & $270-279$ & 1 & $145-146$ & 85.8 & 58.2 & 96.4 & 1.6 & 0.0 & 0.0 & 0.0 & 0.0 & 0.0 & 0.0 \\
\hline 10 & & 2 & 141 & 85.5 & 57.4 & 97.9 & 0.0 & 0.0 & 0.0 & 0.0 & 0.0 & 0.0 & 0.0 \\
\hline 10 & & 3 & $146-148$ & 86.2 & 59.4 & 97.5 & 0.0 & 0.0 & 0.0 & 0.0 & 0.0 & 0.0 & 0.0 \\
\hline 10 & & 4 & 145 & 86.3 & 59.7 & 97.3 & 1.8 & 0.0 & 0.0 & 0.0 & 0.0 & 0.0 & 0.0 \\
\hline 10 & & 5 & 145 & 85.9 & 58.5 & 98.7 & 1.1 & 0.0 & 0.0 & 0.0 & 0.0 & 0.0 & 0.0 \\
\hline
\end{tabular}

Abbreviations for X-ray mineralogy results: Diff.-diffuse; Calc.-calcite; Quar.-quartz; K-Fe-K-feldspar; Plag.-plagioclase; Kaol.-kaolinite; Mont.-montmorillonite; Chlor.-chlorite; Cris.-cristobalite; Side.-siderite; Clin.-clinoptilolite; Sepio.-sepiolite. 
TABLE 2

X-Ray Diffraction Analysis of Samples from Leg 4 (Composited 2-20 $\mu$ Fractions, Calcite Free) ${ }^{\mathrm{a}}$

\begin{tabular}{|c|c|c|c|c|c|c|c|c|c|c|c|c|c|c|}
\hline \multicolumn{15}{|l|}{ Hole 23} \\
\hline Core & $\begin{array}{l}\text { Depth } \\
\text { (m) }\end{array}$ & Diff. & $\begin{array}{c}\% \\
\text { Amorph. } \\
\text { Scattering }\end{array}$ & Dolo. & Side. & Rhodo. & Quar. & $\mathrm{K}-\mathrm{Fe}$ & Plag. & Kaol. & Mica & Mont. & Phil. & Pyrite \\
\hline 1 & $0-9$ & 91.5 & 75.0 & 5.5 & 2.5 & 1.9 & 27.5 & 4.9 & 6.6 & 28.6 & 22.5 & 0.0 & 0.0 & 0.0 \\
\hline 2 & $53-62$ & 91.1 & 73.8 & 2.0 & 0.0 & 0.0 & 37.8 & 0.0 & 4.7 & 34.0 & 21.4 & 0.0 & 0.0 & 0.0 \\
\hline 3 & $62-71$ & 92.5 & 77.9 & 7.6 & 1.9 & 0.0 & 31.4 & 6.6 & 6.9 & 27.7 & 17.9 & 0.0 & 0.0 & 0.0 \\
\hline 4 & $112-121$ & 91.2 & 74.1 & 8.3 & 4.0 & 0.0 & 14.8 & 0.0 & 0.0 & 0.0 & 9.8 & 26.4 & 36.8 & 0.0 \\
\hline 5 & $121-131$ & 92.0 & 76.5 & 4.6 & 1.5 & 0.0 & 13.4 & 3.6 & 3.8 & 12.3 & 8.8 & 47.7 & 0.0 & 4.3 \\
\hline \multicolumn{15}{|l|}{ Hole 24} \\
\hline Core & $\begin{array}{l}\text { Depth } \\
\text { (m) }\end{array}$ & Diff. & $\begin{array}{c}\% \\
\text { Amorph. } \\
\text { Scattering }\end{array}$ & Dolo. & Side. & Quar. & $\mathrm{K}-\mathrm{Fe}$ & Plag. & Kaol. & Mica & Mont. & & & \\
\hline 1 & $198-209$ & 88.4 & 65.9 & 4.4 & 1.0 & 7.7 & 1.7 & 2.8 & 12.3 & 8.7 & 61.4 & & & \\
\hline 2 & $209-216$ & 92.0 & 76.5 & 2.6 & 0.0 & 3.7 & 0.0 & 0.0 & 8.2 & 4.6 & 79.7 & & & \\
\hline 4 & $225-234$ & 89.5 & 69.1 & 1.9 & 0.0 & 10.3 & 1.4 & 1.9 & 10.7 & 9.3 & 55.0 & & & \\
\hline \multicolumn{15}{|c|}{ Hole 24A } \\
\hline Core & $\begin{array}{c}\text { Depth } \\
\text { (m) }\end{array}$ & Diff. & $\begin{array}{c}\% \\
\text { Amorph. } \\
\text { Scattering }\end{array}$ & Quar. & Cris. & $\mathrm{K}-\mathrm{Fe}$ & Plag. & Mont. & Pyrite & Barite & & & & \\
\hline 1 & $503-511$ & 90.7 & 72.6 & 5.8 & 77.1 & 2.6 & 3.1 & 10.4 & 1.0 & 0.0 & & & & \\
\hline 2 & $511-520$ & 91.6 & 75.3 & 9.4 & 65.9 & 3.8 & 4.2 & 13.4 & 3.4 & 0.0 & & & & \\
\hline 3 & $540-550$ & 90.6 & 72.4 & 11.1 & 66.0 & 2.9 & 4.3 & 11.9 & 3.8 & 0.0 & & & & \\
\hline 4 & $550-558$ & 92.0 & 76.5 & 9.3 & 55.0 & 3.6 & 5.2 & 19.8 & 3.1 & 4.0 & & & & \\
\hline
\end{tabular}


TABLE 2 - Continued

\begin{tabular}{|c|c|c|c|c|c|c|c|c|c|}
\hline \multicolumn{10}{|l|}{ Hole 25} \\
\hline Core & $\begin{array}{l}\text { Depth } \\
\text { (m) }\end{array}$ & Diff. & $\begin{array}{c}\% \\
\text { Amorph. } \\
\text { Scattering }\end{array}$ & Dolo. & Quar. & $\mathrm{K}-\mathrm{Fe}$ & Plag. & Kaol. & Mica \\
\hline 1 & $0-9$ & 90.5 & 72.1 & 0.0 & 35.9 & 0.0 & 5.3 & 35.4 & 23.3 \\
\hline 2 & $9-18$ & 89.9 & 70.3 & 0.0 & 30.9 & 2.6 & 4.4 & 33.3 & 28.8 \\
\hline 3 & $18-27$ & 91.7 & 75.6 & 2.0 & 28.0 & 3.9 & 6.4 & 28.9 & 30.6 \\
\hline 4 & $27-36$ & 92.2 & 77.1 & 0.0 & 29.9 & 3.0 & 5.5 & 35.7 & 25.8 \\
\hline \multicolumn{10}{|c|}{ Hole $25 \mathrm{~A}$} \\
\hline Core & $\begin{array}{l}\text { Depth } \\
\text { (m) }\end{array}$ & Diff. & $\begin{array}{c}\% \\
\text { Amorph. } \\
\text { Scattering }\end{array}$ & Quar. & $\mathrm{K}-\mathrm{Fe}$ & Plag. & Kaol. & Mica & \\
\hline 1 & $49-58$ & 93.4 & 80.6 & 21.9 & 3.4 & 4.9 & 47.6 & 22.3 & \\
\hline \multicolumn{10}{|l|}{ Hole 26} \\
\hline Core & $\begin{array}{l}\text { Depth } \\
\text { (m) }\end{array}$ & Diff. & $\begin{array}{c}\% \\
\text { Amorph. } \\
\text { Scattering }\end{array}$ & Quar. & $\mathrm{K}-\mathrm{Fe}$ & Plag. & Kaol. & Mica & Chlor. \\
\hline 1 & $96-105$ & 80.7 & 43.2 & 37.1 & 0.0 & 5.9 & 9.8 & 38.5 & 6.4 \\
\hline 2 & $105-114$ & 77.9 & 35.0 & 36.7 & 0.0 & 6.3 & 9.6 & 39.4 & 6.3 \\
\hline 3 & $227-238$ & 81.2 & 44.7 & 35.4 & 2.0 & 6.2 & 13.3 & 34.6 & 5.5 \\
\hline 5 & $477-483$ & 81.3 & 45.0 & 34.9 & 2.5 & 4.8 & 11.8 & 38.3 & 5.6 \\
\hline
\end{tabular}


TABLE 2 - Continued

\begin{tabular}{|c|c|c|c|c|c|c|c|c|c|c|c|c|}
\hline \multicolumn{13}{|l|}{ Hole 27} \\
\hline Core & $\begin{array}{l}\text { Depth } \\
\text { (m) }\end{array}$ & Diff. & $\begin{array}{c}\% \\
\text { Amorph. } \\
\text { Scattering }\end{array}$ & Quar. & $\mathrm{K}-\mathrm{Fe}$ & Plag. & Kaol. & Mica & Chlor. & Mont. & Pyrite & Side. \\
\hline 1 & $84-93$ & 91.0 & 73.5 & 40.5 & 2.4 & 2.6 & 25.0 & 22.1 & 7.4 & 0.0 & 0.0 & 0.0 \\
\hline 1 & & 91.0 & 73.5 & 17.7 & 0.0 & 1.3 & 7.4 & 13.6 & 5.3 & 54.0 & 0.0 & 0.0 \\
\hline 1 & & 91.7 & 75.6 & 17.7 & 0.0 & 5.2 & 18.0 & 15.0 & 0.0 & 43.3 & 0.0 & 0.0 \\
\hline 2 & $141-150$ & 91.0 & 73.5 & 16.7 & 0.0 & 5.0 & 34.7 & 11.0 & 0.0 & 32.7 & 0.0 & 0.0 \\
\hline 2 & & 84.7 & 55.0 & 6.2 & 0.0 & 1.1 & 20.9 & 6.5 & 4.7 & 59.7 & 0.0 & 0.0 \\
\hline 3 & $235-244$ & 90.0 & 70.6 & 27.5 & 4.1 & 4.4 & 27.5 & 22.6 & 0.0 & 9.9 & 3.9 & 0.0 \\
\hline 4 & $244-249$ & 90.9 & 73.2 & 25.0 & 0.0 & 5.2 & 27.0 & 16.5 & 0.0 & 22.2 & 4.0 & 0.0 \\
\hline 5 & $369-378$ & 90.6 & 72.4 & 23.3 & 3.5 & 3.3 & 22.3 & 13.3 & 8.6 & 12.3 & 3.3 & 10.2 \\
\hline 6 & $454-463$ & 90.4 & 71.8 & 14.7 & 0.0 & 1.8 & 11.5 & 8.4 & 5.4 & 58.3 & 0.0 & 0.0 \\
\hline
\end{tabular}

Hole 27A

\begin{tabular}{cccccccccccc}
\hline & $\begin{array}{c}\text { \% } \\
\text { Core } \\
(\mathrm{m})\end{array}$ & Diff. & $\begin{array}{c}\text { Amorph. } \\
\text { Scattering }\end{array}$ & Dolo. & Quar. & K-Fe & Plag. & Kaol. & Mica & Chlor. & Mont. \\
\hline 1 & $25-34$ & 90.5 & 72.1 & 0.0 & 43.7 & 0.0 & 6.3 & 6.2 & 31.5 & 12.4 & 0.0 \\
2 & $45-54$ & 89.9 & 70.3 & 0.0 & 34.4 & 2.2 & 5.7 & 24.4 & 33.4 & 0.0 & 0.0 \\
3 & $54-63$ & 87.9 & 64.4 & 0.0 & 39.0 & 3.3 & 7.6 & 12.6 & 32.4 & 5.1 & 0.0 \\
4 & $63-72$ & 88.4 & 65.9 & 0.0 & 35.6 & 0.0 & 6.5 & 22.1 & 35.7 & 0.0 & 0.0 \\
5 & $72-81$ & 90.8 & 72.9 & 0.0 & 24.1 & 3.9 & 4.3 & 7.7 & 23.6 & 6.3 & 30.1 \\
\hline
\end{tabular}


TABLE 2 - Continued

\begin{tabular}{|c|c|c|c|c|c|c|c|c|c|c|c|c|c|}
\hline \multicolumn{14}{|l|}{ Hole 28} \\
\hline Core & $\begin{array}{l}\text { Depth } \\
\text { (m) }\end{array}$ & Diff. & $\begin{array}{c}\% \\
\text { Amorph. } \\
\text { Scattering }\end{array}$ & Side. & Quar. & Cris. & $\mathrm{K} \cdot \mathrm{Fe}$ & Plag. & Kaol. & Mica & Chlor. & Mont. & Clin. \\
\hline 2 & $68-77$ & 92.3 & 77.1 & 0.0 & 21.0 & 0.0 & 0.0 & 9.3 & 18.5 & 13.9 & 0.0 & 33.7 & 3.6 \\
\hline 2 & & 93.3 & 80.3 & 0.0 & 25.7 & 0.0 & 0.0 & 7.7 & 19.5 & 10.5 & 0.0 & 36.5 & 0.0 \\
\hline 3 & $169-176^{\mathrm{a}}$ & 89.7 & 69.7 & 0.0 & 16.9 & 9.0 & 2.4 & 2.5 & 13.5 & 8.3 & 0.0 & 39.0 & 8.4 \\
\hline 3 & & 89.5 & 69.1 & 2.5 & 13.9 & 11.8 & 1.4 & 2.9 & 3.9 & 6.8 & 10.0 & 34.6 & 12.1 \\
\hline 3 & & 91.3 & 74.4 & 1.0 & 7.5 & 0.0 & 0.0 & 0.0 & 6.0 & 0.0 & 0.0 & 77.1 & 4.5 \\
\hline \multicolumn{14}{|c|}{${ }^{a}$ Plot indicates 9 meters of recovery in order to accomodate the samples received. } \\
\hline \multicolumn{14}{|l|}{ Hole 29} \\
\hline Core & $\begin{array}{l}\text { Depth } \\
\text { (m) }\end{array}$ & Diff. & $\begin{array}{c}\% \\
\text { Amorph. } \\
\text { Scattering }\end{array}$ & Quar. & $\mathrm{K}-\mathrm{Fe}$ & Plag. & Kaol. & Mica & Mont. & Clin. & & & \\
\hline 1 & $0-9$ & 89.0 & 67.6 & 37.2 & 1.9 & 3.0 & 32.1 & 25.9 & 0.0 & 0.0 & & & \\
\hline 2 & 9.18 & 88.4 & 65.9 & 33.3 & 2.8 & 2.7 & 34.8 & 26.4 & 0.0 & 0.0 & & & \\
\hline 3 & $18-27$ & 90.7 & 72.6 & 38.5 & 1.9 & 4.0 & 35.0 & 20.5 & 0.0 & 0.0 & & & \\
\hline 4 & $27-37$ & 90.6 & 72.4 & 42.5 & 0.0 & 4.8 & 30.3 & 22.4 & 0.0 & 0.0 & & & \\
\hline 5 & $37-46$ & 90.2 & 71.2 & 40.8 & 3.7 & 6.8 & 27.3 & 31.4 & 0.0 & 0.0 & & & \\
\hline 6 & $46-55$ & 90.6 & 72.4 & 41.9 & 2.2 & 3.7 & 29.3 & 21.6 & 0.0 & 0.0 & & & \\
\hline 7 & $117-126$ & 91.0 & 73.5 & 11.2 & 5.8 & 8.8 & 16.6 & 0.0 & 20.0 & 37.5 & & & \\
\hline 9 & $135-144$ & 98.3 & 95.0 & 53.2 & 5.3 & 11.7 & 29.8 & 0.0 & 0.0 & 0.0 & & & \\
\hline 10 & $144-153$ & 99.1 & 97.4 & 61.3 & 11.0 & 27.6 & 0.0 & 0.0 & 0.0 & 0.0 & & & \\
\hline 11 & $153-162$ & 99.0 & 97.1 & 66.2 & 15.9 & 17.9 & 0.0 & 0.0 & 0.0 & 0.0 & & & \\
\hline 12 & $162-172$ & 99.1 & 97.4 & 34.0 & 5.1 & 11.2 & 21.8 & 27.9 & 0.0 & 0.0 & & & \\
\hline
\end{tabular}


TABLE 2 - Continued

\begin{tabular}{|c|c|c|c|c|c|c|c|c|c|c|}
\hline \multicolumn{11}{|c|}{ Hole 29 - Continued } \\
\hline Core & $\begin{array}{l}\text { Depth } \\
\text { (m) }\end{array}$ & Diff. & $\begin{array}{c}\% \\
\text { Amorph. } \\
\text { Scattering }\end{array}$ & Quar. & $\mathrm{K}-\mathrm{Fe}$ & Plag. & Kaol. & Mica & Mont. & Clin. \\
\hline 14 & $184-193$ & 96.9 & 90.9 & 40.0 & 0.0 & 6.4 & 20.8 & 32.8 & 0.0 & 0.0 \\
\hline 15 & $193-202$ & 99.0 & 97.1 & 50.8 & 22.8 & 26.4 & 0.0 & 0.0 & 0.0 & 0.0 \\
\hline 16 & $202-211$ & 99.3 & 97.9 & 7.7 & 0.0 & 3.5 & 7.7 & 12.6 & 68.5 & 0.0 \\
\hline 17 & $211-220$ & 98.8 & 96.5 & 60.2 & 0.0 & 39.8 & 0.0 & 0.0 & 0.0 & 0.0 \\
\hline 18 & $220-225$ & 98.7 & 96.2 & 3.5 & 0.0 & 1.8 & 0.0 & 0.0 & 94.6 & 0.0 \\
\hline \multicolumn{11}{|c|}{ Hole 29A } \\
\hline Core & $\begin{array}{l}\text { Depth } \\
\text { (m) }\end{array}$ & Diff. & $\begin{array}{c}\% \\
\text { Amorph. } \\
\text { Scattering }\end{array}$ & Quar. & Plag. & Kaol. & Mica & Chlor. & Mont. & \\
\hline 2 & $50-59$ & 92.0 & 76.5 & 41.5 & 8.7 & 18.3 & 23.6 & 7.9 & 0.0 & \\
\hline 4 & $68-77$ & 92.0 & 76.5 & 29.8 & 8.3 & 23.8 & 17.0 & 0.0 & 21.0 & \\
\hline 5 & $77-86$ & 92.0 & 76.5 & 29.6 & 8.3 & 15.4 & 9.8 & 0.0 & 37.0 & \\
\hline \multicolumn{11}{|c|}{ Hole 29B } \\
\hline Core & $\begin{array}{l}\text { Depth } \\
\text { (m) }\end{array}$ & Diff. & $\begin{array}{c}\% \\
\text { Amorph. } \\
\text { Scattering }\end{array}$ & Quar. & Plag. & Kaol. & Mica & Chlor. & Mont. & Clin. \\
\hline 1 & $57-66$ & 90.3 & 71.5 & 30.5 & 6.4 & 8.5 & 25.0 & 7.9 & 21.6 & 0.0 \\
\hline 2 & $68-77$ & 91.5 & 75.0 & 31.7 & 8.6 & 21.6 & 15.6 & 0.0 & 22.5 & 0.0 \\
\hline 3 & $78-87$ & 92.2 & 77.1 & 31.6 & 8.2 & 19.0 & 13.0 & 0.0 & 28.2 & 0.0 \\
\hline 4 & $87-97$ & 91.8 & 75.9 & 34.7 & 7.6 & 6.9 & 17.0 & 9.0 & 24.7 & 0.0 \\
\hline 5 & $97-106$ & 92.8 & 78.8 & 45.7 & 16.4 & 27.4 & 0.0 & 0.0 & 0.0 & 10.5 \\
\hline
\end{tabular}


TABLE 2 - Continued

\begin{tabular}{|c|c|c|c|c|c|c|c|c|c|c|c|c|c|c|}
\hline \multicolumn{15}{|c|}{ Hole 29B - Continued } \\
\hline Core & $\begin{array}{c}\text { Depth } \\
\text { (m) }\end{array}$ & Diff. & $\begin{array}{c}\% \\
\text { Amorph. } \\
\text { Scattering }\end{array}$ & Quar. & Plag. & Kaol. & Mica & Chlor. & Mont. & Clin. & & & & \\
\hline 6 & $106-115$ & 94.3 & 83.2 & 37.3 & 20.1 & 23.9 & 12.3 & 0.0 & 0.0 & 6.3 & & & & \\
\hline 8 & $124-133$ & 97.6 & 92.9 & 18.7 & 5.6 & 0.0 & 0.0 & 0.0 & 66.4 & 9.3 & & & & \\
\hline 9 & $218-227$ & 98.7 & 96.2 & 4.5 & 0.0 & 0.0 & 0.0 & 0.0 & 95.1 & 0.0 & & & & \\
\hline 10 & $227-232$ & 99.8 & 99.4 & 91.7 & 8.3 & 0.0 & 0.0 & 0.0 & 0.0 & 0.0 & & & & \\
\hline \multicolumn{15}{|l|}{ Hole 30} \\
\hline Core & $\begin{array}{c}\text { Depth } \\
\text { (m) }\end{array}$ & Diff. & $\begin{array}{c}\% \\
\text { Amorph. } \\
\text { Scattering }\end{array}$ & Side. & Quar. & $\mathrm{K}-\mathrm{Fe}$ & Plag. & Kaol. & Mica & Chlor. & Mont. & Clin. & Hema & Pyrite \\
\hline 1 & $50-59$ & 90.1 & 70.9 & 0.0 & 28.2 & 0.0 & 6.8 & 20.3 & 23.2 & 0.0 & 20.1 & 0.0 & 0.0 & 1.4 \\
\hline 2 & $59-69$ & 88.6 & 66.5 & 0.0 & 42.4 & 0.0 & 5.7 & 27.6 & 21.6 & 0.0 & 0.0 & 0.0 & 0.0 & 2.8 \\
\hline 3 & $108-117$ & 88.4 & 65.9 & 0.0 & 45.7 & 0.0 & 6.0 & 18.7 & 20.6 & 5.3 & 0.0 & 0.0 & 0.0 & 3.7 \\
\hline 4 & $117-126$ & 89.3 & 68.5 & 1.0 & 37.9 & 0.0 & 6.3 & 25.2 & 25.2 & 0.0 & 0.0 & 0.0 & 0.0 & 3.2 \\
\hline 5 & $164-174$ & 89.4 & 68.8 & 2.1 & 41.9 & 0.0 & 5.0 & 29.0 & 18.0 & 0.0 & 0.0 & 0.0 & 0.0 & 2.8 \\
\hline 6 & $174-178$ & 87.5 & 63.2 & 1.4 & 32.5 & 2.9 & 4.5 & 20.8 & 18.5 & 0.0 & 17.2 & 0.0 & 0.0 & 2.3 \\
\hline 7 & $260-269$ & 87.5 & 63.2 & 1.2 & 42.4 & 0.0 & 5.2 & 19.6 & 22.5 & 5.6 & 0.0 & 0.0 & 0.0 & 3.5 \\
\hline 9 & $366-375$ & 90.4 & 71.8 & 0.0 & 17.2 & 0.0 & 27.2 & 9.0 & 18.4 & 0.0 & 21.6 & 2.9 & 3.6 & 0.0 \\
\hline 10 & $375-384$ & 91.5 & 75.0 & 0.0 & 18.9 & 0.0 & 34.0 & 12.8 & 10.5 & 0.0 & 23.6 & 0.0 & 0.0 & 0.0 \\
\hline 11 & $384-393$ & 91.4 & 74.7 & 1.3 & 18.9 & 0.0 & 16.2 & 8.3 & 15.5 & 0.0 & 33.6 & 3.2 & 0.0 & 3.0 \\
\hline 12 & $343-402$ & 91.4 & 74.7 & 0.0 & 17.6 & 0.0 & 18.3 & 1.1 & 9.6 & 4.5 & 44.0 & 0.0 & 0.0 & 4.2 \\
\hline 13 & $402-407$ & 91.0 & 73.5 & 0.0 & 20.7 & 0.0 & 15.6 & 10.0 & 10.2 & 0.0 & 33.2 & 4.8 & 0.0 & 5.6 \\
\hline
\end{tabular}


TABLE 2 - Continued

\begin{tabular}{|c|c|c|c|c|c|c|c|c|c|c|c|c|c|c|}
\hline \multicolumn{15}{|c|}{ Hole 30 - Continued } \\
\hline Core & $\begin{array}{l}\text { Depth } \\
\text { (m) }\end{array}$ & Diff. & $\begin{array}{c}\% \\
\text { Amorph. } \\
\text { Scattering }\end{array}$ & Side. & Quar. & $\mathrm{K}-\mathrm{Fe}$ & Plag. & Kaol. & Mica & Chlor. & Mont. & Clin. & Hema & Pyrite \\
\hline 14 & $407-412$ & 91.1 & 73.8 & 0.0 & 16.5 & 0.0 & 19.1 & 0.0 & 12.1 & 4.3 & 11.7 & 32.9 & 0.0 & 3.3 \\
\hline 15 & $412-421$ & 93.2 & 80.0 & 0.0 & 15.8 & 0.0 & 24.3 & 0.0 & 9.0 & 0.0 & 19.7 & 28.9 & 0.0 & 2.5 \\
\hline 16 & $421-430$ & 90.4 & 71.8 & 0.0 & 10.9 & 0.0 & 9.6 & 0.0 & 0.0 & 5.8 & 13.6 & 54.6 & 0.0 & 5.5 \\
\hline \multicolumn{15}{|l|}{ Hole 31} \\
\hline Core & $\begin{array}{l}\text { Depth } \\
\text { (m) }\end{array}$ & Diff. & $\begin{array}{c}\% \\
\text { Amorph. } \\
\text { Scattering }\end{array}$ & Quar. & $\mathrm{K}-\mathrm{Fe}$ & Plag. & Kaol. & Mica & Chlor. & Mont. & Clin. & Pyrite & & \\
\hline 1 & $0-9$ & 89.2 & 68.2 & 51.3 & 2.1 & 5.1 & 18.6 & 16.0 & 7.0 & 0.0 & 0.0 & 0.0 & & \\
\hline 3 & $60-69$ & 88.4 & 65.9 & 47.4 & 3.2 & 5.7 & 18.4 & 15.6 & 9.5 & 0.0 & 0.0 & 0.0 & & \\
\hline 4 & $69-78$ & 89.5 & 69.1 & 44.5 & 0.0 & 4.3 & 22.4 & 18.2 & 18.4 & 0.0 & 0.0 & 2.3 & & \\
\hline 5 & $78-88$ & 89.2 & 68.2 & 59.4 & 0.0 & 6.4 & 14.5 & 3.7 & 12.0 & 0.0 & 0.0 & 2.2 & & \\
\hline 6 & $88-97$ & 88.5 & 66.2 & 48.2 & 0.0 & 5.7 & 18.6 & 19.4 & 8.1 & 0.0 & 0.0 & 0.0 & & \\
\hline 7 & $97-106$ & 88.9 & 67.4 & 48.8 & 0.0 & 4.1 & 18.3 & 19.1 & 5.8 & 0.0 & 0.0 & 2.3 & & \\
\hline 8 & $155-164$ & 90.6 & 72.4 & 40.6 & 4.1 & 4.1 & 13.0 & 10.2 & 6.5 & 21.5 & 0.0 & 0.0 & & \\
\hline 9 & $212-221$ & 90.0 & 70.6 & 24.9 & 0.0 & 12.7 & 0.0 & 0.0 & 0.0 & 0.0 & 62.3 & 0.0 & & \\
\hline 10 & $270-279$ & 93.3 & 80.3 & 46.0 & 0.0 & 16.2 & 0.0 & 0.0 & 37.8 & 0.0 & 0.0 & 0.0 & & \\
\hline
\end{tabular}

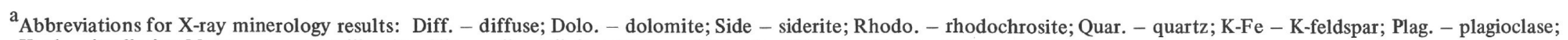
Kaol. - kaolimite; Mont. - montmorillomite; Clin. - clinoptilolete; Phil. - phillipsite; Chlor. - chlorite; Cris. - cristobalite. 